\title{
A smartphone intervention that enhances real-world memory and promotes differentiation of hippocampal activity in older adults
}

Chris B. Martin ${ }^{1}$, Bryan Hong ${ }^{2}$, Rachel N. Newsome ${ }^{2}$, Katarina Savel ${ }^{2}$, Melissa E. Meade ${ }^{2}$, Andrew Xia ${ }^{2}$, Christopher J. Honey ${ }^{3}$, Morgan D. Barense ${ }^{2,4}$

${ }^{1}$ Department of Psychology, Florida State University, Tallahassee, FL, 32306 USA

2 Department of Psychology, University of Toronto, Toronto, ON, M5S 3G3 Canada

${ }^{3}$ Department of Psychological \& Brain Sciences, Johns Hopkins University, Baltimore, MD 21218, USA

${ }^{4}$ Rotman Research Institute, Baycrest Hospital, Toronto, ON, M6A 2X8 Canada

Corresponding authors:

Chris B. Martin

Department of Psychology

1107 West Call Street,

Tallahassee, Florida 32306

Phone: 850-645-0654

E-Mail: cmartin@psy.fsu.edu
Morgan D. Barense

Department of Psychology

100 St. George Street,

Toronto, Ontario M5S $3 \mathrm{G} 3$

Phone: 416-978-5429

E-Mail: morgan.barense@utoronto.ca

Keywords: autobiographical memory, episodic memory, hippocampus, aging, smartphone intervention, fMRI 


\section{Abstract}

The act of remembering an everyday experience influences how we interpret the world, how we think about the future, and how we perceive ourselves. It also enhances longterm retention of the recalled content, increasing the likelihood that it will be recalled again. Unfortunately, the ability to recollect event-specific details and re-experience the past tends to decline with age. This decline in recollection may reflect a corresponding decrease in the distinctiveness of hippocampal memory representations. Despite these well-established changes, there are few effective cognitive behavioral interventions that target real-world episodic memory. We addressed this gap by developing a smartphonebased application called HippoCamera that allows participants to record labelled videos of everyday events and subsequently replay, high-fidelity autobiographical memory cues. In two experiments, we found that older adults were able to easily integrate this noninvasive intervention into their daily lives. Using HippoCamera to repeatedly reactivate memories for real-world events improved episodic recollection and it evoked more positive autobiographical sentiment at the time of retrieval. In both experiments, these benefits were observed shortly after the intervention and again after a 3-month delay. Moreover, more detailed recollection was associated with more differentiated memory signals in the hippocampus. Thus, using this smartphone application to systematically reactivate memories for recent real-world experiences can help to maintain a bridge between the present and past in older adults.

\section{Significance Statement}

The ability to vividly recollect our past declines with age, a trend that negatively impacts overall well-being. We show that using smartphone technologies to record and replay brief but rich memory cues from daily life can improve older adults' ability to re-experience the past. This enhancement was associated with corresponding changes in the way memories were stored in the brain. Functional neuroimaging showed that repeatedly replaying memory cues drove memories apart from one another in the hippocampus, a brain region with well-established links to memory function. This increase in differentiation likely facilitated behavior by strengthening memory and minimizing competition among different memories at retrieval. This work reveals a novel, easy-to-use intervention that helps older adults better remember their personal past. 


\section{Introduction}

Autobiographical memory enables us to remember our personal past, and by extension contributes to our sense of identity $(1,2)$, the maintenance of social relationships $(3,4)$, and the ability to think about a self-relevant future (5-7). Retrieving an autobiographical memory is a layered process that involves recovery of general semantic knowledge (e.g., knowing what typically happens at a youth baseball game), personal semantic knowledge (e.g., knowing that you have a grandson who plays baseball), and recollection of episodic details that were unique to a specific event (e.g., remembering the look on your grandson's face the first time he hit the ball). In neurologically healthy individuals, the ability to retrieve general and personal semantic knowledge typically remains constant across the lifespan, whereas recollection of event-specific details tends to decline with age, compromising our ability to vividly remember the past (8). The trajectory of this decline has been linked to corresponding reductions in the structural and functional integrity of the hippocampus $(9,10)$, which supports the encoding and retrieval of event-specific details from recent experiences $(11,12)$. Although memory decline is common in aging and significantly worsens quality of life, very few interventions specifically target autobiographical episodic memory. To fill this gap, we developed a smartphone application called HippoCamera, which is inspired by hippocampal function and designed to improve episodic recollection of real-world events in older adults.

HippoCamera is a digital memory aid that embodies principles from cognitive psychology and neuroscience known to improve memory, including distributed learning (13), deep encoding (14), the use of self-generated cues (15), the use of multi-modal cues (16), and strengthening contextual associations for events (17). It provides users a tool to record and replay personalized, high-fidelity cues that capture the complex and dynamic nature of the real-world events they value most (Fig. 1A-B). A HippoCamera cue consists of a self-generated 8-second verbal description (e.g., "Felix is playing baseball at Tom Brown Park") that is played concurrently with an 8-second speeded video (i.e., a 24-second video played at 3x speed, a design choice that enables efficient review and was inspired by the temporally compressed nature of endogenous hippocampal replay) (18). The application automatically curates replay sessions consisting of up to five distinct cues selected to balance distributed learning and prioritization of recent significant events 
over remote insignificant events. In the current study, cues were assigned to either a Replay condition and viewed multiple times, or a within-subjects Baseline condition and never replayed. This design allowed us to target and characterize the effect of replaying HippoCamera cues while holding all other factors constant.

Hippocamera draws on the well-established phenomenon that a memory can be strengthened by retrieval, which alters how it is represented in the hippocampus $(19,20)$ and increases the probability of successfully retrieving it in the future (21). Evidence from computational modeling $(22,23)$, behavioral investigations $(24,25)$, and neuroimaging research (26-28) suggests that strengthening is particularly likely when retrieval cues evoke strong neural reactivation of an episodic memory trace. Retrieval-related strengthening has variably been linked to hippocampal integration (i.e., increased neural similarity among memories; 29-35) and hippocampal differentiation (i.e., decreased neural similarity among memories; $36-40$ ); both forms of representational change can reduce competition at retrieval (23). Importantly, integration tends to be observed when recall involves recovery of episodic gist, whereas differentiation is most apparent when engaging in elaborative recall of event-specific details (41). To the extent that repeatedly replaying high-fidelity HippoCamera cues evokes strong memory reactivation and elaborative recall, we hypothesized that its use would strengthen episodic autobiographical memory by promoting hippocampal differentiation, which would enable more detailed recall of event-specific information.

Memory strengthening may also be reflected in improved autobiographical sentiment. Mood disorders, including depression, are associated with impaired episodic memory for personally experienced events with intact semantic memory for general information and facts (42). Impairments in episodic memory negatively impact quality of life by reducing one's sense of identity, confidence, and autonomy (43), and therefore further degrade emotional well-being. Moreover, episodic memory deficits are compounded in seniors with depression (44), highlighting the need for interventions that target episodic memory. As such, we also tested the hypothesis that replaying HippoCamera cues encourages repeated positive reminiscence and that this would be reflected in improved autobiographical sentiment. 
Across two experiments, we found that older adults were able to easily incorporate HippoCamera into their daily lives with little supervision. Doing so enhanced episodic recollection of everyday experiences and produced more positive autobiographical sentiment at retrieval. Using fMRI, we revealed that replaying memory cues increased differentiation of activity patterns in the hippocampus, a measure that was positively correlated with the amount of event-specific episodic information that could be recalled. These findings indicate that repeated replay promoted differentiation of autobiographical memory representations in the hippocampus in a manner that facilitated detail-rich episodic retrieval.

\section{Results}

\section{Cued Reactivation Improved Recollection of Event-Specific Detail}

Experiment 1. Participants $(N=22$, mean age 69.64 years \pm 0.89 SEM, 16 women) used HippoCamera to record and replay episodic memory cues for events that took place over two consecutive weeks (Fig. 1C). During this time, they were instructed to record 5 events per day and view 6 replay sessions per day. Moreover, they were encouraged to distribute their replay sessions throughout each day, rather than view them in succession. This guidance was reinforced by smartphone notifications that intermittently reminded participants to record and replay. Compliance was generally high with an average of $4.8 \pm 0.20$ SEM cues recorded per day and $5.4 \pm 0.27$ SEM replay sessions viewed per day. Cues were randomly assigned to either the Replayed or Baseline condition on a per cue basis, meaning Replayed and Baseline events were interleaved both within and across days. Cues in the Replayed condition were viewed an average of $8.7 \pm 0.42$ SEM times prior to memory testing. Cues in the Baseline condition were never replayed and provided a within-subject comparison. 
A Recording Autobiographical Memory Cues

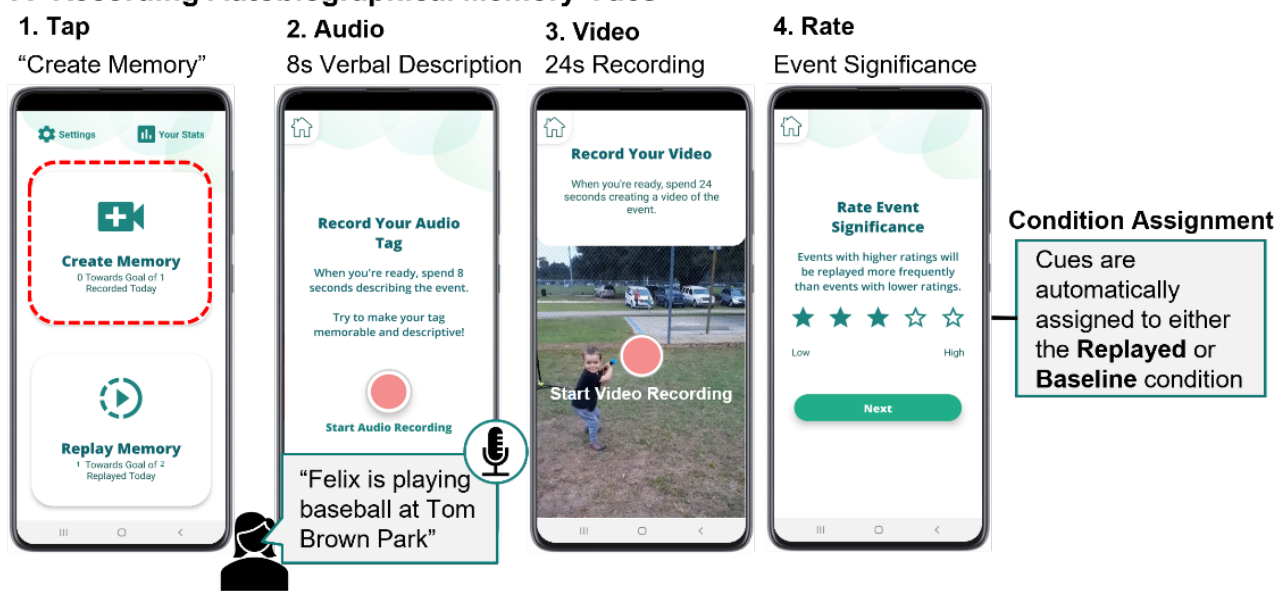

B Replaying Autobiographical Memory Cues

1. Tap 2. Replay (up to 5 cues)

"Replay Memory" Verbal descriptions played with $3 x$ speeded videos

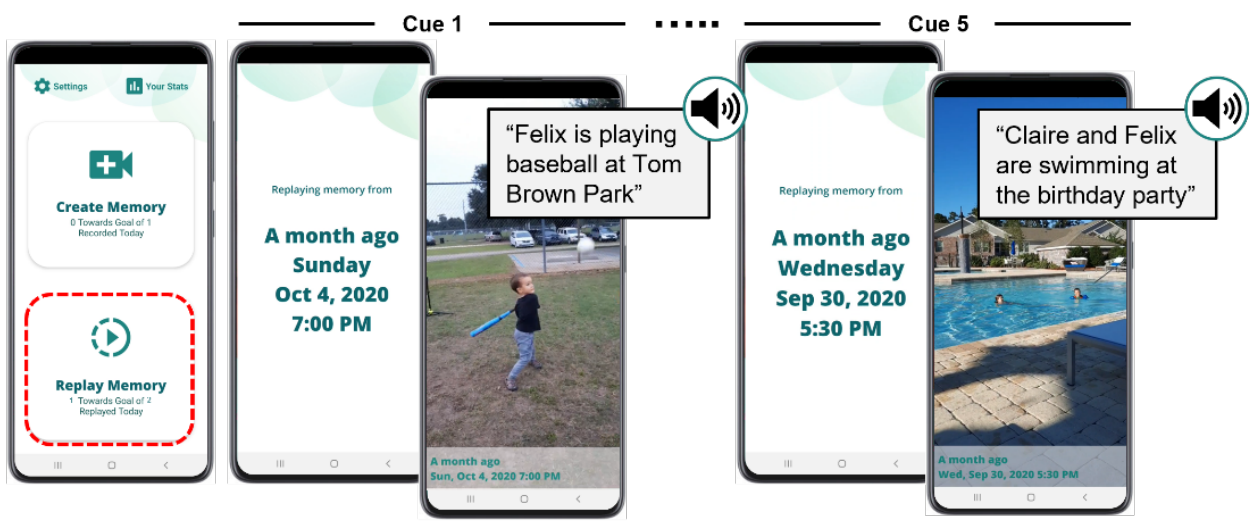

C Experiment 1: 2-Week Intervention

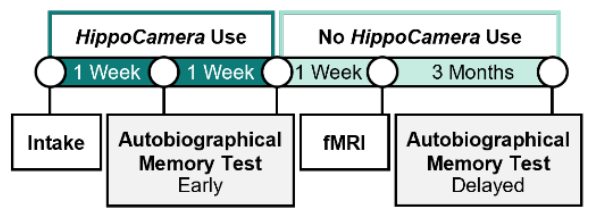

D Experiment 2: 10-Week Intervention

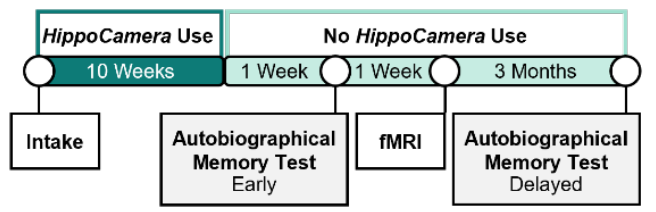

Fig. 1. HippoCamera application and experimental timelines. (A) Cues are recorded in four steps: (1) Select a real-world event and initiate recording, (2) record an 8-second verbal description, (3) record a 24-second video, and (4) rate the event's significance. (B) Replay sessions consist of up to 5 cues played sequentially. During replay, the 8 -second audio is played concurrently with a speeded (3x) version of the video. Each cue is preceded by a text display that indicates approximately how much time has passed since the event as well as its exact date and time. (C) Experiment 1 timeline. (D) Experiment 2 timeline.

We assessed autobiographical memory performance using a cued-recall test administered at multiple time points - Early and Delayed (Fig. 1C). Early testing was completed immediately after the first and second week of HippoCamera use, with each 
assessment targeting memories for a subset of events that were recorded over the previous seven-day period. There were no significant differences in the number of details recalled between the first and second week of early testing $(F(1,21)=0.72, P=0.406)$, nor was there an interaction between week and replay condition $(F(1,21)=0.11, P=$ 0.744). Delayed testing was completed 3.25 months after Early testing. Particidpants did not have access to their memory cues during this interval. We tested memory for the same set of events at Early testing, Delayed testing, and in the subsequent fMRI experiment (mean number of Replayed trials $=17.1 \pm 0.61$ SEM, Baseline trials $=17.2 \pm$ 0.61 SEM). We selected cues for the purpose of testing in a manner that optimized matching between Replayed and Baseline memories in event significance, event frequency, and memory age (SI Appendix 1.1.3).

On each trial of our autobiographical memory tests, participants first viewed one of their 8-second self-generated cues, composed of the video and its associated verbal description. In all cases, this would be either a cue that had been recorded and then repeatedly replayed with HippoCamera (i.e., the Replayed condition) or a cue that had been recorded but not previously replayed with HippoCamera (i.e., the Baseline condition). Participants subsequently verbally recalled their memory for the corresponding event. They were encouraged to provide as many details as possible about the target event and were provided with an example response at the beginning of each test session (SI Appendix 1.1.1). Responses were scored to quantify retrieval of Internal, External, and In-Cue details (8, 45; SI Appendix 1.1.2). Internal details are event-specific and reflect episodic re-experiencing (e.g., recollecting the look on your grandson's face when the first time he hit a baseball). External details reflect retrieval of general semantic information (e.g., knowing what tends to happen at a baseball game), personal semantics (e.g., knowing that your grandson is 4 years old), or details from a non-target event (e.g., recollecting what happened in a different baseball game in which your grandson played). In-Cue details reflect descriptions of information captured by the cues themselves, which may or may not involve memory retrieval. As such, In-Cue details did not contribute to either Internal or External detail counts $(46,47)$. 
Internal detail counts are shown in Fig. $2 A$. In line with our hypothesis, a Poisson generalized linear mixed model revealed a significant main effect of condition (Replayed vs. Baseline: $b=0.212, S E=0.0183, z=11.593, P<.001$; SI Appendix 1.2, Table S1), such that events in the Replayed condition were recalled with significantly more Internal details than were those in the Baseline condition. Proportionally, this reflected a $55.8 \%$ increase in Internal details for Replayed relative to Baseline trials at Early testing. Importantly, the benefit of replay on recall of Internal details was observed in every participant. In addition to a main effect of condition, we found a main effect of test time point (Early vs. Delayed: $b=-0.292$, SE $=0.0336, z=-8.676, P<.001$ ), indicating that participants recalled significantly more Internal details at Early testing than they did at Delayed testing. Although the overall number of Internal details declined over time, the relative benefit of cued reactivation was preserved: Replayed events were recalled with $58.9 \%$ more event-specific details than Baseline events at Delayed testing. This pattern of results was also obtained after adjusting for verbal output by dividing detail count by word count on a trial-by-trial basis (SI Appendix 2.5, Table S5) (48, 49). Moreover, repeated HippoCamera replay benefitted all subtypes of Internal detail (Internal Event, Time, Place, Perceptual, and Thought/Emotion; SI Appendix 2.3, Fig. S2, Table S2-3). Together, these data suggest that replay initially enhanced episodic autobiographical memory and that these enhancements persisted three months after discontinuation of HippoCamera use.

We next examined whether repeatedly replaying HippoCamera memory cues influenced retrieval of External details. This set of exploratory analyses revealed a significant main effect of condition ( $b=-0.0335$, SE $=0.0171, z=-1.964, p=.0495)$ and a significant interaction between condition and test time point $(b=0.0342$, SE $=0.0172$, $z=1.986, p=.0471)$. These effects reflect the fact that participants recalled significantly fewer External details for Replayed as compared to Baseline events at Early testing $(b=$ $-0.135, \mathrm{SE}=0.0520, z=-2.603, \mathrm{P}=.0092)$ but not Delayed testing $(b=0.00139, \mathrm{SE}=$ $0.0447, z=0.031, \mathrm{P}=.975 ; \mathrm{SI}$ Appendix 2.1, SI Appendix 2.4, Fig. S1, Fig. S3, Table S1, Table S3-4). Overall, these data indicate that using HippoCamera selectively enhanced recall of event-specific episodic information. 
Lastly, we analysed the number of In-Cue details to determine whether HippoCamera replay made participants more reliant on cues at retrieval. We found a main effect of condition ( $b=-0.207$, SE $=0.0295, z=-7.024, p<.001)$ that reflected fewer InCue details in the Replayed condition than the Baseline condition. This result indicates that participants relied less on information reflected in their cues and more on elaborative retrieval processes following repeated replay with HippoCamera (SI Appendix 2.2, Fig. S1, Table S1).

A

35.00

30.00

25.00

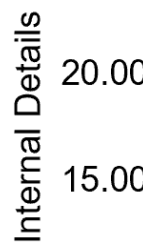

10.00

5.00

0.00

.

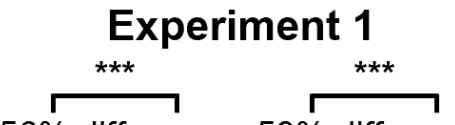

$56 \%$ difference $59 \%$ difference

35.00
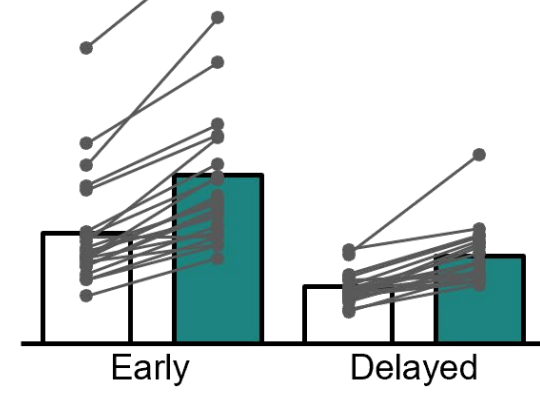

Baseline

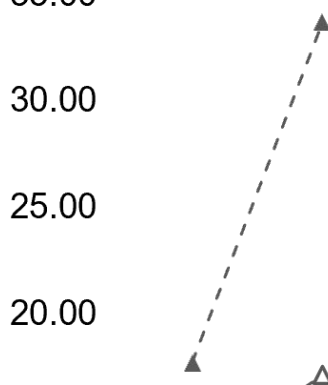

15.00

B Experiment 2

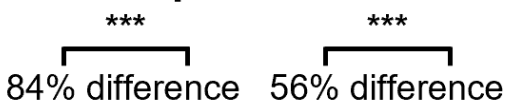

$84 \%$ difference $56 \%$ difference

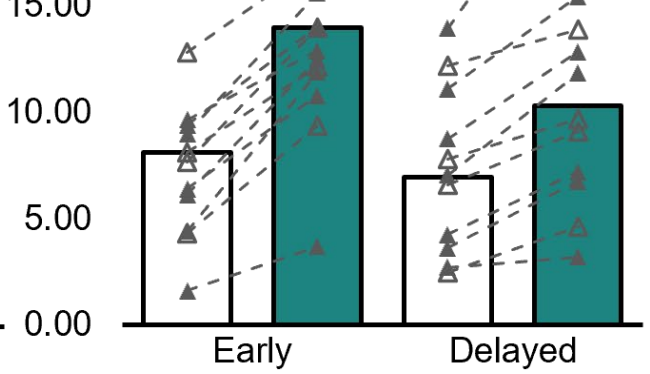

$\square$ Replayed

Fig. 2. Cued reactivation improved episodic recollection. (A) Mean number of Internal details for Experiment 1 . Early corresponds to behavioral performance measured during and immediately after HippoCamera use. Delayed corresponds to behavioral performance after a 3-month delay, during which time participants did not have access to their memory cues. (B) Mean number of Internal details for Experiment 2. Early corresponds to behavioral performance measured shortly after HippoCamera use. Delayed corresponds to behavioral performance after a 3-month delay, during which time participants did not have access to their memory cues. Open triangles reflect Experiment 2 participants who failed the Montreal Cognitive Assessment. ${ }^{* *}=\mathrm{P}<.001$. 
Experiment 2. Experiment 2 was designed with two goals in mind. First, we wanted to replicate findings revealed in Experiment 1 using a condition that reflected longterm autonomous HippoCamera use. To this end, an independent group of participants ( $n=12$, mean age $66.7 \pm 0.81$ SEM, 6 women) used HippoCamera for 10 consecutive weeks (Fig. 1D). They were encouraged to record one event per day and view one replay session per day. Compared to Experiment 1, this protocol better approximated how older adults might use a digital memory aid outside of an experimental context. Compliance was high with an average of $0.95 \pm 0.07$ SEM cues recorded per day and $1.05 \pm 0.03$ SEM replay sessions viewed per day. Cues in the Replayed condition were viewed an average of $7.8 \pm 0.53$ SEM times over the 70 -day use period. By design, the mean number of replays per cue was comparable across experiments (Experiment 1 mean $=8.7$ ) but distributed over a longer period in Experiment 2.

Our second goal was to establish a purer Baseline condition against which Replayed memories could be compared. Anecdotal evidence from Experiment 1 suggested that participants occasionally expected and/or hoped to replay cues that were in the Baseline condition, which reflects some degree of unintended memory retrieval/reactivation. Moreover, randomly interleaving assignment of cues to the Replayed and Baseline conditions in Experiment 1 may have led to an undesirable bleed over effect whereby Replayed cues trigger retrieval of Baseline memories if the events in question happened near together in time. These factors may have inflated the number of event-specific details that were recalled for Baseline events in Experiment 1. As such, HippoCamera cues in Experiment 2 were assigned to either the Replayed or Baseline condition in a predictable, blocked manner, rather than the randomly interleaved assignment used in Experiment 1. Blocked condition assignment alternated across weeks and was counterbalanced across participants. All participants were explicitly informed of these weekly condition switches to ensure that they never anticipated replay of cues in the Baseline condition.

Early memory testing was completed seven days after the 10-week HippoCamera use period. Delayed testing was completed approximately 3.25 months after Early testing. We tested memory for the same subset of events at Early and Delayed testing (the mean 
number trials was identical across Replayed and Baseline conditions: $M=19.7 \pm 0.33$ SEM). Our behavioral results replicate and extend those from Experiment 1. The benefit of repeated replay on episodic recollection of real-world events was apparent in both the Early and Delayed testing periods (Fig. 2B). A Poisson generalized linear mixed model revealed that more Internal details were recalled in the Replayed condition than in the Baseline condition (main effect of condition: $b=0.250, S E=0.0259, z=9.660, P<.001$ ), as well as at the Early memory test relative to the Delayed memory test (main effect of test time point: $b=-0.1240, S E=0.0324, z=-3.840, P<.001$; Table S1). There was also a significant interaction between condition and test time point $(b=-0.0369$, SE $=0.0134$, $z=-2.752, P=.006)$, driven by the fact that the difference in Internal details between Replayed and Baseline events was larger at Early testing $(b=0.574, \mathrm{SE}=0.0601, z=$ $9.549, \mathrm{P}<.001)$ than it was at Delayed testing $(b=0.427, \mathrm{SE}=0.0565, z=7.560, p<$ .001). Proportionally, these differences can be quantified as an $83.8 \%$ increase in the number of event-specific details recalled for the Replayed as compared to the Baseline condition at Early testing, and a $56.0 \%$ increase for the same comparison at Delayed testing. As was the case in Experiment 1, the benefit of replay on recall of Internal details was observed in every participant. This pattern of results was also obtained after adjusting for verbal output (SI Appendix 2.5, Table S5) $(48,49)$. Moreover, replay benefitted all subtypes of Internal details (Internal Event, Time, Place, Perceptual, and Thought/Emotion; SI Appendix 2.3, Fig. S2; Table S2-3). Notably, significantly more event-specific details were recalled in Experiment 2 relative to Experiment 1 (main effect of experiment: $b=0.376$, SE $=0.0792, z=4.751, p<.001$ ), suggesting that cognitive behavioral interventions aimed at improving autobiographical memory may benefit from targeting a limited number of higher-quality events per day and distributing review over a longer period.

Four of the 12 participants from Experiment 2 failed the MoCA, suggesting that they may be showing early signs of cognitive decline. It is worth noting that replay enhanced episodic recollection of event-specific details in each of these individuals at Early and Delayed testing (open triangles in Fig. 2B). On average, $71 \%$ and $41 \%$ more Internal details were recalled for Replayed as compared to Baseline at Early testing and Delayed testing, respectively. At another level, one participant who passed the MoCA 
produced considerably more details than their peers. We note, however, that this individual did not show the largest proportional difference between Baseline and Replayed; the group average was $83.8 \%$, the range was $33.7 \%-170.45 \%$, and the subject in question had an increase of $90.4 \%$, which was $5^{\text {th }}$ highest of the 12 participants. As such, excluding this participant from our statistical analysis did not eliminate or even weaken the effect.

We did not find any evidence for differences in recall of External details across conditions (SI Appendix 2.1, SI Appendix 2.4, Fig. S1, Fig. S3, Table S1, Table S3-4), suggesting that replaying autobiographical memory cues does not influence subsequent retrieval of semantic information. This result is consistent with findings from Experiment 1 , and the broader notion that semantic knowledge tends to be relatively stable (8). We did find that the number of External details recalled differed significantly across Early and Delayed testing (main effect of test time point: $b=-0.146$, SE $=0.0355, z=-4.104, p<$ .001), reflecting the fact that participants recalled significantly more External details at Early testing than they did at Delayed testing. Although we did not predict this outcome, we speculatively suggest that there may be some dependency between Internal and External details, such that a high number of Internal details is accompanied by a relatively high number of External details that situate the target event in a broader context. Together, these findings converge with those from Experiment 1 and suggest that HippoCamera specifically enhances recall of episodic autobiographical information.

As was the case in Experiment 1, we also revealed a main effect of condition on In-Cue detail counts $(b=-0.194, \mathrm{SE}=0.0624, z=-3.111, \mathrm{P}=.00186$; SI Appendix 2.2, Fig. S1), such that participants described fewer In-Cue details for Replayed memories than they did for Baseline memories. These results indicate, again, that repeatedly replaying HippoCamera cues makes participants less reliant on these cues at retrieval and more reliant on elaborative episodic recollection.

\section{Cued Reactivation Evoked More Positive Autobiographical Sentiment at Retrieval}

Having revealed that using HippoCamera to replay autobiographical memory cues selectively increased recall of event-specific episodic details, we next asked whether 
there were qualitative differences in the kind of language used to describe Replayed and Baseline memories (Fig. 3). To examine whether cued reactivation was associated with more positive memory-based event descriptions, we used a text-based sentiment analysis (Valence Aware Dictionary and sEntiment Reasoner; VADER) (50). This approach uses natural language processing to identify subjective states and quantify their polarity (i.e., positivity and negativity). As an example, the statement "We had an amazing time, and Felix was overjoyed when he hit the ball" will receive a more positive score than "We had a nice time, and Felix was pleased when he hit the ball". Similarly, "We had a terrible time, and Felix was devastated when he struck out" will be scored more negatively than "We had a bad time, and Felix was disappointed when he struck out".

We used paired-samples t-tests to probe for differences between normalized composite sentiment scores that capture overall positivity and negativity for Replayed and Baseline memories. For Experiment 1, this approach revealed that sentiment scores were significantly more positive for Replayed memories than Baseline memories at Early testing $(t(21)=2.54, \mathrm{P}<.01, d=0.54)$ and Delayed testing $(t(19)=2.11, \mathrm{P}<.05, d=$ $0.47)$. A similar result was obtained for Early testing in Experiment $2(t(11)=2.42, \mathrm{P}<$ $.05, d=0.70)$. This effect did not persist, however, at Delayed testing in Experiment 2 $(t(11)=0.28, \mathrm{P}=0.4, d=0.08)$. Refer to SI Appendix 2.6 and Fig. S4 for sentiment frequency distributions (Fig. S4). Comparing across experiments, we found that Experiment 2 was generally associated with higher sentiment scores than Experiment 1 $(t(32)=3.68, \mathrm{P}<.001, d=1.32)$. This difference may reflect an increase in the importance of the events recorded in Experiment 2, for which we encouraged participants to record just one event per day. 
A

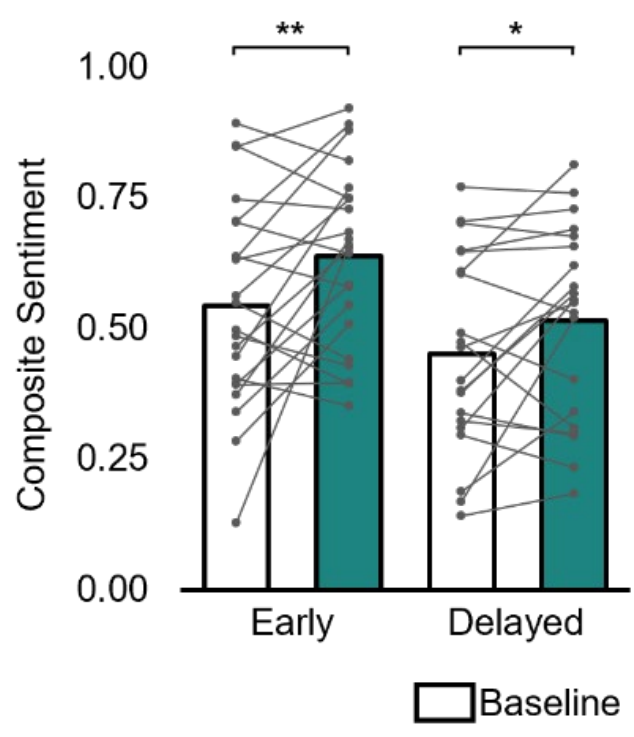

B Experiment 2

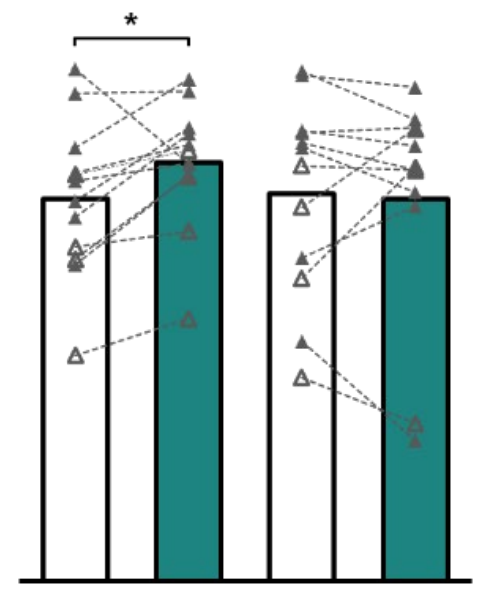

Early

Delayed

Replayed

Fig. 3. Replay evoked more positive sentiment in autobiographical retrieval. (A) Composite sentiment scores from Experiment 1 and (B) Experiment 2. Composite values range from -1 (negative sentiment) to 1 (positive sentiment). Open triangles reflect Experiment 2 participants who failed the Montreal Cognitive Assessment. ${ }^{*}=\mathrm{P}<.05,{ }^{*}=\mathrm{P}<.01$.

\section{Cued Reactivation Promoted Differentiation of Activity Patterns in the Hippocampus}

Having revealed that replaying autobiographical memory cues enhanced episodic recollection in older adults, we next sought to determine whether this effect was associated with increased differentiation of activity patterns in the hippocampus. To this end, we combined fMRI data $(N=25)$ obtained from participants in Experiment 1 ( $N=13$ of 22 total participants) and Experiment $2(\mathrm{~N}=12$ of 12$)$. For both experiments, cues were replayed a comparable number of times (Experiment $1=8.64$; Experiment $2=7.77$ ) and fMRI scanning was completed seven days after the Early autobiographical memory test (Fig. 2A, 2C). In Experiment 1, our recruitment efforts focused on finding older adults who were willing to use the HippoCamera application and visit our laboratory on a regular basis; willingness to be scanned was not a requirement for participation and several of our participants had medical implants (e.g., pacemaker), claustrophobia, arthritis, or other exclusions that made scanning not possible. For Experiment 2, we completed extensive 
pre-screening for all participants, including mock scanning sessions, to ensure that they would be able to complete the fMRI component of our experiment.

Each fMRI scanning session was designed to measure brain activity related to memory for the participant-specific events that had previously been probed in the Early autobiographical memory test. We did this using three task components: Watch Cue, Mentally Relive, and Episodic Probe (Fig. 4A). The Mentally Relive task component was unique to Experiment 2. During the Watch Cue stage participants watched and listened to one of their cues without having to make a behavioral response. After briefly fixating a centrally presented cross, they were then asked to Mentally Relive the event that was just cued in an unconstrained manner (51). They were instructed to do this by recollecting details from the cued event rather than simply visualizing the cue that was just played. No behavioral response was required during this stage. After another brief fixation, they completed an Episodic Probe task that involved making a "yes"/"no" judgment in response to a centrally presented probe word that referred to a person, place, thing, or action that the participant previously recalled during the Early memory test. A given probe could be either a target or a lure. Targets reflected details that were true of the event in question. For example, we would use "KITE" as a target probe for a cue in which a boy is playing baseball if a participant previously recalled that her grandson was distracted by a kite flying in the park near the location of the game. Lures reflected details that were plausible but to our knowledge not true of the event in question. For example, we would use "LILLIAN" as a plausible lure for the baseball cue because the participant mentioned meeting this person when recalling a different event. This procedure (i.e., Watch Cue, Mentally Relive, and Episodic Probe) was repeated once for each tested memory, with repetitions appearing in separate runs. For the episodic probe task, one instance used a target probe word and the other used a lure. Participants correctly responded 'yes' to $86 \%$ \pm 0.08 SEM of the target trials, and 'no' to $79 \% \pm 0.11$ SEM of the lure trials, indicating that our probes were successful in promoting recollection of event-specific details $(82.5 \%$ overall accuracy). 


\section{A fMRI Task}

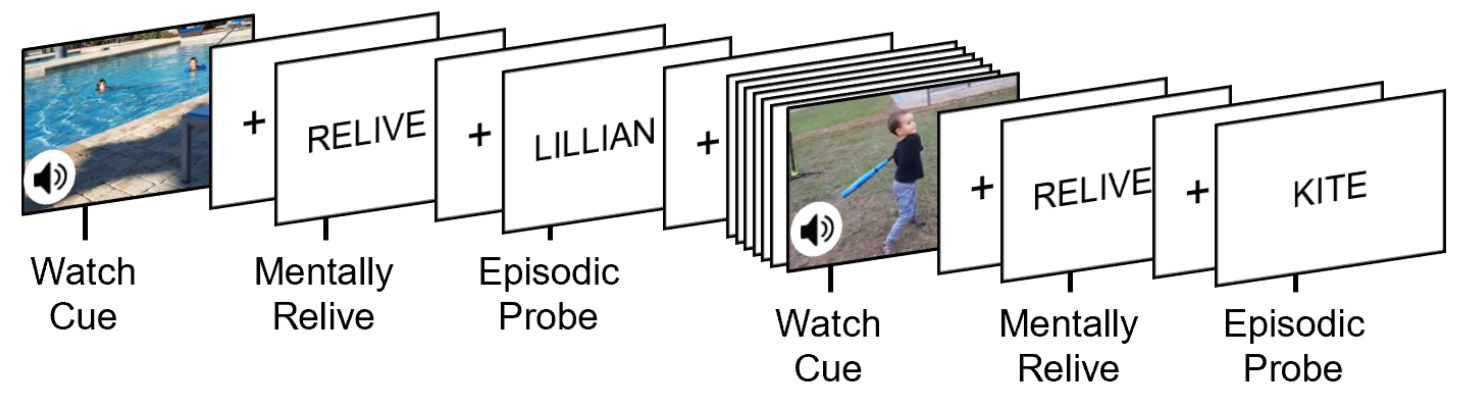

\section{B Differentiation of Hippocampal Activity}

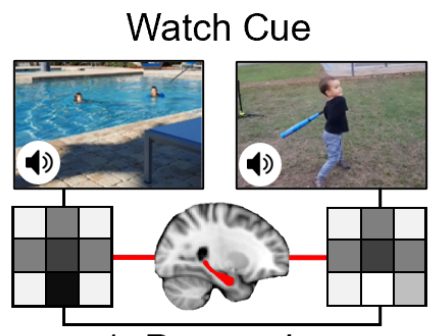

1- Pearson's $r$

C

1.10

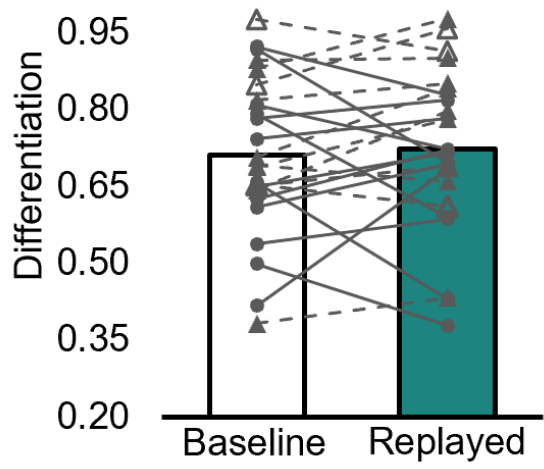

Mentally Relive

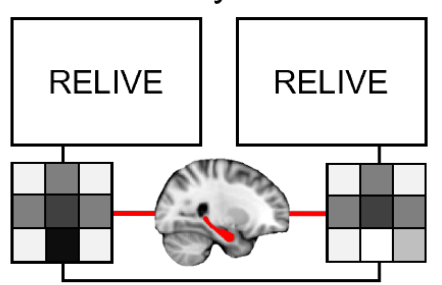

1- Pearson's $r$

Mentally Relive

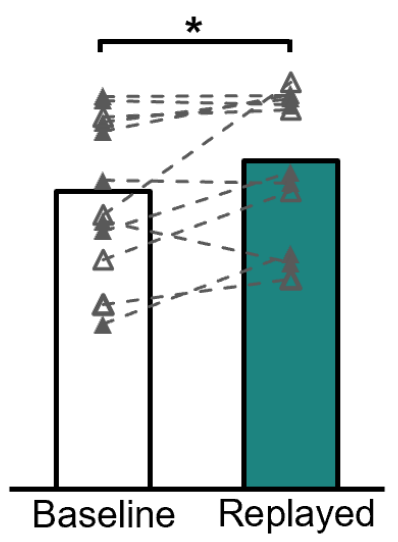

Episodic Probe

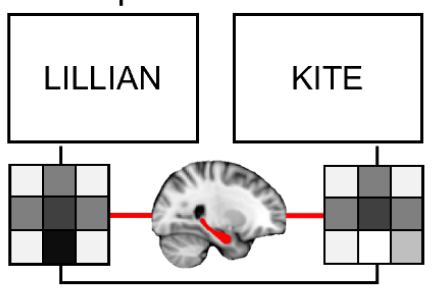

1- Pearson's $r$

\section{Episodic Probe}

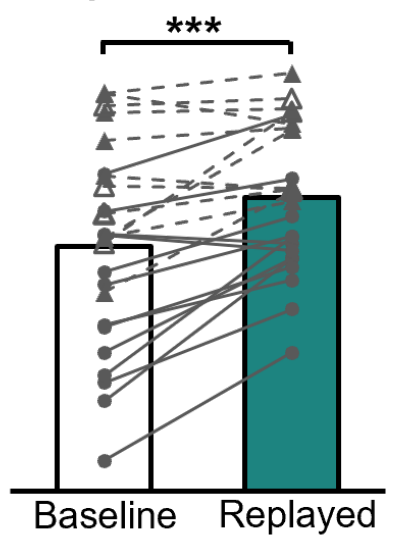

Fig. 4. Replay enhanced differentiation of activity in the hippocampus. (A) Each fMRI trial consisted of three phases. (1) Watch Cue (8s): view a HippoCamera audio-video cue, (2) Mentally Relive (8s; Exp. 2): engage in elaborative recall of the event, and (3) Episodic Probe (4s): judge whether a probe word captured an aspect of the event. (B) For each phase, we quantified differentiation (1 - Pearson's r) between mean activity patterns obtained for all pairs of Replayed memories and between all pairs of Baseline memories. (C) Differentiation scores for each task phase of the fMRI task. Circle markers and solid lines depict data from Experiment 1, whereas triangle markers and dashed lines depict data from Experiment 2. Open triangles reflect Experiment 2 participants who failed the Montreal Cognitive Assessment. ${ }^{*}=\mathrm{P}<.05,{ }^{* * *}=\mathrm{P}<.001$. 
We quantified differentiation of hippocampal activity patterns within each experimental condition separately using a representational similarity analysis (52) (Fig. $4 B$ ). Briefly, single-trial activity was estimated using a general linear model and extracted as spatially distributed patterns across the hippocampus. Activity patterns were averaged across two trials for each event cue, resulting in separate memory-specific estimates for the Watch Cue task component, the Mentally Relive task component, and the Episodic Probe task component. For each of these tasks we first quantified similarities between all pairs of Replayed memories and between all pairs of Baseline memories using Pearson's $r$. These values were then subtracted from one and averaged to produce global measures of differentiation for Replayed and Baseline activity separately (Fig. 4C) (40).

Our results indicated that using HippoCamera to replay autobiographical memory cues fundamentally altered the representational structure of episodic information in the hippocampus by promoting differentiation of memory-related activity patterns. A linear mixed model revealed a significant main effect of condition (Replayed vs. Baseline: $b=$ $\left.2.865 \times 10^{-2}, \mathrm{SE}=6.846 \times 10^{-3}, t(24)=4.184, \mathrm{P}<.001\right)$ and a significant interaction between condition and task $(F(2,2186)=13.932, \mathrm{P}<.001$; SI Appendix 3.5, Table S6-7). This was primarily driven by increased differentiation in hippocampal activity patterns for Replayed compared to Baseline events during the Episodic Probe task $(b=0.0940, S E=0.0157$, $t(45)=5.981, P<.001)$ and the Mentally Relive task $(b=0.0641, \mathrm{SE}=0.0204, t(101)=$ 3.146, $P=.0022)$. We found no evidence for a difference between Replayed and Baseline differentiation during the Watch Cue task $(b=0.0138$, SE $=0.0157, t(45)=0.875, \mathrm{P}=$ .386). Nor did we find evidence for a significant main effect of task (Watch Cue vs. Mentally Relive vs. Episodic Probe: $F(2,11)=1.532, P=.257)$. The condition by experiment interaction term was not significant $(F(1,23)=2.42, P=0.13)$, suggesting that methodological differences across experiments, such as the inclusion of the Mentally Relive task in Experiment 2, did not influence the degree of differentiation across conditions. Moreover, restricting our analyses to activity from correct Episodic Probe trials did not change the overall pattern of results for this task component (Mean Baseline differentiation was .68 for all trials and .67 for correct trials; Mean Replayed differentiation was .77 for all trials and .78 for correct trials). 
To provide a more comprehensive picture of the effect that replay had on activity patterns in the hippocampus, we probed for potential differences in differentiation across the hippocampal long axis (53, 54; SI Appendix 4.1, Fig. S6, Table S6-7). Briefly, this analysis revealed greater differentiation for Replayed as compared to Baseline trials in the anterior and posterior hippocampus for the Mentally Relive and Episodic Probe tasks. However, there were no significant interactions between ROI (anterior vs. posterior hippocampus) and either condition or task. In a second set of exploratory analyses, we asked whether the effect of replay on neural differentiation was unique to the hippocampus. To this end, we quantified differentiation in six structures that have been implicated in episodic memory processing $(55,56)$ : ventromedial prefrontal cortex, anterior cingulate, posterior cingulate, angular gyrus, precuneus, and parahippocampal cortex. We also examined differentiation in a control region that has not been reliably linked to autobiographical memory (postcentral gyrus, i.e., primary somatosensory cortex). Briefly, we found marginal evidence for a main effect of condition (Replayed > Baseline) on differentiation scores in vmPFC $(P=0.02)$, Posterior Cingulate $(P=0.02)$, and Precuneus $(P=.05)$. However, none of these effects survived correction for multiple comparisons (SI Appendix 4.2, Fig. S6, Table S6-7). Thus, replaying cues with HippoCamera produced robust increases in neural differentiation in the hippocampus and marginal evidence for increased differentiation in a subset of cortical areas that have been linked to autobiographical memory retrieval.

\section{Hippocampal Differentiation Was Positively Correlated with Episodic Recollection}

The degree of hippocampal differentiation of an event representation was positively correlated with the recollection of that event's details on the unscanned Early and Delayed autobiographical memory tests (Fig. 5). Memory-specific differentiation scores were estimated by calculating mean pairwise pattern dissimilarities between a given memory and all other memories within the same condition. We then computed the correlation (Pearson's $r$ ) between hippocampal differentiation and recall of Internal details across trials for each participant separately. Because there is no principled reason to believe that the relationship between hippocampal differentiation and recall behavior differ qualitatively across our experimental conditions, we did not distinguish between Replayed 
and Baseline trials for the purpose of this analysis. Directional one-sample $t$-tests against chance (i.e., correlation equal to zero) were performed using within-subject Fisher ztransformed correlation values. Using this approach, we found that degree of hippocampal differentiation measured during the Episodic Probe component of the fMRI task was positively correlated with number of Internal details recalled at Early testing $(t(24)=3.97, \mathrm{P}<.001, d=0.795)$ and Delayed testing $(t(22)=2.51, \mathrm{P}<.01, d=0.523)$. A similar result was obtained for the Mentally Relive component used in Experiment 2 at Early testing $(t(11)=3.45, \mathrm{P}<.01, d=0.997)$ and Delayed testing $(t(11)=3.04 \mathrm{P}<.05$, $d=0.877)$. We also found above chance correlations between differentiation at the Watch Cue component and the number of Internal details recalled at Delayed testing $(t(22)=$ 2.76, $\mathrm{P}<.05, d=0.576$ ). Conversely, we found limited evidence for any meaningful associations between degree of hippocampal differentiation and recall of External details (SI Appendix 4.3, Fig. S7). Taken together, results from our pattern dissimilarity analyses revealed that replaying rich autobiographical memory cues promoted hippocampal differentiation, which in turn was positively correlated with recollection of event-specific episodic details on unscanned autobiographical memory tests administered one week earlier and three months later. 


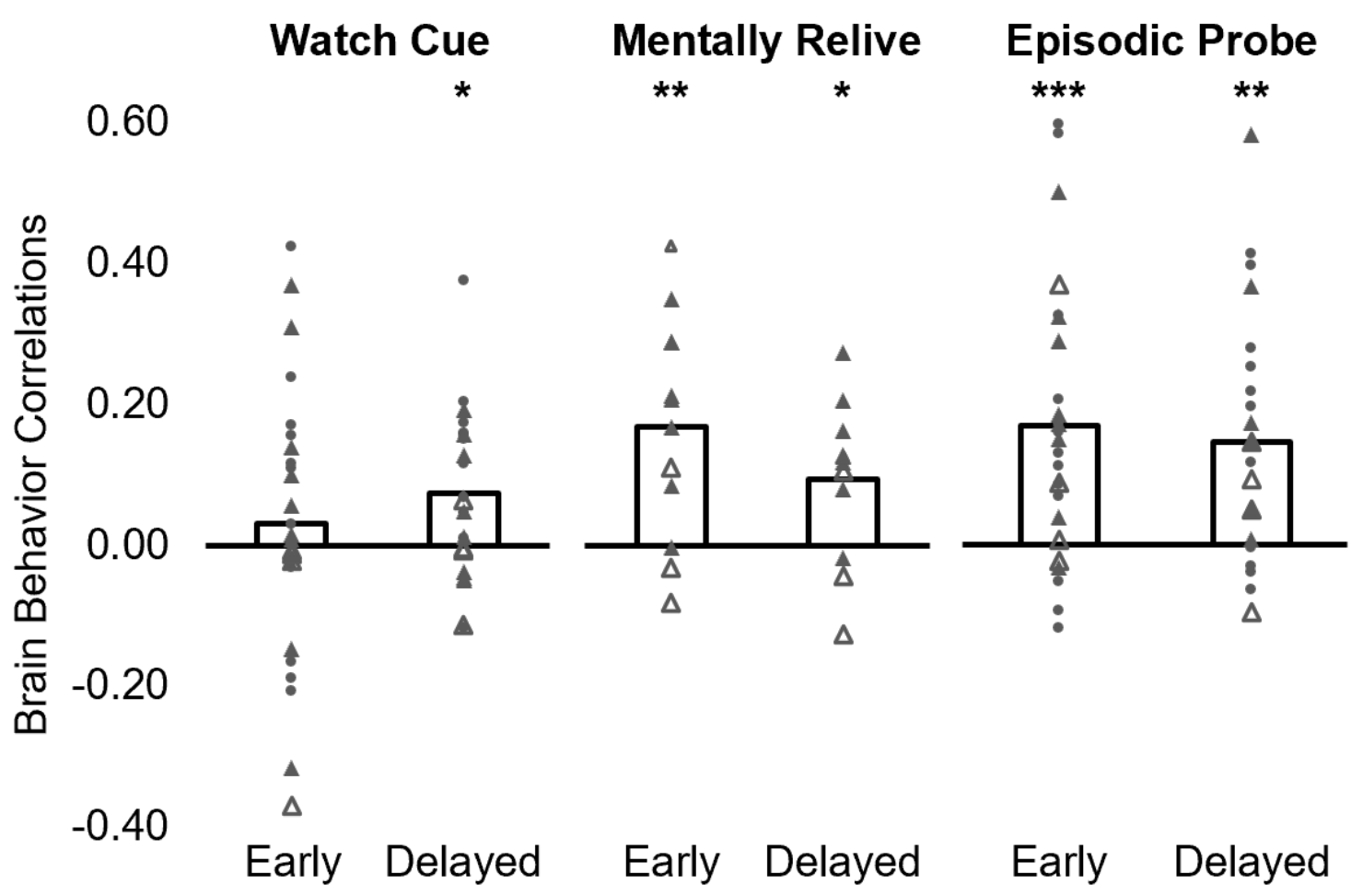

Fig. 5. Degree of hippocampal differentiation is positively correlated with recollection of eventspecific Internal details. Correlations (Pearson's r) between hippocampal differentiation and recall of Internal details during the Early and Delayed autobiographical memory tests. Each marker reflects the Fisher-z transformed $r$ value obtained for one participant. Note that the Mentally Relive component was unique to Experiment 2. Circles reflect participants from Experiment 1. Triangles reflect participants from Experiment 2. Open triangles denote Experiment 2 participants who failed the Montreal Cognitive Assessment. Significance values indicate correlations greater than chance, i.e., correlation of zero, at the group level. ${ }^{*}=\mathrm{P}<.05,{ }^{* *}=\mathrm{P}<.01,{ }^{* * *}=\mathrm{P}<.001$.

\section{Discussion}

We have developed a smartphone-based intervention that uses self-generated, high-fidelity cues to improve memory for everyday events in older adults. Across two experiments, we found that repeatedly replaying rich autobiographical memory cues improved detail-rich recollection shortly after a 14-day (Experiment 1) and a 70-day (Experiment 2) period during which participants used the application to record and repeatedly replay personally meaningful moments from their daily lives. This behavioral enhancement was also evident when memory was assessed a second time, three months after participants stopped using the application. Moreover, replaying memory cues 
evoked more positive autobiographical sentiment at the time of retrieval. We used a pattern-based analysis approach with fMRI data to reveal changes in hippocampal activity related to our cued-reactivation protocol. This approach revealed increased differentiation of activity in the hippocampus related to memories for events that were previously replayed as compared to those that were recorded but never replayed. The extent of differentiation of memory-specific activity in the hippocampus was positively correlated with behavioral measures of episodic re-experiencing. Together, these findings support the conclusion that recording and replaying autobiographical memory cues can enhance episodic recollection by promoting differentiation of underlying representations in the hippocampus.

Replaying autobiographical memory cues enhanced recollection of event-specific details in older adults. Specifically, using HippoCamera to record and replay real-world memory cues generated a 56\% increase in detailed episodic recollection after 14 days of use (Experiment 1), and an 84\% increase after 70 consecutive days of use (Experiment 2). Importantly, the behavioral advantage of replay was apparent in every participant. This effect was selective in that our replay protocol did not consistently affect retrieval of semantic information (Fig. S1, Fig. S3). The significance of these results is apparent against a background of prior research that has revealed age-related declines in the episodic component of autobiographical memory $(8,57,58)$. Our results indicate that replaying brief but detail-rich cues from daily life can combat the tendency for recall in older adults to be limited to high-level episodic details by helping to preserve the eventspecific information that enables mnemonic discrimination among multiple related episodes. The examples "Felix played baseball" and "Felix played baseball and I was thrilled to see the joy on his face the first time he hit the ball" captures this difference between recall of episodic gist versus recall that is characterized by detail-rich mnemonic elaboration. Moreover, this replay-related enhancement persisted after a three-month delay between using HippoCamera and our second autobiographical memory assessment. Accordingly, by contributing to the preservation and accessibility of detailrich memories, our cognitive behavioral intervention helps to bridge the present with the episodic past in older individuals. In particular, vivid, detail-rich retrieval establishes a more stable and reliable connection between the present self and past self than does 
retrieval that is deprived of event-specific details. To the extent these connections contribute to the maintenance of personal identity, we believe that the memory enhancements revealed here are meaningful $(1,2)$.

How does replaying real-world autobiographical memory cues improve episodic recollection? Remembering an event influences the probability and quality of future recollection (19-21), and successful retrieval is thought to beget future retrieval success, while incomplete or failed retrievals reduce the likelihood of future retrieval success (2228). Our behavioral results are consistent with the idea that replaying high-fidelity, selfgenerated cues from everyday events evokes strong reactivation of event memories and therefore strengthens associations among episodic details. Moreover, building on prior laboratory-based work that examined episodic memory for pairs of object or video clips, they reveal a link between memory reactivation and enhanced recollection of complex and dynamic autobiographical events. We obtained this result despite using a cuedreactivation protocol that was not accompanied by explicit retrieval demands, which have been shown to improve subsequent memory relative to mere re-exposure of previously encountered information $(21,59)$. Although this study was not designed to reveal potential differences between the effect of re-exposure and retrieval demands on subsequent memory, we note that we did observe improved episodic recollection of event details even after excluding In-Cue details (i.e., details that were captured by either the audio or video component of the memory cues; Fig. 2, Fig. S1). This observation suggests that reexposure to sufficiently rich cues can improve memory for un-cued information about the cued event even in the absence of any retrieval demands.

Results from our pattern similarity analyses indicated that replaying detail-rich cues increased differentiation of memory-related activity in the hippocampus. In other words, repeated reactivation minimized similarities between episodic memory representations by reducing representational overlap in the hippocampus. Importantly, the degree to which a given memory representation was differentiated predicted the quality of episodic recollection, such that greater dissimilarity was associated with retrieval of more eventspecific details. By linking the differentiation of activity in the hippocampus to enhanced episodic recollection, our results dovetail with evidence from computational modeling 
suggesting that greater differentiation among memory representations can reduce competition at retrieval and therefore facilitate detail-rich recollection $(23,60)$. The fact that increased differentiation was driven by HippoCamera replay after initial encoding suggests that our results cannot be fully explained by the notion that the hippocampus minimizes overlap among memory representations at the time of encoding (i.e., patternseparation; 61-63). Accordingly, we believe that using HippoCamera to replay memory cues differentiates underlying representations in a manner that protects against the postencoding loss of detail-based information.

The impact of episodic memory loss is far-ranging and includes reductions in one's sense of identity, confidence, and autonomy (1, 2, 43). Because HippoCamera was designed to enhance episodic memory in older adults, we also asked whether replaying would evoke more positive autobiographical sentiment at the time of retrieval. Although we did not assess mood or screen for depressive disorders in this study, we reasoned that replay would encourage repeated positive reminiscence and that this would be reflected in improved autobiographical sentiment in our behavioral memory assessments (e.g., Baseline retrieval: "We were at the park for almost the entire day" vs. Replayed retrieval: "We were at the park for almost the entire day and it was just lovely"). Our results indicated that this was indeed the case, as sentiment scores were significantly more positive for Replayed as compared to Baseline memories at Early and Delayed testing in Experiment 1 and at Early testing in Experiment 2. When considered in the context of a broader literature that links memory impairment to mood disorders, including depression $(42,44)$, these results suggest that using HippoCamera as a memory aid may contribute to emotional well-being.

Although the current set of experiments was designed to characterize the effect of HippoCamera replay on memory, it is worth noting that this memory aid also enriches encoding in several important ways. As such, because the Replayed and Baseline conditions had identical encoding protocols, events in both conditions were processed at encoding in a fundamentally different way from the incidental manner by which we typically learn about the world and encode new events. By incentivizing the creation of memory cues, HippoCamera encourages a shift from incidental encoding to intentional 
encoding, which is known to improve retrieval success in older adults (64). Anecdotally, many of our participants reported increased awareness of the world around them (e.g., one individual described their experience as a participant as follows, "... It was very motivational. I started to have more confidence in myself and started to be more aware of things around me. I think it made my memory go up a shot."). Creating self-generated memory cues also promotes deep processing of event details and meaning, which HippoCamera achieves by requiring a brief verbal description of the event, a video that captures diagnostic perceptual information, and a rating of the personal significance of the event (Fig. 1A). Importantly, this deep processing ensures correspondence between the retrieval cue and the information encoded in memory (15). Lastly, numerous participants reported having varied their behavioral repertoire out of a desire to record and replay interesting content. For example, one participant noted "... Sometimes I would be sitting at home and realize that I needed to film something so I would go out to the library or the church just to have something to do". Considering these points together, there was likely enriched encoding of events in both Replayed and Baseline conditions, and so we likely underestimated the beneficial effects of using HippoCamera when we contrasted Replayed and Baseline conditions. Ultimately, further research is required to isolate and quantify the contribution of enhanced encoding, perhaps through comparison of HippoCamera with a passive photograph system (i.e., wearable camera). At another level, we also note that it is not clear how best to define a baseline condition when conducting research outside of a controlled laboratory environment. We opted for a relatively conservative approach to ensure that we did not overestimate the effect of repeated replay.

This set of experiments, and HippoCamera more generally, builds on over a decade of research that has examined the efficacy of wearable cameras as memory aids in both healthy and cognitively impaired populations (46, 65-74; for review, see 47, 75, 76). Wearable cameras, which passively capture photographs for later review, provide richer, more voluminous information about past events than more traditional memory aids such as written diaries. Indeed, reviewing such photographs improves episodic memory for everyday experiences relative to review of diary entries, and modulates activity in key memory-related brain areas, including the hippocampus $(47,75)$. Despite these promising 
outcomes, wearable cameras present practical barriers that make it difficult for older adults and individuals with memory impairment to easily integrate them into their daily life. First, they require companion hardware (e.g., a computer) and the technical proficiency to transfer still photographs for review. Second, the review process is time consuming as it can involve hundreds to thousands of indiscriminately captured photographs, creating an inefficient user experience and caregiver burden that compounds as cues accumulate over time. HippoCamera removes these practical barriers by enabling high-quality cues to be recorded and replayed on the same device, and by coupling automatically curated replayed sessions with periodic notifications reminding users to record and review their memory cues. Moreover, neither wearable camera technology nor popular social media platforms focus on enriching the encoding experience. HippoCamera is, to our knowledge, the only digital memory application that overtly promotes deep encoding strategies. At another level, few studies conducted with wearable cameras have deeply probed memory quality to determine whether the beneficial effects of photograph review extend beyond improved recognition of previously studied images. Here, we adapted Autobiographical Interview scoring by discounting In-Cue details to demonstrate that repeatedly replaying HippoCamera memory cues improved memory for unstudied aspects of everyday experiences. Moreover, extant fMRI research that has used pictures from wearable cameras as stimuli has not asked the theoretically important question of how picture review improves memory (e.g., via pattern differentiation, integration of neural representations, or gain modulation). Using HippoCamera, we have revealed novel evidence that points to replay-related memory differentiation as a neural mechanism that can explain improvements in recall of episodic event details.

Is replaying with HippoCamera superior to reviewing non-HippoCamera content captured using a smartphone or camera? We think so. First, the guided recording process means that memory encoding and retrieval is intentional: participants are encouraged to pay greater attention to the events of their lives and generate cues that are specifically designed to trigger later retrieval. Second, our application curates replay sessions with powerful memory cues by guiding users to systematically reflect on their past in a highly structured and targeted way. Third, having a dedicated application with daily targets and notifications that remind participants to record and replay cues reinforces memory- 
oriented behavior and cognition in a manner that casual smartphone use does not. Compliance often presents a considerable barrier in the context of cognitive behavioral interventions in older adults (77). It is noteworthy that we obtained high compliance in every one of our older adult participants in terms of meeting daily record and replay expectations in Experiment 1 and Experiment 2.

A key finding revealed here is that repeated replay of HippoCamera cues strengthens memories in a manner that enhances detail-rich retrieval and increases differentiation among activity patterns in the hippocampus. A notable limitation of this study that impacts on interpretation of these results is the fact that these replay-related benefits were obtained using well-learned cues to prompt retrieval (i.e., previously replayed HippoCamera cues). It is thought that retrieval reflects an interaction between a cue and an established memory trace (i.e., ecphory; 78, 79). This raises the important question of whether the memory strengthening we observed reflects a narrow benefit that is obtained only when prompted by a previously viewed HippoCamera cue, or if this effect generalizes to other non-practiced cues and/or retrieval contexts. At a minimum, we can make the strong claim that replaying HippoCamera memory cues increases ecphory when those precise cues are later used to trigger retrieval. However, we have not systematically asked whether the benefit of replay is attenuated when using test cues that are related to but different from those that were replayed with HippoCamera. Nevertheless, data from a single-case study conducted with a hippocampal amnesic patient speaks to this issue (SI Appendix 5, Fig. S8, Fig. S9). Notably, while she could not recall any episodic details for 3-week old events memorialized in her diary, she produced some details for 3-week old events recorded and repeatedly replayed with HippoCamera. In fact, she recalled some details spontaneously in conversation suggesting that replaying rich, self-generated autobiographical memory cues may have benefits that can be detected without cues.

Finally, we note that $65 \%$ of our participants were women, and that women usually outperform men in episodic memory performance (80). Therefore, to confirm the generality of these findings across genders and sexes, we will need to test these factors as moderators in a larger-sample replay-based memory intervention. 
In sum, we have developed a smartphone-based memory aid that uses cued reactivation of real-world events to improve episodic recollection in older adults. This beneficial outcome was linked to a corresponding increase in the differentiation of activity in the hippocampus and more positive autobiographical sentiment at the time of retrieval. By strengthening connections between the present and past self, this application presents a non-invasive approach to mitigating real-world age-related memory decline. More generally, to the extent that autobiographical memory makes important contributions to other aspects of cognition and the maintenance of meaningful interpersonal relationships, this intervention has potential to promote graceful aging.

\section{Materials and Methods}

Participants. Experiment 1. Twenty-two neurologically healthy older adults (mean age $=69.64$ years \pm 0.89 SEM, range $=62-76$ years, 16 women) participated in Experiment 1. Two participants were unable to complete a Delayed autobiographical memory test. All participants obtained a passing score on the Montreal Cognitive Assessment (MoCA) ( mean score $=27.10 \pm 0.26$ SEM, range $=26-30$ ), suggesting that these individuals were cognitively healthy at the time of testing (81). Of this sample, 13 individuals also participated in the fMRI experiment (mean age $=68.84$ years $\pm 1.19 \mathrm{SEM}$, range $=62-76$ years, 10 women, mean MoCA $=27.62 \pm 0.35$ SEM). Experiment 2. Twelve neurologically healthy older adults participated in Experiment 2 (mean age $=66.77$ years \pm 0.81 SEM, range $=61-71,6$ women, mean MoCA score $=26.58 \pm 0.85$ SEM). Four of these individuals failed the MoCA (scores of 20, 24, 25, and 25) but had no documented history of neurological or cognitive disorder. The research protocol for both experiments was reviewed and approved by the Research Ethics Board at The University of Toronto. Written informed consent was obtained from all participants prior to HippoCamera use and again before fMRI data collection.

HippoCamera Smartphone Application. We developed the HippoCamera application using a participatory design approach aimed at optimizing ease of use and enjoyability in older adults. Cues can be created in approximately 40 seconds and replayed in approximately 60 seconds. All participants were able to easily incorporate the application into their daily routines and most indicated that they enjoyed doing so. As evidence, we 
note that nearly all participants met our expectations in terms of how many cues should be recorded per day and how many replay sessions should be viewed per day in both experiments.

This research was conducted using a beta version of the application developed by our research team. The software was subsequently updated by Tactica Interactive (Winnipeg, Manitoba) to improve stability, enhance visualizations, enable cloud-based storage, and allow researchers to remotely access content recorded by participants. This version of the application can be obtained for scientific purposes from Apple's App Store and Google Play. As of the time of writing, this is a research-dedicated application that requires an access code that can be obtained from a corresponding author.

Recording Cues with HippoCamera: Cues are recorded in a four-step process, with automated transitions between steps (Fig. 1A). First, participants intentionally make the decision to record an ongoing event. Second, they capture an 8-second audio recording that is a self-generated verbal description of the target event. Third, they record a 24second high-definition video. Fourth, they rate the significance of the event using a 1-5 scale. Meta-data are also automatically recorded, including time, date, and GPS coordinates, when available. Upon completing a recording, the application generates integrated cues that combine the verbal description with a speeded version of the video. Audio is stripped from the 24-second video and the resulting file is accelerated by a factor of three, resulting in an 8-second speeded video. The speeded video is then coupled with the audio file containing the 8-second verbal description. A notification system can be toggled on or off to encourage participant compliance. For the current study, these notifications reminded participants to record five cues per day in Experiment 1 and one cue per day in Experiment 2.

Replaying Cues with HippoCamera: Replay takes place within sessions that consist of up to five sequentially presented cues that are automatically selected (Fig. 1B). Cues are separated by a lead-in screen that displays the date and approximate age of the event. Within a session, cues are replayed in reverse chronological order, meaning newer cues are played before older cues. The application is designed to replay cues in a manner that achieves balance between distributed learning and the prioritization of recent, highly 
significant events. For the current research, notifications encouraged participants to replay 6 times per day in Experiment 1 and once per day in Experiment 2.

Replay Instructions: At intake, participants were given guidance regarding when they should initiate replay sessions and how they should engage with replayed content. Specific instructions were developed to standardize our replay protocol as much as is possible in the context of unsupervised, real-world research. We instructed participants to initiate replay sessions at relatively quiet moments to minimize distraction and optimize effortful recollection. Although we did not communicate firm expectations, we suggested that they anchor some of their replay sessions to routine events that often present distraction-free opportunities for replay, such as while drinking coffee in the morning. We also instructed participants to engage in deep, contemplative processing of memories during replay by asking them to actively recollect a specific aspect of each cued event. This approach was motivated by the large body of research that has revealed a clear benefit for active retrieval over mere re-exposure to a stimulus (i.e., the test effect; 82). Lastly, participants were encouraged to engage in a moment of reflection using in-app instructions that were presented on screen at the conclusion of each replay session: "Take a moment to reflect on the memories reviewed". This prompt was designed to give participants an opportunity to contemplate their memories at a more leisurely pace than is possible during the preceding replay session. Moreover, the open-ended nature of the guidance allowed them to flexibly focus on their personal past in a self-directed manner.

Autobiographical Memory Test. Behavioral performance was assessed using an adapted version of the Autobiographical Interview (8) (SI Appendix 1.1.1). Whereas the Autobiographical Interview allows participants to choose which memories to recall, we prompted retrieval by presenting participants with their self-generated HippoCamera cues. Trial order was randomized, and experimenters were blind to the condition of each cue. Verbal responses were recorded, transcribed, and then scored using an adapted version of the Autobiographical Interview protocol (45) (SI Appendix 1.1.2). Specifically, each recalled detail was scored as being either Internal, External, or In-Cue. Internal and External detail counts excluded In-Cue details, meaning descriptions of information that was readily apparent in the cue (either the verbal description or video) did not count 
toward recall. In other words, we conservatively discounted details that a naïve observer could also describe based on having seen only the cue, but not having experienced the event. See SI Appendix 1.1.3 for details regarding stimulus selection.

Sentiment Analysis. Cued-recall transcripts were processed using VADER, a lexicon and rule-based sentiment analysis tool that systematically identifies and quantifies affective states communicated in natural language (50). The polarity and intensity scores of the 9000 words comprising the VADER lexicon reflect sentiment ratings from human observers. VADER generates scores that reflect positive sentiment, negative sentiment, neutral sentiment, and a normalized weighted composite score that ranges between -1 (extremely negative) and 1 (extremely positive). We used composite scores as dependent measures, as they provide a single unidimensional sentiment value. Although In-Cue details were excluded from Internal and External detail counts, they were not excluded for the purpose of performing our sentiment analyses because there is no principled reason to predict that In-Cue details will be described with more positive or more negative valence across conditions.

fMRI Task. Data for Experiment 1 were collected across 4 functional runs. Data from one run could not be collected for one participant due to a technical error. Data for Experiment 2 were collected across eight functional runs. Three participants were unable to complete all eight runs due to physical discomfort: one completed four runs, two completed six runs. Each Experiment 1 trial was 16 seconds in duration and consisted of two sub-tasks (Watch Cue and Episode Probe) separated by baseline fixation (Fig. 4A). More specifically, participants first watched a HippoCamera cue (8s), then fixated on a centrally presented cross (4s), and finally responded to an episodic verification probe (4s). Each Experiment 2 trial was 28 seconds in duration and consisted of three sub-tasks (Watch Cue, Mentally Relive, and Episode Probe) separated by baseline fixation (Fig. 4A). Participants first watched a HippoCamera cue (8s), then fixated on a centrally presented cross (4s), then actively recollected the cued event (8s), then fixated on a centrally presented cross (4s), and finally responded to an episodic verification probe (4s). Trials in each experiment were separated by a jittered baseline fixation that spanned 4-8 seconds in duration. A brief audio tone was played $500 \mathrm{~ms}$ prior to the onset of a new trial. 
Moreover, the onset of each sub-task within a trial was signaled by changing the color of the baseline fixation cross from white to red. All timing parameters were inspired by prior research in which participants viewed and later mentally relived audio-video stimuli that were similar in duration to the HippoCamera cues used here (83).

See SI Appendix 3.1-3.4 for details regarding fMRI data acquisition, preprocessing procedures, ROI definition, and analytical procedures.

\section{References}

1. Conway MA, Pleydell-Pearce CW (2000) The construction of autobiographical memories in the self-memory system. Psychol Rev 107(2):261-288.

2. Addis DR, Tippett $L$ (2004) Memory of myself: Autobiographical memory and identity in Alzheimer's disease. Memory 12(1):56-74.

3. Gaesser B, Schacter DL (2014) Episodic simulation and episodic memory can increase intentions to help others. Proc Natl Acad Sci USA 111(12):4415-4420.

4. Harris CB, Rasmussen AS, Berntsen D (2014) The functions of autobiographical memory: An integrative approach. Memory 22(5):559-581.

5. Pillemer D. (2003) Directive functions of autobiographical memory: The guiding power of the specific episode. Memory 11(2):193-202.

6. Schacter DL, Addis DR, Buckner RL (2007) Remembering the past to imagine the future: the prospective brain. Nat Rev Neurosci 8(9):657-661.

7. Bluck S, Alea N, Habermas T, Rubin DC (2005) A tale of three functions: The selfreported uses of autobiographical memory. Social Cogn 23(1):91-117.

8. Levine B, Svoboda E, Hay JF, Winocur G, Moscovitch M (2002) Aging and autobiographical memory: dissociating episodic from semantic retrieval. Psychol Aging 17(4):677-689.

9. Gorbach T et al (2017) Longitudinal association between hippocampus atrophy and episodic-memory decline. Neurobiol Aging 51:167-176.

10. Cabeza $R$ et al (2018) Maintenance, reserve and compensation: the cognitive neuroscience of healthy ageing. Nat Rev Neurosci 19(11):701-710.

11. Scoville WB, Milner B (1957) Loss of recent memory after bilateral hippocampal lesions. J Neurol Neurosurg Psychiatry 20(1):11-21.

12. Tulving E, Markowitsch HJ (1998) Episodic and declarative memory: role of the hippocampus. Hippocampus 8(3):198-204. 
13. Ebbinghaus, H. (2013). Memory: A contribution to experimental psychology. Ann Neurosci, 20(4):155-156.

14. Craik FI, Lockhart RS (1972) Levels of processing: A framework for memory research. J Verbal Learning Verbal Beh 11(6):671-684.

15. Mäntylä T, Nilsson LG (1988) Cue distinctiveness and forgetting: Effectiveness of self-generated retrieval cues in delayed recall. J Exp Psychol Learn Mem Cogn 14(3):502-509.

16. Tan DS, Stefanucci JK, Proffitt DR, Pausch R (2001) The Infocockpit: Providing location and place to aid human memory. In Proceedings of the 2001 Workshop on Perceptive User Interfaces, ACM: 1-4. https://doi.org/10.1145/971478.971526

17. Robin J, Wynn J, Moscovitch M (2016) The spatial scaffold: The effects of spatial context on memory for events. J Exp Psychol Learn Mem Cogn 42(2):308-315.

18. Skaggs WE, McNaughton BL, Wilson MA, Barnes CA (1996) Theta phase precession in hippocampal neuronal populations and the compression of temporal sequences. Hippocampus 6(2):149-172.

19. McGaugh JL (2000) Memory--a century of consolidation. Science 287(5451):248251.

20. Dudai $Y$, Karni A, Born J (2015) The consolidation and transformation of memory. Neuron 88(1):20-32.

21. Roediger HL, Karpicke JD (2006) Test-enhanced learning: Taking memory tests improves long-term retention. Psychol Sci 17:249-255.

22. Norman KA, Newman EL, Detre G (2007) A neural network model of retrievalinduced forgetting. Psychol Rev 114(4):887-953.

23. Ritvo VJ, Turk-Browne NB, Norman KA (2019) Nonmonotonic plasticity: how memory retrieval drives learning. Trends Cogn Sci 23(9):726-742.

24. St. Jacques PL, Schacter DL (2013) Modifying memory: Selectively enhancing and updating personal memories for a museum tour by reactivating them. Psychol Sci 24(4):537-543.

25. Sinclair AH, Barense MD (2018) Surprise and destabilize: prediction error influences episodic memory reconsolidation. Learn Mem 25(8):369-381.

26. Newman EL, Norman KA (2010) Moderate excitation leads to weakening of perceptual representations. Cereb Cortex 20(11):2760-2770.

27. Detre GJ, Natarajan A, Gershman SJ, Norman KA (2013) Moderate levels of activation lead to forgetting in the think/no-think paradigm. Neuropsychologia 51(12):2371-2388. 
28. Wang TH, Placek K, Lewis-Peacock JA (2019) More is less: increased processing of unwanted memories facilitates forgetting. J Neurosci 39(18):3551-3560.

29. Schlichting ML, Mumford JA, Preston AR (2015) Learning-related representational changes reveal dissociable integration and separation signatures in the hippocampus and prefrontal cortex. Nat Comm 6(1):1-10.

30. Schapiro AC, Kustner LV, Turk-Browne NB (2012) Shaping of object representations in the human medial temporal lobe based on temporal regularities. Curr Biol 22(17):1622-1627.

31. Kyle CT, Stokes JD, Lieberman JS, Hassan AS, Ekstrom AD (2015) Successful retrieval of competing spatial environments in humans involves hippocampal pattern separation mechanisms. Elife 4:e10499.

32. Ritchey M, Montchal ME, Yonelinas AP, Ranganath C (2015) Delay-dependent contributions of medial temporal lobe regions to episodic memory retrieval. Elife 4:e05025.

33. Kim M, Jeffery KJ, Maguire EA (2017) Multivoxel pattern analysis reveals 3D place information in the human hippocampus. J Neurosci 37(16):4270-4279.

34. Tompary A, Davachi L (2017) Consolidation promotes the emergence of representational overlap in the hippocampus and medial prefrontal cortex. Neuron 96(1):228-241.

35. Robin J, Buchsbaum BR, Moscovitch M (2018) The primacy of spatial context in the neural representation of events. J Neurosci 38(11):2755-2765.

36. LaRocque KF et al (2013) Global similarity and pattern separation in the human medial temporal lobe predict subsequent memory. J Neurosci 33(13):5466-5474.

37. Hulbert JC, Norman KA (2015) Neural differentiation tracks improved recall of competing memories following interleaved study and retrieval practice. Cereb Cortex 25(10):3994-4008.

38. Chanales AJ, Oza A, Favila SE, Kuhl BA (2017) Overlap among spatial memories triggers repulsion of hippocampal representations. Curr Biol 27(15):2307-2317.

39. Dimsdale-Zucker HR, Ritchey M, Ekstrom AD, Yonelinas AP, Ranganath C (2018) CA1 and CA3 differentially support spontaneous retrieval of episodic contexts within human hippocampal subfields. Nat Comm 9(1):1-8.

40. Ezzyat Y, Inhoff MC, Davachi L (2018) Differentiation of human medial prefrontal cortex activity underlies long-term resistance to forgetting in memory. J Neurosci 38(48):10244-10254.

41. Brunec IK, Robin J, Olsen RK, Moscovitch M, Barense MD (2020) Integration and differentiation of hippocampal memory traces. Neurosci Biobehav Rev 118:196-208. 
42. Söderlund H, Moscovitch M, Kumar N, Daskalakis ZJ, Flint A, Herrmann N, Levine B (2914) Autobiographical episodic memory in major depressive disorder. J Abnorm Psychol 123(1):51-60.

43. Waters TEA (2014) Relations between the functions of autobiographical memory and psychological wellbeing. Memory 22(3):265-75.

44. Christman S, Bermudez C, Hao L, Landman BA, Boyd B, Albert K, Woodward N, Shokouhi S, Vega J, Andrews P, Taylor WD (2020) Accelerated brain aging predicts impaired cognitive performance and greater disability in geriatric but not midlife adult depression. Transl Psychiatry 10(1):1-11.

45. Renoult $L$ et al (2020) Classification of general and personal semantic details in the Autobiographical Interview. Neuropsychologia 144:107501.

46. Loveday C, Conway MA (2011) Using SenseCam with an amnesic patient: accessing inaccessible everyday memories. Memory 19(7):697-704.

47. Silva AR, Pinho MS, Macedo L, Moulin CJA (2018) A critical review of the effects of wearable cameras on memory. Neuropsychol Rehabil 28(1):117-141.

48. Lockrow AW, Setton R, Spreng KAP, Sheldon S, Turner GR, Spreng RN (2021) Taking stock of the past: a psychometric evaluation of the Autobiographical Interview. bioRxiv https://doi.org/10.1101/2021.12.22.473803

49. Setton R, Sheldon S, Turner GR, Spreng RN (2022) Temporal pole volume is associated with episodic autobiographical memory in healthy older adults. Hippocampus 32(5):373-385.

50. Hutto C, Gilbert E (2014) Vader: A parsimonious rule-based model for sentiment analysis of social media text. In Eighth international AAAl conference on weblogs and social media.

51. Tulving E (1985) Memory and consciousness. Canadian Psychology/Psychologie Canadienne, 26(1):1-12.

52. Kriegeskorte N, Mur M, Bandettini PA (2008) Representational similarity analysisconnecting the branches of systems neuroscience. Front Syst Neurosci 2:4.

53. Poppenk J, Evensmoen HR, Moscovitch M, Nadel L (2013) Long-axis specialization of the human hippocampus. Trends Cogn Sci 17(5):230-240.

54. Brunec IK et al (2018) Multiple scales of representation along the hippocampal anteroposterior axis in humans. Curr Biol 28(13):2129-2135.

55. Svoboda E, McKinnon MC, Levine B (2006) The functional neuroanatomy of autobiographical memory: a meta-analysis. Neuropsychologia, 44(12):2189-2208.

56. Schacter DL, Addis DR, Buckner RL (2007) Remembering the past to imagine the future: the prospective brain. Nat Rev Neurosci 8(9):657-661. 
57. Nyberg $L$ et al (2003) Selective adult age differences in an age-invariant multifactor model of declarative memory. Psychol Aging 18(1):149-160.

58. Rönnlund M, Nyberg L, Bäckman L, Nilsson LG (2005) Stability, growth, and decline in adult life span development of declarative memory: cross-sectional and longitudinal data from a population-based study. Psychol Aging 20(1):3-18.

59. Karpicke JD, Roediger HL (2008). The critical importance of retrieval for learning. Science, 319(5865):966-968.

60. Norman KA, Newman E, Detre G, Polyn S (2006) How inhibitory oscillations can train neural networks and punish competitors. Neural Comput 18(7):1577-1610.

61. Marr D (1971) Simple memory: a theory for archicortex. Philos Trans R Soc Lond Ser B Biol Sci 262:23-81.

62. O'Reilly RC, McClelland JL (1994). Hippocampal conjunctive encoding, storage, and recall: avoiding a trade-off. Hippocampus 4(6):661-682.

63. Yassa MA, Stark CE (2011). Pattern separation in the hippocampus. Trends Neurosci, 34(10):515-525.

64. Light LL, Singh A (1987). Implicit and explicit memory in young and older adults. J Exp Psychol Learn Mem Cogn 13(4):531-541.

65. Hodges S, Williams L, Berry E, Izadi S, Srinivasan J, Butler A, Smyth G, Kapur N, Wood K (2006) SenseCam: A retrospective memory aid. In International Conference on Ubiquitous Computing (pp. 177-193). Springer, Berlin, Heidelberg.

66. Hodges S, Berry E, Wood K (2011) SenseCam: A wearable camera that stimulates and rehabilitates autobiographical memory. Memory 19(7):685-696.

67. Lee ML, Dey AK (2008) Lifelogging memory appliance for people with episodic memory impairment. In Proceedings of the 10th international conference on Ubiquitous computing (pp. 44-53).

68. Berry E, Kapur N, Williams L, Hodges S, Watson P, Smyth G, Srinivasan J, Smith R, Wilson B, Wood K (2007). The use of a wearable camera, SenseCam, as a pictorial diary to improve autobiographical memory in a patient with limbic encephalitis: A preliminary report. Neuropsychol Rehabil 17(4-5):582-601.

69. Berry E, Hampshire A, Rowe J, Hodges J, Kapur N, Watson P, Browne G, Smyth G, Wood K, Owen AM (2009) The neural basis of effective memory therapy in a patient with limbic encephalitis. J Neurol Neurosurg Psychiatry 80(11):1202-1205.

70. Browne G, Berry E, Kapur N, Hodges S, Smyth G, Watson P, Wood K (2011) SenseCam improves memory for recent events and quality of life in a patient with memory retrieval difficulties. Memory 19(7):713-722. 
71. Pauly-Takacs K, Moulin CJA, Estlin EJ (2011) SenseCam as a rehabilitation tool in a child with anterograde amnesia. Memory 19(7):705-712.

72. St. Jacques PL, Conway MA, Cabeza R (2011) Gender differences in autobiographical memory for everyday events: retrieval elicited by SenseCam images versus verbal cues. Memory 19(7):723-732.

73. Crete-Nishihata M, Baecker RM, Massimi M, Ptak D, Campigotto R, Kaufman LD, Brickman AM, Turner GR, Steinerman JR, Black SE (2012) Reconstructing the past: personal memory technologies are not just personal and not just of rmemory. HumanComputer Interaction 27(1-2):92-123.

74. Woodberry E, Browne G, Hodges S, Watson P, Narinder K, Woodberry K (2015) The use of a wearable camera improves autobiographical memory in patients with Alzheimer's disease. Memory 23(3):340-349.

75. Chow TE, Rissman J (2017) Neurocognitive mechanisms of real-world autobiographical memory retrieval: insights from studies using wearable camera technology. Ann N Y Acad Sci 1396(1):202-221.

76. Allé M, Manning L, Potheegadoo J, Coutelle R, Danion J-M, Berna, F (2017) Wearable cameras are useful tools to investigate and remediate autobiographical memory impairment: a systematic PRISMA review. Neuropsychol Rev 27(1):81-99.

77. Boot WR, Champion M, Blakely DP, Wright T, Souders DJ, Charness N (2013) Video games as a means to reduce age-related cognitive decline: attitudes, compliance, and effectiveness. Front Psychol 4(31).

78. Semon R. The Mneme (G. Allen \& Unwin, 1921).

79. Josselyn S, Köhler S, Frankland PW (2015) Finding the engram. Nat Rev Neurosci 16(9):521-534.

80. Herlitz A, Rehnman J (2008) Sex differences in episodic memory. Curr Dir Psychol Sci 17(1):52-56.

81. Nasreddine ZS et al. (2005). The Montreal Cognitive Assessment, MoCA: A brief screening tool for mild cognitive impairment. J Am Geriatr Soc 53(4):695-699.

82. Roediger III HL, Butler AC (2011) The critical role of retrieval practice in long-term retention. Trends Cogn Sci 15(1):20-27.

83. Buchsbaum BR, Lemire-Rodger S, Fang C, Hervé A (2012) The neural basis of vivid memory is patterned on perception. J Cogn Neurosci 24(9):1867-1883. 


\section{Acknowledgements}

This work was supported by Project Grants from the Canadian Institutes for Health Research to M.D.B. (PJT-173336 and PJT-126003), a Scholar Award from the James S McDonnell Foundation to M.D.B., a Connaught Innovation Award to M.D.B., and a Centre for Aging \& Brain Health Innovation (CABHI) Researcher Clinician Partnership Program Grant to M.D.B. We are grateful to Miranda Chang, Alana Changoor, Haleema Khan, Grace Manalili, Arya Rahbarnia, Ashmita Singh, My An Tran, and Shamini Vijaya Kumar for their contributions to recruitment, data collection, and transcription. We thank Celia Fidalgo for assistance with statistical analyses.

\section{Conflict of Interests Statement}

C.B.M, B.H., R.N.N., A.X., C.J.H., and M.D.B. own shares in Dynamic Memory Solutions Inc., a company focused on developing digital tools to improve memory. The University of Toronto holds the ownership rights to the HippoCamera technology used to conduct the research described herein, but has given Dynamic Memory Solutions the rights to commercialize. No person, nor organization received any financial remuneration for the use of the HippoCamera application in these studies. At the time of publication, this is a research-dedicated application that we will make available to other memory scientists at no charge. 


\section{Supplementary Information}

\section{A smartphone intervention that enhances real-world memory and promotes differentiation of hippocampal activity in older adults}

Chris B. Martin, Bryan Hong, Rachel N. Newsome, Katarina Savel, Melissa E. Meade, Andrew Xia, Christopher J. Honey, Morgan D. Barense

\section{Table of Contents}

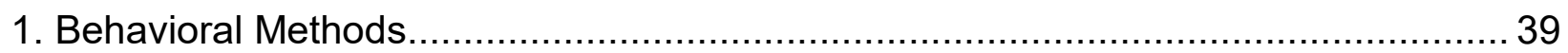

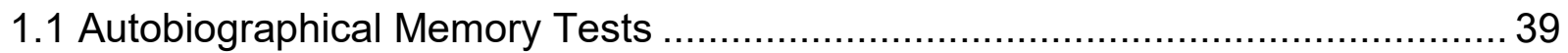

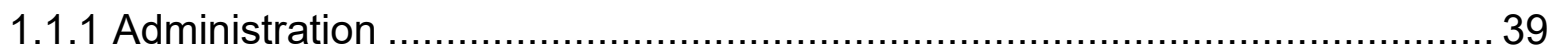

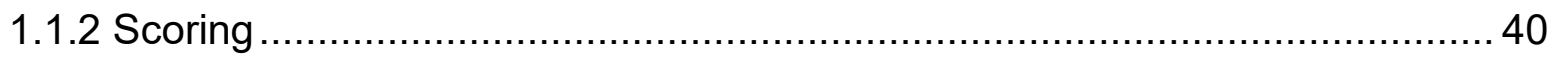

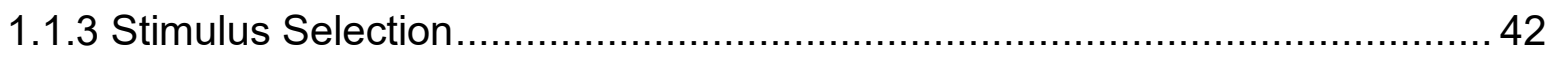

1.2 Linear Mixed Modeling with Autobiographical Memory Test Data ...................... 43

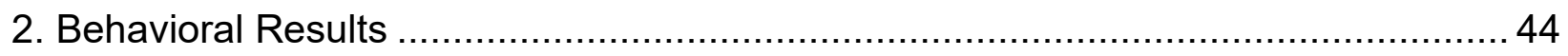

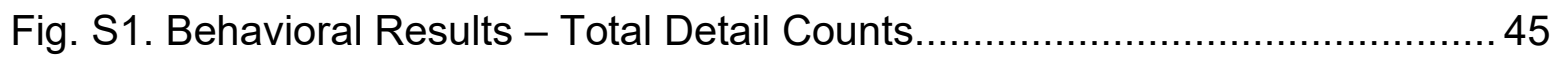

2.1 Autobiographical Memory Test: Total External Details ..................................... 46

2.2 Autobiographical Memory Test: In-Cue Details ............................................ 46

Table S1. Fit statistics and fixed-effects parameters for best fitting models for Internal, External, and In-Cue detail counts. .................................................... 47

2.3 Autobiographical Memory Test: Internal Details by Subtype .............................. 49

Fig. S2. Behavioral Results - Internal Details by Subtype. ................................51

Table S2. Fit statistics and fixed-effects parameters for best fitting models for

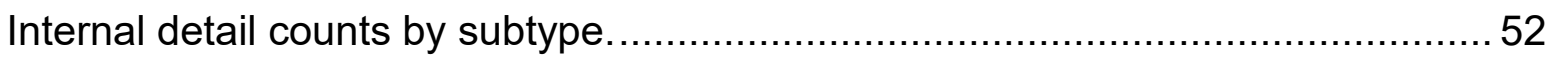

Table S3. Intraclass correlations for intercept-only models of Internal and External

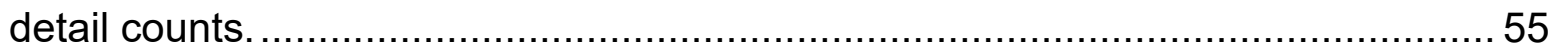

2.4 Autobiographical Memory Test External Details by Subtype ........................... 56

Fig. S3. Behavioral Results - External Details by Subtype................................. 59

Table S4. Fit statistics and fixed-effects parameters for best fitting models for

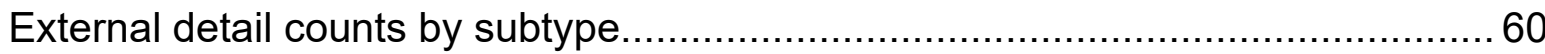

2.5 Autobiographical Memory Test Recall Density ................................................ 64

Table S5. Fit statistics and fixed-effects parameters for best fitting models for

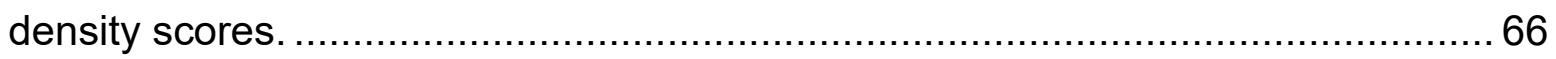

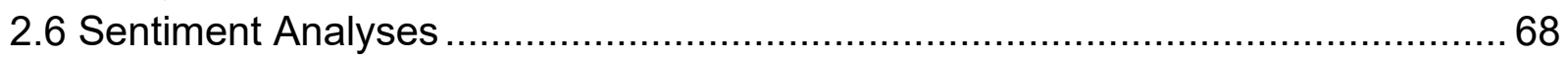

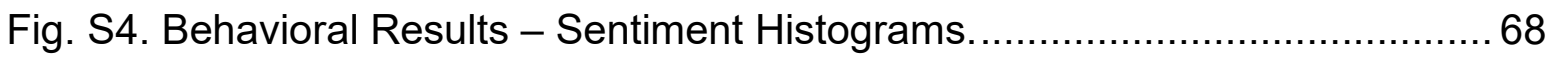

3. fMRI Methods

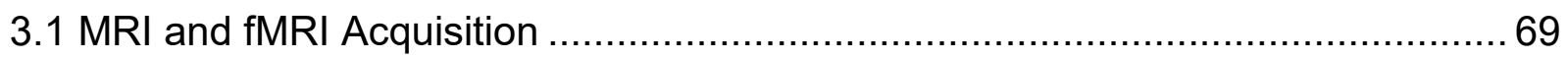

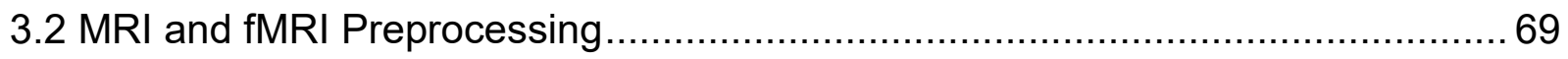

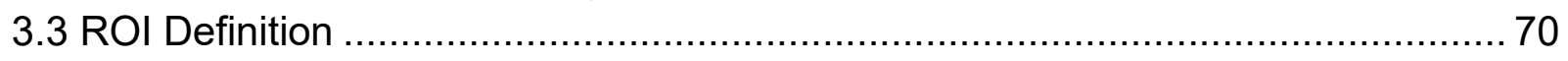




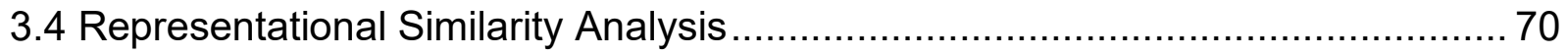

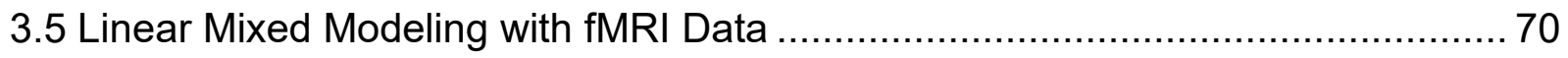

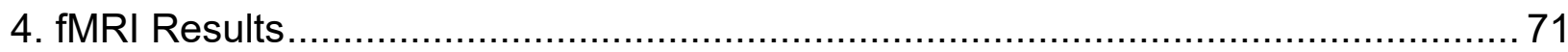

4.1 Pattern Differentiation in the Anterior versus Posterior Hippocampus ................. 71

Fig. S5. Differentiation of Anterior and Posterior Hippocampus Activity ................ 73

4.2 Pattern Differentiation Beyond the Hippocampus ............................................ 73

Fig. S6. Pattern differentiation beyond the hippocampus..................................... 75

Table S6. Fit statistics and fixed-effects parameters for best fitting models of fMRI differentiation by region of interest ............................................................. 76

Table S7. Intraclass correlations for intercept-only models of fMRI differentiation by

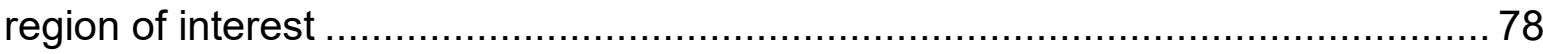

4.3 Relationship Between Pattern Differentiation in the Hippocampus and Recall of

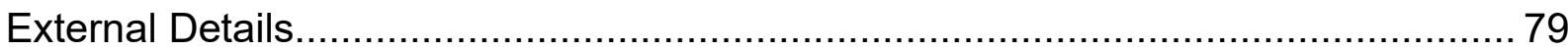

Fig. S7. Correlation Between Differentiation Scores in the Hippocampus and

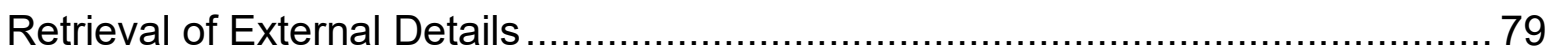

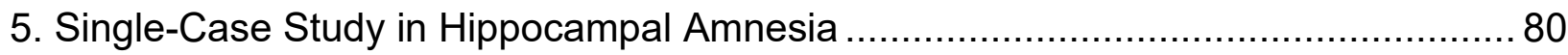

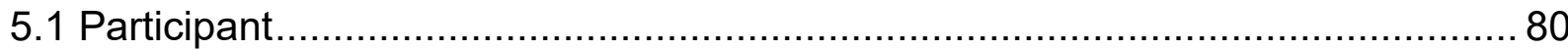

Fig. S8. T1-Weighted MR Image of L.P.'s Bilateral Medial Temporal Lobe Lesion. 80

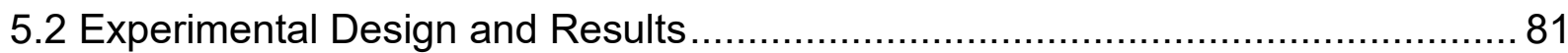

Fig. S9. HippoCamera Replay Improved Recall of Internal Details in LP.............. 82

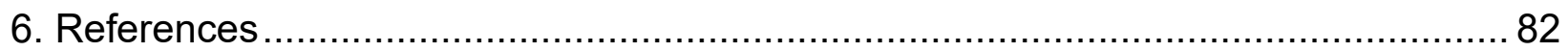

\section{Behavioral Methods}

\subsection{Autobiographical Memory Tests}

\subsubsection{Administration}

We assessed memory performance in Experiment 1 and Experiment 2 using an adapted version of the autobiographical interview (AI) (1). Typical administration of the Al involves asking participants to verbally describe their memory for self-selected events from five different life periods. Following a free-recall response in the typical Al, participants respond to a general probe intended to encourage retrieval of additional information without providing specific guidance. They are then asked to respond to a series of specific probes designed to elicit further detail corresponding to events, time, time integration, place, sensory information, emotions, and thoughts. In our adapted version of the Al, participants were instructed to recall events for which they had previously recorded an autobiographical cue. Memory for each event was tested by first 
having the participant view the event-specific cue and then provide a verbal description of what they remembered based on the following instructions:

"Please tell me as much as you can remember about the event that was just cued. Try to restrict your description to the specific event that was the subject of the recording. It is important that your description goes beyond the contents of the video and verbal description that you recorded. In other words, do not simply narrate the video, tell me about the entirety of the event."

The length of event-specific verbal descriptions elicited by this approach varied across events and participants. Early testing cued-recall responses ranged from 15 seconds to 20 minutes per event. Given that we were probing memory for up to 40 distinct events, rather than five as is typical in the $\mathrm{Al}$, we did not consistently administer general or specific probes. As such, all data described in this report reflect details described in initial cued-recall responses.

\subsubsection{Scoring}

All raters successfully completed the Al training protocol (1). Cued-recall responses were quantified using an adapted version of the Al scoring protocol that specifies criteria for distinguishing between Internal and External details, as well as subtypes of Internal and External details (1,2). Our adaptation further quantified In-Cue details.

Internal Detail Subtypes

- Internal Event details: recall of information that is episodic in nature and pertains to the cued event. This includes happenings, individuals present, weather conditions, physical/emotional actions, or reactions in others.

- Internal Time details: year, season, month, day of week, time of day

- Internal Place details: localization of an event including the city, street, building, room, part of room.

- Internal Perceptual details: auditory, olfactory, tactile, taste, visual and visual details, body position, duration. 
- Internal Thought/Emotion details: emotional state, thoughts, implications.

\section{External Detail Subtypes}

- External Event details: recall of information that is episodic in nature but pertains to non-target events.

- General Semantic details: culturally shared knowledge of facts, public events, people, and concepts.

- Personal Semantic details: semantic knowledge of one's personal past, which is further divided into three subtypes.

- Autobiographical Facts: basic units of knowledge about one's personal past, such as the name of the street on which you lived as a child, or your dog's name.

- Self-Knowledge: awareness of one's disposition and preferences, such as claiming that you are hot tempered or noting that you dislike spicy food.

- Repeated Events: descriptions of features that are common to multiple instances of an episode, such as mentioning that you and a friend always order the same cobbler for dessert when you get together for dinner on Saturdays.

- Repeated Details: information provided multiple times while describing memory for a specific event.

- Other: utterances that could not otherwise be scored as Internal or any of the above External subtypes (e.g., "Give me a moment to think about this", "I don't remember this event very well").

In-Cue Details

- In-Cue details refer to verbal descriptions that capture information that was apparent in the cue from all trial-specific detail counts. These details were omitted from Internal and External detail counts. For example, if a cue included video footage of a dinner partner who was wearing a red dress and eating chicken, any 
mention of the red dress or specific comments about the chicken dish were not counted toward Internal and External detail counts. This strategy ensured that our dependent measure reflected episodic reliving rather than mere recall of content from the multi-modal cue.

\subsubsection{Stimulus Selection}

Anecdotally, the quality of cues varied within and across participants and experiments. Some cues corresponded to interesting and complex events (e.g., attending an outdoor concert at a summer festival), whereas others captured more mundane moments from daily life (e.g., preparing lunch). On average, participants in Experiment 1 recorded a total of 66.9 cues (33.8 were randomly assigned to the Replayed condition and 33.1 to the Baseline condition) over their 14-day use period. Participants in Experiment 2 recorded a total of 66.75 cues (33.9 in the Replayed condition and 32.9 in the Baseline condition) over their 70-day use period. Because of the extensive nature of our autobiographical memory tests, we selected up to 40 of these cues for the purpose of constructing our behavioral and fMRI assessments (up to 20 Replayed and up to 20 Baseline). In some cases, fewer than 40 cues were selected based on three criteria. First, across conditions we aimed to match the events for which we were testing memory at the level of event frequency, event significance, and memory age. Second, we selected cues that sampled broadly from the range of recorded behaviors and events to ensure that we were probing memory for experiences that were representative of our participants' lives. Cues were excluded from testing if they received low significance ratings (i.e., a rating of 1 or 2 out of 5). Third, in Experiment 1, multiple cues that obviously corresponded to the same event (e.g., a birthday party with one cue of the cake and another of presents being opened), but were randomly assigned to different conditions (i.e., one cue was Replayed whereas the other was Baseline), were not tested in either the behavioral or fMRI experiments.

Using this approach, we successfully matched Experiment 1 events across the Replayed and Baseline conditions at the level of event frequency ( $M_{\text {Replayed }}=2.52$ on a 5-point scale where 1 corresponds to unique events and 5 corresponds to daily events, $M_{\text {Baseline }}=2.60$; paired $\left.t(21)=0.72, P=.48\right)$ and event significance $\left(M_{\text {Replayed }}=3.13\right.$ on a 
5-point scale where 1 corresponds to the least significant events and 5 corresponds to the most significant events, $M_{\text {Baseline }}=3.13$; paired $\left.t(21)=0.01, P=.99\right)$. There was a statistically significant difference between Replayed and Baseline events at the level of memory age, such that Replayed memories were older (Early Mage: Replayed $=4.3$ days \pm .16 SEM, Baseline $=4.0$ days \pm .20 SEM, paired $t(20)=2.49, P=.02, d=0.54$; Delayed Mage: Replayed $=123.8$ days \pm 4.92 SEM, Baseline $=123.4$ days \pm 4.90 SEM, paired $t(18)=2.17, \mathrm{P}=.04, d=0.50)$. We note, however, that this difference was rather small in practical terms and that its direction (Replayed older than Baseline) works against our hypotheses regarding enhanced memory in the Replayed condition.

In Experiment 2, Replayed and Baseline memories were matched at the levels of event significance ( $M_{\text {Replayed }}=3.32$ on a 5-point scale, $M_{\text {Baseline }}=3.24$; paired $t(11)=1.08$, $\mathrm{P}=.30$ ), event frequency ( $\mathrm{M}_{\text {Replayed }}=2.52$ on a 5-point scale, $\mathrm{M}_{\text {Baseline }}=2.28$; paired $t(11)$ $=2.01, \mathrm{P}=.06$ ), and memory age (Early Mage: Replayed $=43.35$ days $\pm 2.31 \mathrm{SEM}$, Baseline $=44.98$ days \pm 1.93 SEM, paired $t(11)=0.54, P=0.59$; Delayed Mage: Replayed $=144.55$ days \pm 4.51 SEM, Baseline $=146.16$ days $\pm 4.11 \mathrm{SEM}$, paired $t(11)=0.26, \mathrm{P}=$ $0.79)$.

\subsection{Linear Mixed Modeling with Autobiographical Memory Test Data}

Detail counts from the autobiographical memory tests were analyzed using multilevel modeling in R 4.1.0 (R Core Team, 2021) with the Ime4 package (3). The performance package was used to obtain intraclass correlations (ICC) and both conditional and marginal coefficients of determination $\left(R^{2} C\right.$ and $R^{2} M$, respectively) to determine model fits $(4,5)$. To test hypotheses related to autobiographical memory test detail counts, we used 2-level multilevel generalized Poisson models with individual trials nested within participants. Poisson models were used to best account for the count-based nature of the number of details recollected (6). For both Experiment 1 (2-Week Intervention) and Experiment 2 (10-Week Intervention), individual models were specified for Internal details, External details, and In-Cue details to obtain separate estimates for each detail type. Multilevel modeling was appropriate given the degree of variance explained by individual participants in Experiment 1 and Experiment 2 (Table S1). For all models, we first fit the model with the maximal random effects structure (7). To investigate 
the effect of condition and test time point, this entailed estimating fixed effects for condition (Replayed vs. Baseline), test time point (Early vs. Delayed), and their interaction, as well as a random intercept estimated for each participant and a random slope estimated for each fixed effect. condition and test session were effect coded (condition: Baseline $=-1$, Replayed $=1$; test time point: Early $=-1$, Delayed $=1$ ). In the situation that the maximal model failed to converge due to overparameterization, we employed a backward-selection heuristic (8). To probe for any significant interactions, simple effects tests were conducted with the Tukey adjustment for pairwise comparisons using the emmeans package (9)—adjusted $P$-values are reported for simple effects. To compare the total number of details recalled across both experiments, the maximal model predicting the total number of details recalled on a trial with a fixed effect for Experiment (Experiment 1 vs Experiment 2), a random slope for Experiment, and a random intercept for each participant was first specified-Experiment was effect coded (Experiment 1 = 1 , Experiment $2=1$ ). Model selection was performed in the same fashion as described above. All models were estimated with an unstructured covariance matrix.

\section{Behavioral Results}

Overall Internal, External, and In-Cue detail counts are plotted in Fig. S1 to allow for direct comparison. Model fits for overall details of all types are reported in Table S1. Statistical analyses pertaining to overall Internal detail counts are reported in the main text of our manuscript (Fig. 2). 


\section{Experiment 1 (2-Week Intervention)}

\section{Early}

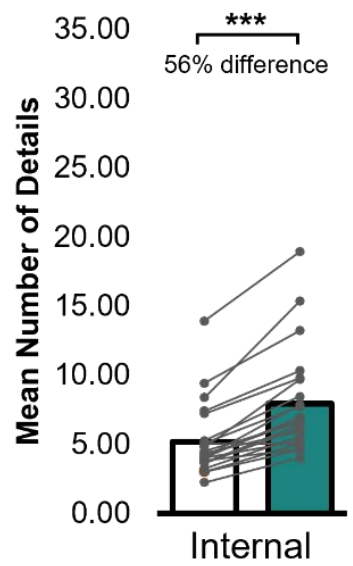

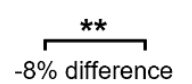
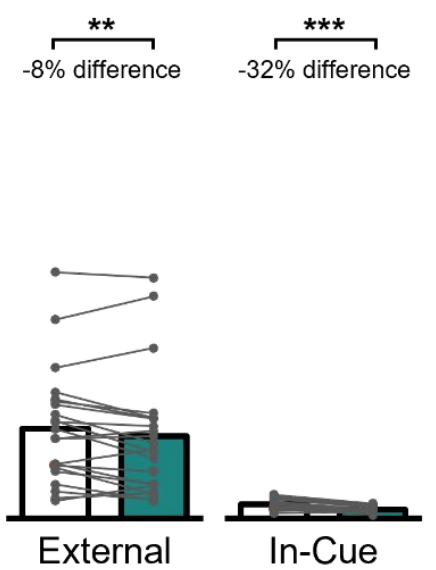

\section{Delayed}

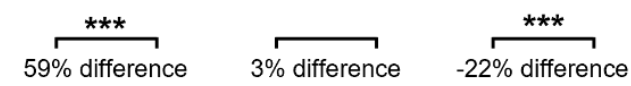

\section{Experiment 2 (10-Week Intervention)}
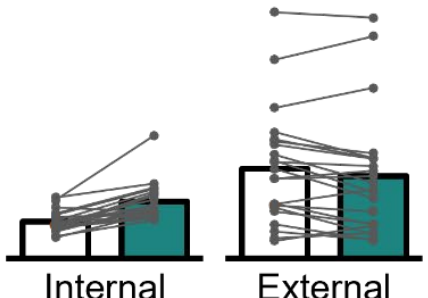

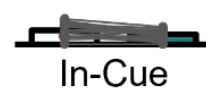

\section{Early}

35.00

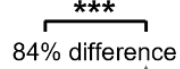

$84 \%$ difference

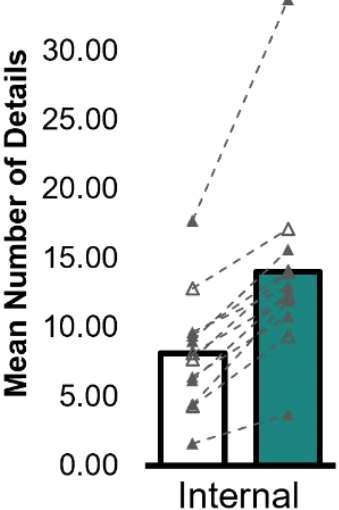

$-8 \%$ difference
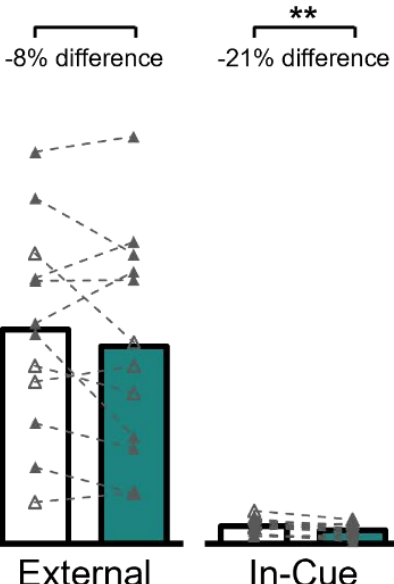

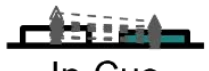

In-Cue

\section{Delayed}
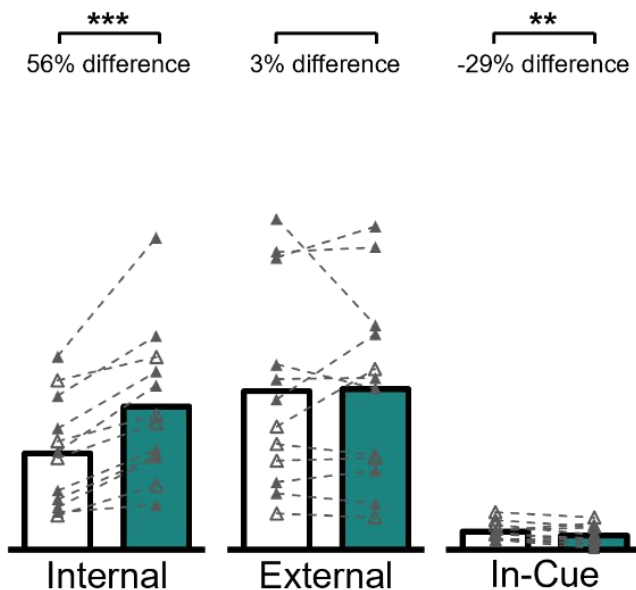

External

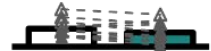

In-Cue

\section{$\square$ Baseline $\square$ Replayed}

Fig. S1. Behavioral Results - Total Detail Counts. Mean number of Internal, External, and In-Cue details for Experiment 1 and Experiment 2. Early testing corresponds to behavioral performance measured during (Experiment 1) and shortly after (Experiment 2) HippoCamera use. Delayed testing corresponds to behavioral performance after a 3-month delay, during which time participants did not have access to their memory cues. Open triangles denote Experiment 2 participants who failed the Montreal Cognitive Assessment. ${ }^{*}<.05,{ }^{* *}<.01,{ }^{* * *}<.001$. 


\subsection{Autobiographical Memory Test: Total External Details}

We performed exploratory analyses focused on total External details (Fig. S1, Table S1-2). For Experiment 1, we found a significant main effect of condition (Replayed vs. Baseline: $b=-0.0335, S E=0.0171, z=-1.964, P=.049$ ) but not test time point (Early vs. Delayed: $b=-0.0811, S E=0.0427, z=-1.899, P=.057$ ). There was also a significant interaction between condition and test time point $(b=0.0342, \mathrm{SE}=0.0172, \mathrm{z}=1.986, P$ $=.047)$. Comparison of simple slopes revealed that participants provided significantly more External details for Baseline events at Early testing test sessions $(b=-0.135$, SE $=$ $0.0520, z=-2.603, p=.0092)$, but not Delayed testing test sessions $(b=0.00139, \mathrm{SE}=$ $0.0447, z=0.031, p=.975)$. In contrast to Experiment 1 , the main effect of condition was not significant in Experiment $2(b=-0.0259, \mathrm{SE}=0.0189, z=-1.373, p=.170)$, but it was for test time point $(b=-0.146, \mathrm{SE}=0.0355, z=-4.104, p<.001)$. Participants provided more External details at Early testing than they did at Delayed testing. The interaction term between condition and test time point was not significant $(b=0.0322$, SE $=0.0301$, $z=1.069, p=.285$ ). Overall, these data suggest that HippoCamera replay specifically enhances recall of Internal details from everyday experiences.

\subsection{Autobiographical Memory Test: In-Cue Details}

In-Cue details were common but represented a relatively small proportion of total details recalled (Fig. S1, Table S1-2). Analysis of In-Cue details revealed converging results across Experiment 1 and Experiment 2. In both cases, we found a main effect of condition (Replayed vs. Baseline; Experiment 1: $b=-0.207$, SE $=0.0295, z=-7.024, p$ $<.001$; Experiment 2: $b=-0.194, \mathrm{SE}=0.0624, z=-3.111, \mathrm{P}=.00186$ ) that reflected fewer details in the Replayed condition than in the Baseline condition. The main effect of test time point was not significant (Early vs. Delayed; Experiment 1: $b=-0.00115$, SE $=0.0381, z=-0.030, p=.976$; Experiment 2: $b=0.0269$, SE $=0.0307, z=0.876, p=$ .381). There were also no significant interactions between condition and test time point (Experiment 1: $b=0.0133, \mathrm{SE}=0.0295, z=0.450, p=.652 ; b=0.00563, \mathrm{SE}=0.0307$, $z=0.183, p=.855)$. These results suggest that participants relied less on information reflected in their cues and more on elaborative retrieval processes following repeated replay with HippoCamera. 


\section{ENHANCING REAL-WORLD MEMORY}

Table S1. Fit statistics and fixed-effects parameters for best fitting models for Internal, External, and In-Cue detail counts.

\begin{tabular}{|c|c|c|c|c|c|c|c|c|c|}
\hline Experiment & Detail Type & Model Formula & $\mathbf{R}^{2} \mathrm{C}$ & $\mathbf{R}^{2}{ }_{M}$ & Fixed Effect & Estimate (b) & SE & $\mathbf{z}$ & $P$ \\
\hline \multirow[t]{4}{*}{ Experiment 1} & Internal & $\begin{array}{l}\sim \text { condition } \times \text { test time point }+(\text { condition }+ \text { test } \\
\text { time point | participant })\end{array}$ & .493 & .264 & intercept & 1.477 & 0.0649 & 22.757 & $<2 \mathrm{e}-16^{* * *}$ \\
\hline & & & & & condition & 0.212 & 0.0183 & 11.593 & $<2 \mathrm{e}-16^{* * *}$ \\
\hline & & & & & test time point & -0.292 & 0.0336 & -8.676 & $<2 \mathrm{e}-16^{* * *}$ \\
\hline & & & & & $\begin{array}{l}\text { condition } \times \text { test time } \\
\text { point }\end{array}$ & -0.00533 & 0.0134 & -0.397 & .691 \\
\hline \multirow[t]{4}{*}{ Experiment 1} & External & $\begin{array}{l}\sim \text { condition } \times \text { test time point }+(\text { condition } \times \text { test } \\
\text { time point | participant })\end{array}$ & .650 & .015 & intercept & 1.525 & 0.125 & 12.201 & $<2 \mathrm{e}-16^{* * *}$ \\
\hline & & & & & condition & -0.0335 & 0.0171 & -1.964 & .0575 \\
\hline & & & & & test time point & -0.0811 & 0.0427 & -1.899 & .0495 * \\
\hline & & & & & $\begin{array}{l}\text { condition } \times \text { test time } \\
\text { point }\end{array}$ & 0.0342 & 0.0172 & 1.986 & .0471 * \\
\hline \multirow[t]{4}{*}{ Experiment 1} & In-Cue & $\begin{array}{l}\sim \text { condition } \times \text { test time point }+ \text { (test time point } \mid \\
\text { participant) }\end{array}$ & .158 & .045 & intercept & -0.249 & 0.0754 & -3.297 & $.000976^{* \star *}$ \\
\hline & & & & & condition & -0.207 & 0.0295 & -7.024 & $2.15 \mathrm{e}-12^{* * *}$ \\
\hline & & & & & test time point & -0.00115 & 0.0381 & -0.030 & .976 \\
\hline & & & & & $\begin{array}{l}\text { condition } \times \text { test time } \\
\text { point }\end{array}$ & 0.0133 & 0.0295 & 0.450 & .653 \\
\hline \multirow[t]{4}{*}{ Experiment 2} & Internal & $\begin{array}{l}\sim \text { condition } \times \text { test time point }+ \text { (condition } \times \text { test } \\
\text { time point | participant })\end{array}$ & .828 & .162 & intercept & 2.183 & 0.144 & 15.114 & $<2 \mathrm{e}-16^{* * *}$ \\
\hline & & & & & condition & 0.222 & 0.0264 & 8.407 & $<2 \mathrm{e}-16^{* * *}$ \\
\hline & & & & & test time point & -0.120 & 0.0312 & -3.840 & $0.000123^{* * *}$ \\
\hline & & & & & $\begin{array}{l}\text { condition } \times \text { test time } \\
\text { point }\end{array}$ & -0.0369 & 0.0142 & -2.607 & $0.009143^{* *}$ \\
\hline
\end{tabular}




\section{ENHANCING REAL-WORLD MEMORY}

\begin{tabular}{|c|c|c|c|c|c|c|c|c|c|}
\hline Experiment & Detail Type & Model Formula & $\mathbf{R}^{2} \mathrm{C}$ & $\mathbf{R}^{2}{ }_{M}$ & Fixed Effect & Estimate (b) & SE & z & $P$ \\
\hline \multirow[t]{4}{*}{ Experiment 2} & External & $\begin{array}{l}\sim \text { condition } \times \text { test time point }+ \text { (condition } \times \text { test } \\
\text { time point | participant) }\end{array}$ & .893 & .041 & intercept & 2.434 & 0.185 & 13.133 & $<2 \mathrm{e}-16^{* \star *}$ \\
\hline & & & & & condition & -0.0278 & 0.0181 & -1.536 & .124 \\
\hline & & & & & test time point & -0.138 & 0.0323 & -4.288 & $1.8 \mathrm{e}-05^{* * *}$ \\
\hline & & & & & $\begin{array}{l}\text { condition } \times \text { test time } \\
\text { point }\end{array}$ & 0.0314 & 0.0289 & 1.087 & .277 \\
\hline \multirow[t]{4}{*}{ Experiment 2} & In-Cue & $\sim$ condition $\times$ test time point $+($ condition $\mid$ participant $)$ & .366 & .043 & intercept & -0.0173 & 0.151 & -0.114 & .909 \\
\hline & & & & & condition & -0.194 & 0.0624 & -3.111 & .00186 ** \\
\hline & & & & & test time point & 0.0269 & 0.0307 & 0.876 & .381 \\
\hline & & & & & $\begin{array}{l}\text { condition } \times \text { test time } \\
\text { point }\end{array}$ & 0.00563 & 0.0307 & 0.183 & .855 \\
\hline \multirow[t]{2}{*}{ N/A } & Overall & $\sim$ intervention length $+(1 \mid$ participant $)$ & .815 & .336 & intercept & 2.667 & 0.0792 & 33.681 & $<2 \mathrm{e}-16^{* * *}$ \\
\hline & & & & & condition & 0.376 & 0.0792 & 4.751 & $2.02 \mathrm{e}-06^{* * *}$ \\
\hline
\end{tabular}

Legend: $\mathrm{R}^{2} \mathrm{C}=$ conditional coefficient of determination, $\mathrm{R}^{2} \mathrm{M}$ : marginal coefficient of determination, SE: standard error, ${ }^{* *} P<.01,{ }^{* \star *} P<.001$. Note: models specified here are multilevel generalized Poisson models to best account for the count-based nature of the number of details recollected. 


\subsection{Autobiographical Memory Test: Internal Details by Subtype}

Our primary behavioral analyses revealed that HippoCamera replay selectively enhanced recall of Internal details. We next examined whether this effect reflected an increase in all subtypes of Internal details. Consistent with previous evidence from older adults (1), most Internal details were of the Event detail subtype (57\% of all Internal details were Event details in both experiments). The remaining details were roughly evenly distributed among Place, Perceptual, and Thought/Emotion details. Time details were least frequently described. The most consistent result across detail types was a main effect of condition (Replayed > Baseline; Fig. S2; Table S2-3).

Internal Event: We found a significant main effect of condition (Replayed vs. Baseline) in Experiment $1(b=0.224, S E=0.0257, z=8.726, p<.001)$ and Experiment $2(b=$ $0.276, \mathrm{SE}=0.0420, z=6.577, p<.001)$. There was also a significant main effect of test time point (Early vs. Delayed) in Experiment $1(b=-0.294, S E=0.0405, z=-7.267, p<$ $.001)$ and Experiment $2(b=-0.120, S E=0.0391, z=-3.079, p=.00208)$. There were no significant interactions between condition and test time point in either experiment (Experiment 1: $b=-0.0177, \mathrm{SE}=0.0181, \mathrm{z}=-0.976, \mathrm{p}=.329$; Experiment 2: $\mathrm{b}=-0.0309$, $\mathrm{SE}=0.0200, z=-1.549, p=.121)$. In sum, participants recalled more Internal Event details for Replayed events than Baseline events, and at Early than Delayed testing.

Internal Time: We found a significant main effect of condition (Replayed vs. Baseline) in Experiment $1(\mathrm{~b}=0.137, \mathrm{SE}=0.0448, \mathrm{z}=3.059, \mathrm{p}=.00222)$ and Experiment $2(\mathrm{~b}=$ $0.236, S E=0.0631, z=3.434, p<.001)$. There was also a significant main effect of test time point (Early vs. Delayed) in Experiment $1(b=-0.379, S E=0.0674, z=-5.634, p<$ $.001)$ and Experiment $2(b=-0.233, S E=0.0538, z=-4.331, p<.001)$. There were no significant interactions between condition and test time point in either experiment (Experiment 1: $b=0.00328, S E=0.0448, z=0.073, p=.942$; Experiment 2: $b=0.0336$, $\mathrm{SE}=0.0538, z=0.625, p=.532$ ). In sum, participants recalled more Internal Time details for Replayed events than Baseline events, and at Early than Delayed testing.

Internal Thought/Emotion: We found a significant main effect of condition (Replayed vs. Baseline) in Experiment $1(b=0.139$, SE $=0.0501, z=2.764, p=.00571)$ and Experiment $2(b=0.249, \mathrm{SE}=0.0423, z=5.882, p<.001)$. There was also a significant 
main effect of test time point (Early vs. Delayed) in Experiment $1(b=-0.326, \mathrm{SE}=0.0434$, $z=-7.520, p<.001)$ and Experiment $2(b=-0.128, \mathrm{SE}=0.0484, z=-2.634, p=.00843)$. There were no significant interactions between condition and test time point in either experiment (Experiment 1: $b=-0.0492$, SE $=0.0331, z=-1.483, p=.138$; Experiment 2: $b=-0.0421, \mathrm{SE}=0.0285, z=-1.481, p=.139)$. In sum, participants recalled more Internal Thought/Emotion details for Replayed events than Baseline events, and at Early than Delayed testing.

Internal Place: We found a significant main effect of condition (Replayed vs. Baseline) in Experiment $1(b=0.363, \mathrm{SE}=0.0409, z=8.885, p<.001)$ and Experiment $2(b=$ $0.227, \mathrm{SE}=0.0363, z=6.258, p<.001)$. There were no significant main effects of test time point (Early vs. Delayed) in either experiment (Experiment 1: $b=-0.0755$, SE $=$ $0.0532, z=-1.420, p=.156$; Experiment 2: $b=-0.0417, \mathrm{SE}=0.0489, z=-0.854, p=$ .393). There were no significant interactions between condition and test time point in either experiment (Experiment 1: $b=-0.0154, \mathrm{SE}=0.0409, z=-0.376, p=.707$; Experiment 2: $b=-0.0378, \mathrm{SE}=0.0363, z=-1.040, p=.298$ ). In sum, participants recalled more Internal Place details for Replayed events than Baseline events.

Internal Perceptual: We found a significant main effect of condition (Replayed vs. Baseline $)$ in Experiment $1(b=0.179, \mathrm{SE}=0.0569, z=3.137, p=.00170)$ and Experiment $2(b=0.212, S E=0.0269, z=7.865, p<.001)$. There was also a significant main effect of test time point (Early vs. Delayed) in Experiment $1(b=-0.331$, $S E=0.0612, z=-5.404$, $p<.001)$ and Experiment $2(b=-0.148, S E=0.0479, z=-3.077, p=.00209)$. The interaction between condition and test time point was not significant in Experiment $1 \mathrm{~b}=$ 0.0816, SE $=0.0553, z=1.474, p=.141)$ but was in Experiment $2(b=-0.0745, S E=$ $0.0269, z=-2.766, p=.00567)$. The significant interaction in Experiment 2 was driven by the fact that the increase in Internal Perceptual details for Replayed events relative to Baseline events was greater at Early testing $(b=0.573, S E=0.0720, z=7.956, p<.001)$ than it was at Delayed testing $(b=0.275, S E=0.0802, z=3.426, p<.001)$. 


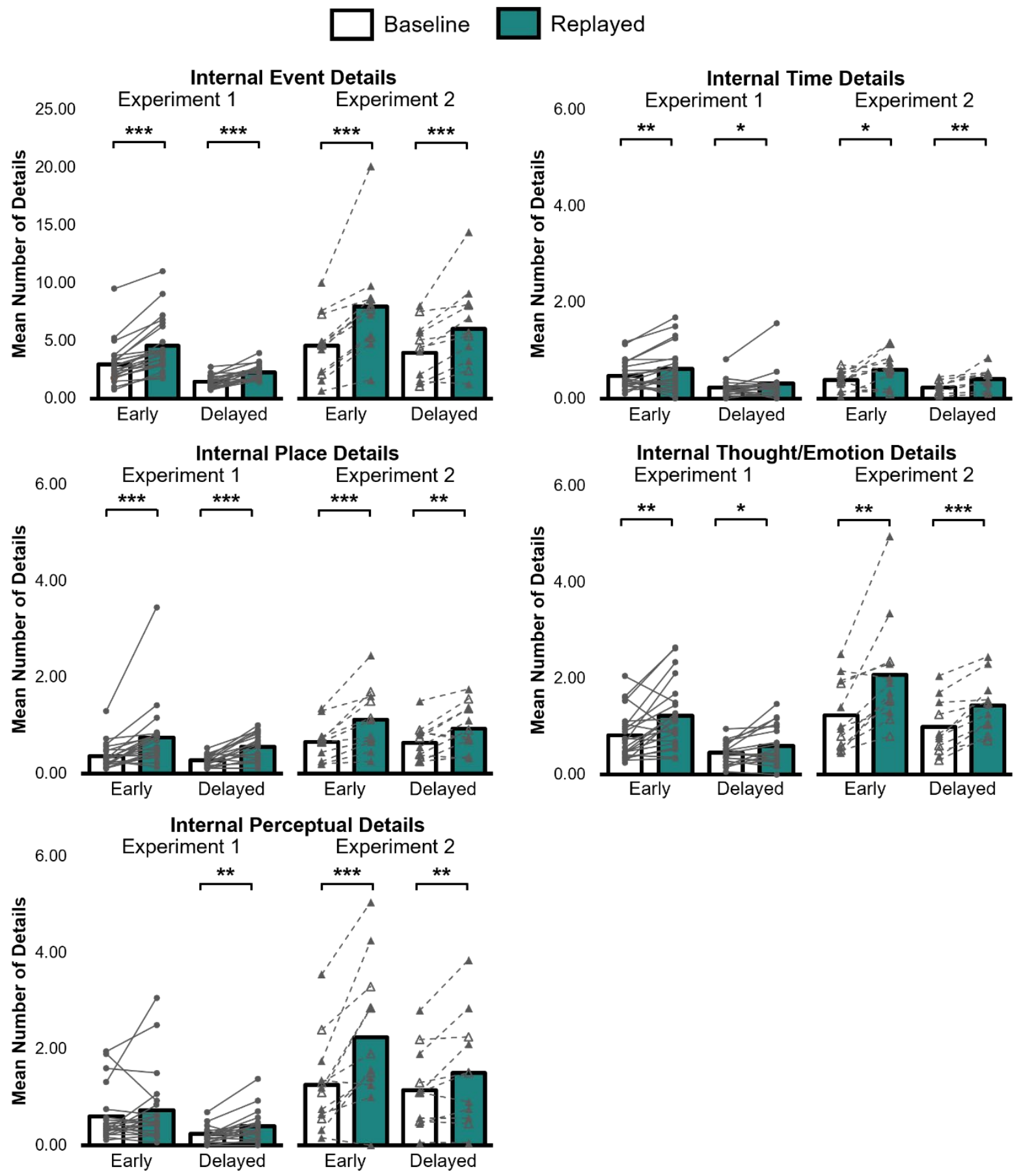

Fig. S2. Behavioral Results - Internal Details by Subtype. Mean number of Internal details by subtype for Experiment 1 and Experiment 2. Early testing corresponds to behavioral performance measured during (Experiment 1) and shortly after (Experiment 2) HippoCamera use. Delayed testing corresponds to behavioral performance after a 3-month delay, during which time participants did not have access to their memory cues. Open triangles denote Experiment 2 participants who failed the Montreal Cognitive Assessment. ${ }^{*}<.05,{ }^{* *}<.01,{ }^{* * *}<.001$. 


\section{ENHANCING REAL-WORLD MEMORY}

Table S2. Fit statistics and fixed-effects parameters for best fitting models for Internal detail counts by subtype.

\begin{tabular}{|c|c|c|c|c|c|c|c|c|c|}
\hline Experiment & Detail Type & Model Formula & $\mathbf{R}^{2}{ }_{\mathrm{C}}$ & $\mathbf{R}^{2}{ }_{M}$ & Fixed Effect & Estimate (b) & SE & z & $P$ \\
\hline \multirow[t]{4}{*}{ Experiment 1} & Event & $\begin{array}{l}\sim \text { condition } \times \text { test time point }+ \text { (condition }+ \\
\text { test time point | participant) }\end{array}$ & .423 & .199 & intercept & 0.886 & 0.0749 & 11.835 & $<2 \mathrm{e}-16^{* * *}$ \\
\hline & & & & & condition & 0.224 & 0.0257 & 8.726 & $<2 \mathrm{e}-16^{* \star *}$ \\
\hline & & & & & test time point & -0.294 & 0.0405 & -7.267 & $3.69 \mathrm{e}-13^{* * *}$ \\
\hline & & & & & $\begin{array}{l}\text { condition } \times \text { test } \\
\text { time point }\end{array}$ & -0.0177 & 0.0181 & -0.976 & .329 \\
\hline \multirow[t]{4}{*}{ Experiment 1} & Time & $\begin{array}{l}\sim \text { condition } \times \text { test time point }+ \text { (test time } \\
\text { point I participant) }\end{array}$ & .257 & .085 & intercept & -1.157 & 0.129 & -8.975 & $<2 \mathrm{e}-16^{* * *}$ \\
\hline & & & & & condition & 0.137 & 0.0448 & 3.059 & .00222 ** \\
\hline & & & & & test time point & -0.379 & 0.0674 & -5.634 & $1.76 \mathrm{e}-08$ *** \\
\hline & & & & & $\begin{array}{l}\text { condition } \times \text { test } \\
\text { time point }\end{array}$ & 0.00328 & 0.0448 & 0.073 & .942 \\
\hline \multirow[t]{4}{*}{ Experiment 1} & Place & $\begin{array}{l}\sim \text { condition } \times \text { test time point }+ \text { (test time } \\
\text { point | participant) }\end{array}$ & .228 & .087 & intercept & -0.897 & 0.107 & -8.390 & $<2 \mathrm{e}-16^{* * *}$ \\
\hline & & & & & condition & 0.363 & 0.0409 & 8.885 & $<2 \mathrm{e}-16^{* \star *}$ \\
\hline & & & & & test time point & -0.0755 & 0.0532 & -1.420 & .156 \\
\hline & & & & & $\begin{array}{l}\text { condition } \times \text { test } \\
\text { time point }\end{array}$ & -0.0154 & 0.0409 & -0.376 & .707 \\
\hline \multirow[t]{4}{*}{ Experiment 1} & Perceptual & $\begin{array}{l}\sim \text { condition } \times \text { test time point }+ \text { (condition } \times \\
\text { test time point I participant) }\end{array}$ & .313 & .067 & intercept & -1.040 & 0.158 & -6.597 & $4.20 \mathrm{e}-11$ *** \\
\hline & & & & & condition & 0.179 & 0.0569 & 3.137 & .00170 ** \\
\hline & & & & & test time point & -0.331 & 0.0612 & -5.404 & $6.52 \mathrm{e}-08^{* * *}$ \\
\hline & & & & & $\begin{array}{l}\text { condition } \times \text { test } \\
\text { time point }\end{array}$ & 0.0816 & 0.0553 & 1.474 & .141 \\
\hline
\end{tabular}




\section{ENHANCING REAL-WORLD MEMORY}

\begin{tabular}{|c|c|c|c|c|c|c|c|c|c|}
\hline Experiment & Detail Type & Model Formula & $\mathbf{R}^{2} \mathrm{c}$ & $\mathbf{R}^{2} \mathrm{M}$ & Fixed Effect & Estimate (b) & SE & $\mathbf{z}$ & $P$ \\
\hline \multirow[t]{4}{*}{ Experiment 1} & Thought/Emotion & $\begin{array}{l}\sim \text { condition } \times \text { test time point }+ \text { (condition }+ \\
\text { test time point } \mid \text { participant })\end{array}$ & .274 & .092 & intercept & -0.445 & 0.106 & -4.177 & $2.95 \mathrm{e}-05^{* * \star}$ \\
\hline & & & & & condition & 0.139 & 0.0501 & 2.764 & .00571 ** \\
\hline & & & & & test time point & -0.326 & 0.0434 & -7.520 & $5.47 e-14^{* * *}$ \\
\hline & & & & & $\begin{array}{l}\text { condition } \times \text { test } \\
\text { time point }\end{array}$ & -0.0492 & 0.0331 & -1.483 & .138 \\
\hline \multirow[t]{4}{*}{ Experiment 2} & Event & $\begin{array}{l}\sim \text { condition } \times \text { test time point }+ \text { (condition } \times \\
\text { test time point | participant) }\end{array}$ & .847 & .151 & intercept & 1.505 & 0.181 & 8.314 & $<2 \mathrm{e}-16^{* * *}$ \\
\hline & & & & & condition & 0.276 & 0.0420 & 6.577 & $4.79 \mathrm{e}-11^{* * \star}$ \\
\hline & & & & & test time point & -0.120 & 0.0391 & -3.079 & $.00208^{* *}$ \\
\hline & & & & & $\begin{array}{l}\text { condition } \times \text { test } \\
\text { time point }\end{array}$ & -0.0309 & 0.0200 & -1.549 & .121 \\
\hline \multirow[t]{4}{*}{ Experiment 2} & Time & $\begin{array}{l}\sim \text { condition } \times \text { test time point }+ \text { (condition I } \\
\text { participant) }\end{array}$ & .188 & .060 & intercept & -1.059 & 0.152 & -6.973 & $3.10 \mathrm{e}-12^{* * *}$ \\
\hline & & & & & condition & 0.236 & 0.0631 & 3.734 & $.000188^{* * *}$ \\
\hline & & & & & test time point & -0.233 & 0.0538 & -4.331 & $1.49 \mathrm{e}-05^{* * *}$ \\
\hline & & & & & $\begin{array}{l}\text { condition } \times \text { test } \\
\text { time point }\end{array}$ & 0.0336 & 0.0538 & 0.625 & .532 \\
\hline \multirow[t]{4}{*}{ Experiment 2} & Place & $\begin{array}{l}\sim \text { condition } \times \text { test time point }+ \text { (test time } \\
\text { point | participant) }\end{array}$ & .277 & .045 & intercept & -0.337 & 0.157 & -2.147 & $.0318^{*}$ \\
\hline & & & & & condition & 0.227 & 0.0363 & 6.258 & $3.89 e-10^{* * *}$ \\
\hline & & & & & test time point & -0.0417 & 0.0489 & -0.854 & .393 \\
\hline & & & & & $\begin{array}{l}\text { condition } \times \text { test } \\
\text { time point }\end{array}$ & -0.0378 & 0.0363 & -1.040 & .298 \\
\hline
\end{tabular}




\section{ENHANCING REAL-WORLD MEMORY}

\begin{tabular}{|c|c|c|c|c|c|c|c|c|c|}
\hline Experiment & Detail Type & Model Formula & $\mathbf{R}^{2} \mathrm{c}$ & $\mathbf{R}^{2}{ }_{M}$ & Fixed Effect & Estimate (b) & SE & z & $P$ \\
\hline \multirow[t]{4}{*}{ Experiment 2} & Perceptual & $\begin{array}{l}\sim \text { condition } \times \text { test time point }+ \text { (test time } \\
\text { point | participant) }\end{array}$ & .555 & .043 & intercept & 0.0959 & 0.270 & 0.355 & .722 \\
\hline & & & & & condition & 0.212 & 0.0269 & 7.865 & $3.70 \mathrm{e}-15^{* \star *}$ \\
\hline & & & & & test time point & -0.148 & 0.0479 & -3.077 & .00209 ** \\
\hline & & & & & $\begin{array}{l}\text { condition } \times \text { test } \\
\text { time point }\end{array}$ & -0.0745 & 0.0269 & -2.766 & $.00567^{* *}$ \\
\hline \multirow[t]{4}{*}{ Experiment 2} & Thought/Emotion & $\begin{array}{l}\sim \text { condition } \times \text { test time point }+ \text { (condition }+ \\
\text { test time point } \mid \text { participant })\end{array}$ & .397 & .108 & intercept & 0.216 & 0.129 & 1.677 & .0936 \\
\hline & & & & & condition & 0.249 & 0.0423 & 5.882 & $4.06 \mathrm{e}-09^{* * *}$ \\
\hline & & & & & test time point & -0.128 & 0.0484 & -2.634 & $.00843^{* *}$ \\
\hline & & & & & $\begin{array}{l}\text { condition } \times \text { test } \\
\text { time point }\end{array}$ & -0.0421 & 0.0285 & -1.481 & .139 \\
\hline
\end{tabular}

Legend: $\mathrm{R}^{2} \mathrm{C}=$ conditional coefficient of determination, $\mathrm{R}^{2} \mathrm{M}$ : marginal coefficient of determination, SE: standard error, ${ }^{* *} P<.01$, ${ }^{* *} P<.001$. Note: models specified here are multilevel generalized Poisson models to best account for the count-based nature of the number of details recollected. 
ENHANCING REAL-WORLD MEMORY

Table S3. Intraclass correlations for intercept-only models of Internal and External detail counts.

\begin{tabular}{|c|c|c|c|c|}
\hline Analysis & Internal Details & ICC & External Details & ICC \\
\hline \multirow[t]{8}{*}{ Experiment 1} & Internal Total & .422 & External Total & .660 \\
\hline & Internal Event & .374 & External Event & .365 \\
\hline & Time & .186 & General Semantic & .465 \\
\hline & Place & .185 & Autobiographical Fact & .499 \\
\hline & Perceptual & .307 & Self-Knowledge & .332 \\
\hline & Thought/Emotion & .207 & Repeated Event & .242 \\
\hline & & & Repeated Detail & .360 \\
\hline & & & Other & .395 \\
\hline \multirow[t]{8}{*}{ Experiment 2} & Internal Total & .697 & External Total & .832 \\
\hline & Event & .655 & External Event & .645 \\
\hline & Time & .155 & General Semantic & .578 \\
\hline & Place & .243 & Autobiographical Fact & .715 \\
\hline & Perceptual & .572 & Self-Knowledge & .456 \\
\hline & Thought/Emotion & .240 & Repeated Event & .624 \\
\hline & & & Repeated Detail & .362 \\
\hline & & & Other & .298 \\
\hline
\end{tabular}




\subsection{Autobiographical Memory Test External Details by Subtype}

To provide a comprehensive picture of our data, we next examined whether HippoCamera replay differentially affected seven subtypes of External details (Fig. S3, Table S3-4).

External Event: There was no main effect of condition (Replayed vs. Baseline) in Experiment $1(b=-0.0758, \mathrm{SE}=0.0418, z=-1.816, p=.0694)$ or Experiment $2(b=$ $0.103, \mathrm{SE}=0.0643, z=-1.600, p=.110$ ). There was a significant main effect of test time point (Early vs. Delayed) in Experiment $1(b=-0.264, \mathrm{SE}=0.0753, z=-3.514, p<.001)$ and Experiment $2(b=-0.246, \mathrm{SE}=0.0698, z=-3.520, p<.001)$. In both experiments, participants provided more External Event details at Early than Delayed testing. The interaction between condition and test time point was not significant in Experiment $1(b=$ $-0.0252, \mathrm{SE}=0.0417, z=-0.604, p=.546)$ but was in Experiment $2(b=0.200, \mathrm{SE}=$ 0.0951, $z=2.106, p=.0352$ ). The interaction in Experiment 2 was driven by more External Event details at Early than Delayed for the Baseline condition $(b=-0.892, \mathrm{SE}=$ $0.254, z=-3.516, p<.001)$ but no difference across test time periods in the Replayed condition $(b=-0.0906, \mathrm{SE}=0.217, z=-0.418, p=.676)$.

General Semantics: The main effect of condition (Replayed vs. Baseline) was not significant in either Experiment $1(b=0.0293, \mathrm{SE}=0.0330, z=0.888, p=.374)$ or Experiment $2(b=0.0133, \mathrm{SE}=0.0536, z=0.248, p=.804)$. The main effect of test time point (Early vs. Delayed) was also not significant in Experiment $1(b=-0.0603$, SE $=$ 0.0866, $z=-0.696, p=.487)$ or Experiment $2(b=-0.0898, \mathrm{SE}=0.0496, z=-1.812, p=$ .070). There was a significant interaction between condition and test time point in Experiment $1(b=0.0868, \mathrm{SE}=0.0330, z=2.630, p=.00854)$ but not in Experiment 2 $(b=0.0339, \mathrm{SE}=0.0238, z=1.422, p=.155)$. For Experiment 1 , General Semantic details did not differ across Baseline and Replayed trials at Early testing $(b=-0.115$, SE $=0.0858, z=-1.340, p=.180$ ), whereas more details were recalled for Replayed than Baseline at Delayed testing $(b=0.232, \mathrm{SE}=0.100, z=2.314, p=.0207)$.

Autobiographical Facts: The main effect of condition (Replayed vs. Baseline) was not significant in either Experiment $1(b=-0.0287, \mathrm{SE}=0.0364, z=-0.786, p=.432)$ or Experiment $2(b=0.0203, \mathrm{SE}=0.0453, z=0.449, p=.654)$. There was a significant main 
effect of test time point (Early vs. Delayed) in both experiments (Experiment 1: $b=-0.135$, $\mathrm{SE}=0.0412, z=-3.265, p=.00109$; Experiment 2: $b=-0.210, \mathrm{SE}=0.0503, z=-4.177$, $p<.001)$, such that participants recalled more Autobiographical Fact details during Early as compared to Delayed testing. There were no significant interactions between condition and test time point in either experiment (Experiment 1: $b=0.00830, \mathrm{SE}=0.0223, z=$ $0.372, p=.710$; Experiment 2: $b=0.0467, \mathrm{SE}=0.0475, z=0.985, p=.325$ ).

Self-Knowledge: The main effect of condition was not significant in either Experiment 1 $(b=-0.0508, \mathrm{SE}=0.0417, z=-1.218, p=.223)$ or Experiment $2(b=0.00719, \mathrm{SE}=$ $0.0700, z=0.103, p=.918$ ). There was a significant main effect of test time point (Early vs. Delayed) in both experiments (Experiment 1: $b=-0.252$, SE $=0.0707, z=-3.559, p<$ .001 ; Experiment 2: $b=-0.118, \mathrm{SE}=0.0322, z=-3.652, p<.001$ ), such that participants recalled more Self-Knowledge details during Early as compared to Delayed testing. There were no significant interactions between condition and test time point in either experiment (Experiment 1: $b=-0.0168, \mathrm{SE}=0.0417, z=-0.402, p=.688$; Experiment 2: $b=0.0238$, $\mathrm{SE}=0.0322, z=0.739, p=.460)$.

Repeated Events: We found a significant main effect of condition (Replayed vs. Baseline) in Experiment $1(b=-0.323, \mathrm{SE}=0.122, z=-2.639, p=.00831)$ and Experiment $2(b=-$ $0.168, \mathrm{SE}=0.0657, z=-2.561, p=.0104)$. Across experiments, participants recalled more Repeated Event details for Baseline events compared to Replayed events. The main effect of test time point was not significant in either experiment (Experiment 1: $b=$ $0.182, \mathrm{SE}=0.112, z=-1.626, p=.104$; Experiment $2: b=-0.0725, \mathrm{SE}=0.0940, z=-$ $0.771, p=.441)$. The interaction between condition and test time point was not significant in either experiment (Experiment 1: $b=0.0131$, SE $=0.0762, z=0.172, p=.864$; Experiment 2: $b=0.0629, \mathrm{SE}=0.0617, z=1.019, p=.308$ ).

Repeated Details: We found a significant main effect of condition (Replayed vs. Baseline) in Experiment $1(b=0.0807, \mathrm{SE}=0.0321, z=2.518, p=.0118)$, with participants providing more Repeated details for Replayed events as compared to Baseline events. The effect of condition was not significant in Experiment $2(b=0.0431, \mathrm{SE}=0.0329, z=$ $1.310, p=.190)$. The effect of test time point was not significant in Experiment $1(b=-$ 0.132 , SE $=0.0738, z=-1.785, p=.0743)$ but was in Experiment $2(b=-0.210, \mathrm{SE}=$ 
0.0679, $z=-3.097, p=.00196)$. In Experiment 2, participants repeated more details at Early testing than they did at Delayed testing. The interaction between condition and test time point was not significant in either experiment (Experiment 1: $b=0.0171, \mathrm{SE}=0.0321$, $z=0.533, p=.594$; Experiment $2: b=0.0206, \mathrm{SE}=0.0272, z=0.760, p=.447$ ).

Other Details: We found a significant main effect of condition (Replayed vs. Baseline) in Experiment $1(b=-0.0567, \mathrm{SE}=0.0239, z=-2.370, p=.0178)$, which reflected more Other details for Baseline as compared to Replayed events. The effect of condition was not significant in Experiment $2(b=-0.0146, \mathrm{SE}=0.0434, z=-0.337, p=.736)$. The effect of test time point was not significant in either experiment (Experiment 1: $b=0.103, \mathrm{SE}=$ $0.0542, z=1.894, p=.0582$; Experiment 2: $b=0.00735$, SE $=0.0569, z=0.129, p=$ .897). There were also no significant interactions between condition and test time point (Experiment 1: $b=0.0243, \mathrm{SE}=0.0239, z=1.018, p=.309$; Experiment 2: $b=-0.0160$, $\mathrm{SE}=0.0224, z=-0.713, p=.476)$. 


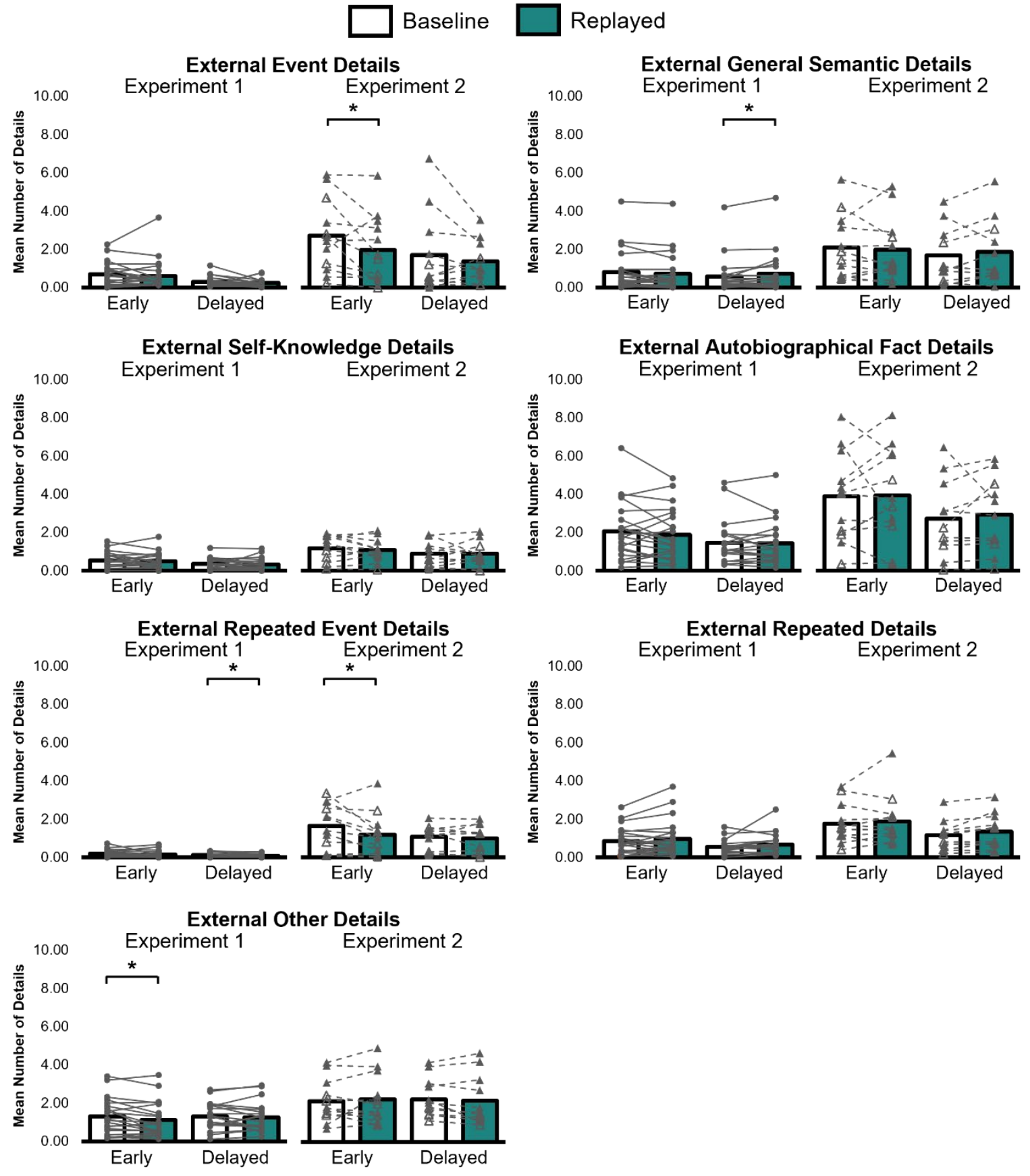

Fig. S3. Behavioral Results - External Details by Subtype. Mean number of External details by subtype for Experiment 1 and Experiment 2. Early testing corresponds to behavioral performance measured during (Experiment 1) and shortly after (Experiment 2) HippoCamera use. Delayed testing corresponds to behavioral performance after a 3-month delay, during which time participants did not have access to their memory cues. Open triangles denote Experiment 2 participants who failed the Montreal Cognitive Assessment. ${ }^{*}<.05$. 


\section{ENHANCING REAL-WORLD MEMORY}

Table S4. Fit statistics and fixed-effects parameters for best fitting models for External detail counts by subtype.

\begin{tabular}{|c|c|c|c|c|c|c|c|c|c|}
\hline Experiment & Detail Type & Model Formula & $\mathbf{R}^{2}{ }_{\mathrm{c}}$ & $\mathbf{R}^{2} \mathrm{M}$ & Fixed Effect & Estimate (b) & SE & $\mathbf{z}$ & $P$ \\
\hline \multirow[t]{4}{*}{ Experiment 1} & External Event & $\begin{array}{l}\sim \text { condition } \times \text { test time point }+ \text { (test time } \\
\text { point | participant) }\end{array}$ & .313 & .032 & intercept & -1.116 & 0.174 & -6.405 & $1.50 \mathrm{e}-10^{* * *}$ \\
\hline & & & & & condition & -0.0758 & 0.0418 & -1.816 & .0694 \\
\hline & & & & & test time point & -0.264 & 0.0753 & -3.514 & $.000442^{* * *}$ \\
\hline & & & & & $\begin{array}{l}\text { condition } \times \text { test time } \\
\text { point }\end{array}$ & -0.0252 & 0.0417 & -0.604 & .546 \\
\hline \multirow[t]{4}{*}{ Experiment 1} & $\begin{array}{l}\text { General } \\
\text { Semantic }\end{array}$ & $\begin{array}{l}\sim \text { condition } \times \text { test time point }+ \text { (test time } \\
\text { point | participant) }\end{array}$ & .483 & .005 & intercept & -0.961 & 0.230 & -4.172 & $3.02 \mathrm{e}-05^{* * *}$ \\
\hline & & & & & condition & 0.0293 & 0.0330 & 0.888 & .374 \\
\hline & & & & & test time point & -0.0603 & 0.0866 & -0.696 & .487 \\
\hline & & & & & $\begin{array}{l}\text { condition } \times \text { test time } \\
\text { point }\end{array}$ & 0.0868 & 0.0330 & 2.630 & .00854 ** \\
\hline \multirow[t]{4}{*}{ Experiment 1} & $\begin{array}{l}\text { Autobiographical } \\
\text { Fact }\end{array}$ & $\begin{array}{l}\sim \text { condition } \times \text { test time point }+ \text { (condition } \\
+ \text { test time point } \mid \text { participant) }\end{array}$ & .502 & .017 & intercept & 0.311 & 0.155 & 2.007 & .0447 * \\
\hline & & & & & condition & -0.0287 & 0.0364 & -0.786 & .432 \\
\hline & & & & & test time point & -0.135 & 0.0412 & -3.265 & $.00109 * *$ \\
\hline & & & & & $\begin{array}{l}\text { condition } \times \text { test time } \\
\text { point }\end{array}$ & 0.00830 & 0.0223 & 0.372 & .710 \\
\hline \multirow[t]{4}{*}{ Experiment 1} & Self-Knowledge & $\begin{array}{l}\text { condition } \times \text { test time point }+ \text { (test time } \\
\text { point | Participant) }\end{array}$ & .339 & .028 & intercept & -1.144 & 0.188 & -6.084 & $1.17 \mathrm{e}-09^{* * *}$ \\
\hline & & & & & condition & -0.0508 & 0.0417 & -1.218 & .223 \\
\hline & & & & & test time point & -0.252 & 0.0707 & -3.559 & $.000373^{* * *}$ \\
\hline & & & & & $\begin{array}{l}\text { condition } \times \text { test time } \\
\text { point }\end{array}$ & -0.0168 & 0.0417 & -0.405 & .688 \\
\hline
\end{tabular}




\section{ENHANCING REAL-WORLD MEMORY}

\begin{tabular}{|c|c|c|c|c|c|c|c|c|c|}
\hline Experiment & Detail Type & Model Formula & $\mathbf{R}^{2}{ }_{\mathrm{C}}$ & $\mathbf{R}^{2}{ }_{M}$ & Fixed Effect & Estimate (b) & SE & $\mathbf{z}$ & $P$ \\
\hline \multirow[t]{4}{*}{ Experiment 1} & Repeated Event & $\begin{array}{l}\sim \text { condition } \times \text { test time point }+ \text { (condition } \\
+ \text { test time point } \mid \text { participant })\end{array}$ & .289 & .038 & intercept & -2.405 & 0.225 & $-\overline{10.695}$ & $<2 \mathrm{e}-16^{* * *}$ \\
\hline & & & & & condition & -0.323 & 0.122 & -2.639 & .00831 ** \\
\hline & & & & & test time point & -0.182 & 0.112 & -1.626 & .104 \\
\hline & & & & & $\begin{array}{l}\text { condition } \times \text { test time } \\
\text { point }\end{array}$ & 0.0131 & 0.0762 & 0.172 & .864 \\
\hline \multirow[t]{4}{*}{ Experiment 1} & Repeated Detail & $\begin{array}{l}\sim \text { condition } \times \text { test time point }+ \text { (test time } \\
\text { point | participant) }\end{array}$ & .364 & .014 & intercept & -0.573 & 0.157 & -3.640 & $.000273^{* * *}$ \\
\hline & & & & & condition & 0.0807 & 0.0321 & 2.518 & $.0118^{*}$ \\
\hline & & & & & test time point & -0.132 & 0.0738 & -1.785 & .0743 \\
\hline & & & & & $\begin{array}{l}\text { condition } \times \text { test time } \\
\text { point }\end{array}$ & 0.0171 & 0.0321 & 0.533 & .594 \\
\hline \multirow[t]{4}{*}{ Experiment 1} & Other & $\begin{array}{l}\sim \text { condition } \times \text { test time point }+ \text { (test time } \\
\text { point | participant) }\end{array}$ & .440 & .013 & intercept & 0.0198 & 0.146 & 0.136 & .892 \\
\hline & & & & & condition & -0.0567 & 0.0239 & -2.370 & .0179 * \\
\hline & & & & & test time point & 0.103 & 0.0542 & 1.894 & .0582 \\
\hline & & & & & $\begin{array}{l}\text { condition } \times \text { test time } \\
\text { point }\end{array}$ & 0.0243 & 0.0239 & 1.018 & .309 \\
\hline \multirow[t]{4}{*}{ Experiment 2} & External Event & $\begin{array}{l}\sim \text { condition } \times \text { test time point }+ \text { (condition } \\
\times \text { test time point } \mid \text { participant })\end{array}$ & .703 & .056 & intercept & 0.142 & 0.309 & 0.459 & .646 \\
\hline & & & & & condition & -0.103 & 0.0643 & -1.600 & .110 \\
\hline & & & & & test time point & -0.246 & 0.0698 & -3.520 & $.000431^{* * *}$ \\
\hline & & & & & $\begin{array}{l}\text { condition } \times \text { test time } \\
\text { point }\end{array}$ & 0.200 & 0.0951 & 2.106 & .0352 * \\
\hline
\end{tabular}




\section{ENHANCING REAL-WORLD MEMORY}

\begin{tabular}{|c|c|c|c|c|c|c|c|c|c|}
\hline Experiment & Detail Type & Model Formula & $\mathbf{R}^{2} \mathrm{C}$ & $\mathbf{R}^{2}{ }_{M}$ & Fixed Effect & Estimate (b) & SE & $\mathbf{z}$ & $P$ \\
\hline \multirow[t]{4}{*}{ Experiment 2} & $\begin{array}{l}\text { General } \\
\text { semantic }\end{array}$ & $\begin{array}{l}\sim \text { condition } \times \text { test time point }+ \text { (condition } \\
+ \text { test time point | participant) }\end{array}$ & .586 & .007 & intercept & .0287 & 0.256 & 1.120 & .263 \\
\hline & & & & & condition & 0.0133 & 0.0536 & 0.248 & .804 \\
\hline & & & & & test time point & -0.0898 & 0.0496 & -1.812 & .070 \\
\hline & & & & & $\begin{array}{l}\text { condition } \times \text { test time } \\
\text { point }\end{array}$ & 0.0339 & 0.0238 & 1.422 & .155 \\
\hline \multirow[t]{4}{*}{ Experiment 2} & $\begin{array}{l}\text { Autobiographical } \\
\text { Fact }\end{array}$ & $\begin{array}{l}\sim \text { condition } \times \text { test time point }+ \text { (condition } \\
\times \text { test time point } \mid \text { participant) }\end{array}$ & .877 & .041 & intercept & 0.883 & 0.276 & 3.200 & $.00137^{* *}$ \\
\hline & & & & & condition & 0.0203 & 0.0453 & 0.449 & .654 \\
\hline & & & & & test time point & -0.210 & 0.0503 & -4.177 & $2.95 \mathrm{e}-05^{* * *}$ \\
\hline & & & & & $\begin{array}{l}\text { condition } \times \text { test time } \\
\text { point }\end{array}$ & 0.0467 & 0.0475 & 0.985 & .325 \\
\hline \multirow[t]{4}{*}{ Experiment 2} & Self-Knowledge & $\begin{array}{l}\sim \text { condition } \times \text { test time point }+ \text { (condition I } \\
\text { participant) }\end{array}$ & .470 & .009 & intercept & -0.252 & 0.242 & -1.041 & .298 \\
\hline & & & & & condition & 0.00719 & 0.0700 & 0.103 & .918 \\
\hline & & & & & test time point & -0.118 & 0.0322 & -3.652 & .000260 *** \\
\hline & & & & & $\begin{array}{l}\text { condition } \times \text { test time } \\
\text { point }\end{array}$ & 0.0238 & 0.0322 & 0.739 & .460 \\
\hline \multirow[t]{4}{*}{ Experiment 2} & Repeated Event & $\begin{array}{l}\sim \text { condition } \times \text { test time point }+(\text { condition } \\
\times \text { test time point } \mid \text { participant })\end{array}$ & .589 & .014 & intercept & -0.305 & 0.346 & -0.883 & .377 \\
\hline & & & & & condition & -0.168 & 0.0657 & -2.561 & .01404 * \\
\hline & & & & & test time point & -0.0725 & 0.0940 & -0.771 & .441 \\
\hline & & & & & $\begin{array}{l}\text { condition } \times \text { test time } \\
\text { point }\end{array}$ & 0.0629 & 0.0617 & 1.019 & .308 \\
\hline
\end{tabular}




\section{ENHANCING REAL-WORLD MEMORY}

\begin{tabular}{|c|c|c|c|c|c|c|c|c|c|}
\hline Experiment & Detail Type & Model Formula & $\mathbf{R}_{\mathrm{C}}^{2}$ & $\mathbf{R}^{2}{ }_{M}$ & Fixed Effect & Estimate (b) & SE & $\mathbf{z}$ & $P$ \\
\hline \multirow[t]{4}{*}{ Experiment 2} & Repeated Detail & $\begin{array}{l}\sim \text { condition } \times \text { test time point }+ \text { (condition } \\
+ \text { test time point | participant) }\end{array}$ & .440 & .048 & intercept & 0.230 & 0.171 & 1.347 & .178 \\
\hline & & & & & condition & 0.0431 & 0.0329 & 1.310 & .190 \\
\hline & & & & & test time point & -0.210 & 0.0679 & -3.097 & .00196 ** \\
\hline & & & & & $\begin{array}{l}\text { Replay condition } \times \\
\text { test time point }\end{array}$ & 0.0206 & 0.0272 & 0.760 & .447 \\
\hline \multirow[t]{4}{*}{ Experiment 2} & Other & $\begin{array}{l}\sim \text { condition } \times \text { test time point }+ \text { (condition } \\
+ \text { test time point | participant) }\end{array}$ & .356 & .001 & intercept & 0.661 & 0.125 & 5.289 & $1.23 e-07^{* * *}$ \\
\hline & & & & & condition & -0.0146 & 0.0434 & -0.337 & .736 \\
\hline & & & & & test time point & 0.00735 & 0.0569 & 0.129 & .897 \\
\hline & & & & & $\begin{array}{l}\text { condition } \times \text { test time } \\
\text { point }\end{array}$ & -0.0160 & 0.0224 & -0.713 & .476 \\
\hline
\end{tabular}

Legend: $\mathrm{R}_{\mathrm{C}}=$ conditional coefficient of determination, $\mathrm{R}^{2} \mathrm{M}$ : marginal coefficient of determination, SE: standard error, ${ }^{* *} P<.01,{ }^{* \star *} P<.001$. Note: models specified here are multilevel generalized Poisson models to best account for the count-based nature of the number of details recollected. 


\subsection{Autobiographical Memory Test Recall Density}

In addition to analyzing total detail counts, we also considered detail density, which controls for differences in verbal output across participants $(10,11)$. Internal and External density scores were derived for each trial by dividing respective detail counts by total word count. Using this approach produced results that converge with those obtained using detail counts as a dependent measure (Table S5).

For Internal density scores in Experiment 1 , we found a main effect of condition ( $b$ $=0.00575, \mathrm{SE}=0.000941, t(20)=6.111, p<.001)$ and test time point $(b=-0.00494$, SE $=0.000804, t(21)=-6.149, p<.001)$. Participants had higher Internal detail density scores both for Replayed events compared to Baseline events, and at Early testing as compared to Delayed testing. Additionally, there was a significant interaction between condition and test time point $(b=-0.00140, \mathrm{SE}=0.000575, t(1377)=-2.440, p=.0148)$. Participants had a significantly larger boost in their Internal detail density scores for Replayed events relative to Baseline events at Early testing $(b=0.0143$, SE $=0.00216, t(36)=6.611, p<$ $.001)$ than they did at Delayed testing $(b=0.00869$, SE $=0.00225, t(40)=3.872, p<$ $.001)$. In Experiment 2, we found a significant main effect of condition ( $b=0.00410$, SE $=$ $0.000516, t(929)=7.950, p<.001)$. As in Experiment 1, participants had higher Internal detail density scores for Replayed events compared to Baseline events. However, we found neither a significant main effect of test time point $(b=-0.000127$, SE $=0.000516$, $t(929)=-0.246, p=.806)$, nor a significant interaction between condition and test time point significant $(b=-0.000981, \mathrm{SE}=0.000516, t(929)=-1.901, p=.0576)$.

For External density scores in Experiment 1, we found a significant main effect of both condition $(b=-0.00265$, SE $=0.000521, t(1382)=-5.084, p<.001)$ and test time point $(b=0.00296, \mathrm{SE}=0.000778, t(19)=-3.807, p=.00111)$. Participants had higher External detail density scores for Baseline events compared to Replayed events, and at Early testing as compared to Delayed testing. The interaction between condition and test time point was not significant $(b=0.000592$, SE $=0.000520, t(1384)=1.137, p=.256)$. In Experiment 2, we also found a significant main effect of condition $(b=-0.00350$, SE = $0.000560, t(917)=-6.239, p<.001)$. Participants had higher External detail density scores for Baseline events compared to Replayed events. We did not find a significant 
main effect of test time point $(b=0.0000822, \mathrm{SE}=0.000715, t(10)=-0.115, p=.911)$ nor was the interaction between condition and test time point significant $(b=0.000271, \mathrm{SE}=$ $0.000560, t(917)=0.484, p=.629)$.

Overall, results obtained after converting cued recall responses to density scores converge with those obtained using raw detail counts. This outcome indicates that the effect of Replay on episodic recollection of everyday experiences is not driven by particularly loquacious individuals. This interpretation is also consistent with the fact that all participants showed a behavioral advantage in episodic recall for Replayed as compared to Baseline trials. 
Table S5. Fit statistics and fixed-effects parameters for best fitting models for density scores.

\begin{tabular}{|c|c|c|c|c|c|c|c|c|c|c|}
\hline Experiment & Density Score & Model Formula & $\mathbf{R}^{2} \mathrm{c}$ & $\mathbf{R}^{2}{ }_{M}$ & Fixed Effect & Estimate (b) & SE & df & $\mathbf{t}$ & $\mathbf{p}$ \\
\hline \multirow[t]{4}{*}{ Experiment 1} & Internal & $\begin{array}{l}\sim \text { condition } \times \text { test } \\
\text { time point }+ \\
\text { (condition }+ \text { test time } \\
\text { point I participant) }\end{array}$ & .381 & .081 & intercept & 0.0379 & 0.00309 & 21 & 12.283 & $\begin{array}{l}4.56 \mathrm{e}- \\
11^{* * *}\end{array}$ \\
\hline & & & & & condition & 0.00575 & 0.000941 & 20 & 6.111 & $\begin{array}{l}4.96 \mathrm{e}- \\
06^{* * *}\end{array}$ \\
\hline & & & & & $\begin{array}{l}\text { test time } \\
\text { point }\end{array}$ & -0.00494 & 0.000804 & 21 & -6.149 & $\begin{array}{l}4.09 \mathrm{e}- \\
06^{* * *}\end{array}$ \\
\hline & & & & & $\begin{array}{l}\text { condition } \times \\
\text { test time } \\
\text { point }\end{array}$ & -0.00140 & 0.000575 & 1377 & -2.441 & .0148 * \\
\hline \multirow[t]{4}{*}{ Experiment 1} & External & $\begin{array}{l}\sim \text { condition } \times \text { test } \\
\text { time point }+ \text { (test time } \\
\text { point I participant) }\end{array}$ & .219 & .033 & intercept & 0.0373 & 0.00206 & 21 & 18.114 & $\begin{array}{l}2.02 \mathrm{e}- \\
14^{* * *}\end{array}$ \\
\hline & & & & & condition & -0.00265 & 0.000521 & 1382 & -5.084 & $\begin{array}{l}4.20 \mathrm{e}- \\
07^{* * *}\end{array}$ \\
\hline & & & & & $\begin{array}{l}\text { test time } \\
\text { point }\end{array}$ & 0.00296 & 0.000778 & 19 & 3.807 & .00111 \\
\hline & & & & & $\begin{array}{l}\text { condition } \times \\
\text { test time } \\
\text { point }\end{array}$ & 0.000592 & 0.000520 & 1384 & 1.137 & .0256 \\
\hline
\end{tabular}




\begin{tabular}{|c|c|c|c|c|c|c|c|c|c|c|}
\hline Experiment & Density Score & Model Formula & $\mathbf{R}^{2} \mathrm{c}$ & $\mathbf{R}^{2} \mathrm{M}$ & Fixed Effect & Estimate (b) & SE & df & $\mathbf{t}$ & p \\
\hline \multirow[t]{4}{*}{ Experiment 2} & Internal & $\begin{array}{l}\sim \text { condition } \times \text { test } \\
\text { time point }+(1 \mid \\
\text { participant })\end{array}$ & .353 & .046 & intercept & 0.0256 & 0.00319 & 11 & 8.008 & $\begin{array}{l}6.49 \mathrm{e}- \\
06^{* * *}\end{array}$ \\
\hline & & & & & condition & 0.00410 & 0.000516 & 929 & 7.950 & $\begin{array}{l}5.39 \mathrm{e}- \\
15^{* * *}\end{array}$ \\
\hline & & & & & $\begin{array}{l}\text { test time } \\
\text { point }\end{array}$ & -0.000127 & 0.000516 & 929 & -0.246 & .806 \\
\hline & & & & & $\begin{array}{l}\text { condition } \times \\
\text { test time } \\
\text { point }\end{array}$ & -0.000981 & 0.000516 & 929 & -1.901 & .0576 \\
\hline \multirow[t]{4}{*}{ Experiment 2} & External & $\begin{array}{l}\sim \text { condition } \times \text { test } \\
\text { time point }+ \text { (test time } \\
\text { point | participant) }\end{array}$ & .276 & .030 & intercept & 0.0349 & 0.00292 & 11 & 11.944 & $\begin{array}{l}1.21 \mathrm{e}- \\
07^{* * *}\end{array}$ \\
\hline & & & & & condition & -0.00350 & 0.000560 & 917 & -6.239 & $\begin{array}{l}6.71 \mathrm{e}- \\
10^{* * *}\end{array}$ \\
\hline & & & & & $\begin{array}{l}\text { test time } \\
\text { point }\end{array}$ & $-8.217 e-05$ & 0.000715 & 10 & -0.115 & .911 \\
\hline & & & & & $\begin{array}{l}\text { condition } \times \\
\text { test time } \\
\text { point }\end{array}$ & 0.000271 & 0.000560 & 917 & 0.484 & .629 \\
\hline
\end{tabular}

Legend: $\mathrm{R}^{2} \mathrm{c}=$ conditional coefficient of determination, $\mathrm{R}^{2} \mathrm{M}=$ marginal coefficient of determination, $\mathrm{SE}=$ standard error, ${ }^{* *} p<.01,{ }^{* * *} p<.001$. 


\subsection{Sentiment Analyses}

We generated histograms of sentiment scores for all trials in order to provide a comprehensive picture that captures precisely how replay influence autobiographical sentiment (Fig. S4). Visual inspection suggests that the distribution of Replayed sentiment scores is shifted to be more positive than Baseline sentiment scores, with the most pronounced differences in the tails. Specifically, we find more highly positive outcomes and fewer negative outcomes for Replayed memories. In line with our inferential statistics, this shift is most apparent in Experiment 1 (2 weeks of HippoCamera use) and at Early testing. When considered together with autobiographical recall detail counts, these data suggest that beneficial effect of replay on episodic memory is more durable over time than is the effect of replay on sentiment.
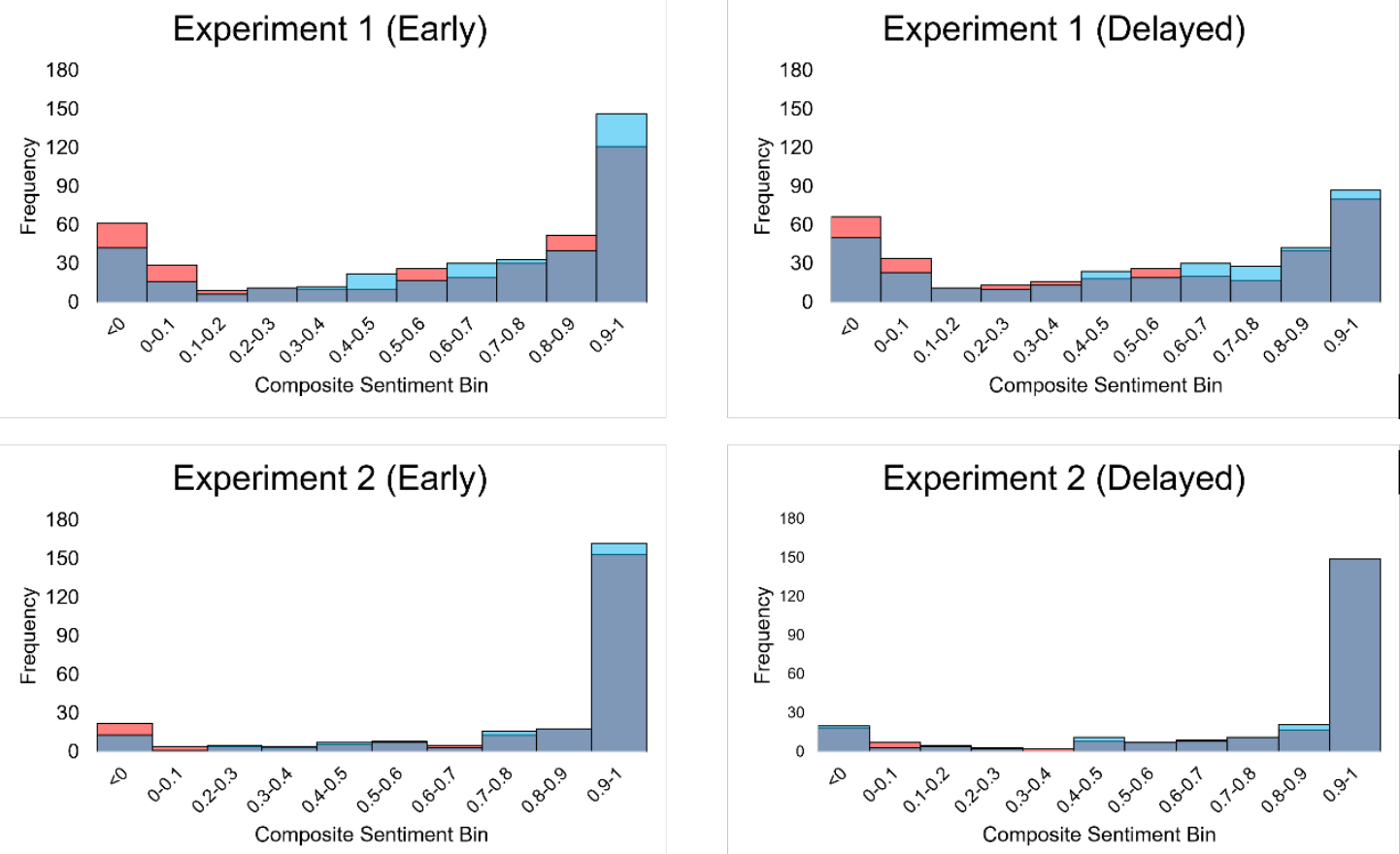

Fig. S4. Behavioral Results - Sentiment Histograms. Frequency distributions for composite sentiment scores for Early and Delayed testing in Experiments 1 and 2. Higher scores reflect more positive sentiment. 


\section{3. fMRI Methods}

\subsection{MRI and fMRI Acquisition}

MRI data were recorded on a 3T Siemens Magnetom Prisma system at the Toronto Neuroimaging facility using a 32-channel head coil. High-resolution anatomical images were acquired with a 3D-MPRAGE T1-weighted sequence with oblique axial slices covering the whole brain $\left(T R=2000 \mathrm{~ms}, \mathrm{TE}=2.4 \mathrm{~ms}\right.$, flip angle $=9^{\circ}$, voxel size $=1$ $\mathrm{mm}^{3}$, matrix size $=192 \times 256 \times 160$ ). Functional images were recorded using a gradient echo EPI sequence with 56 oblique axial slices oriented parallel to the hippocampus $\left(\mathrm{TR}=1000 \mathrm{~ms}, \mathrm{TE}=30 \mathrm{~ms}\right.$, flip angle $=45^{\circ}$, voxel size $=3.4 \times 3.4 \times 3.0 \mathrm{~mm}$, matrix size $=70 \times 70 \times 56$ ). The number of functional volumes acquired per run varied across participants $(M=298.25$, range $=265-327)$, reflecting the fact that some participants had more memories tested in the autobiographical interview than others.

\subsection{MRI and fMRI Preprocessing}

All neuroimaging data were preprocessed using FSL 6.00 (FMRIB software library, https://fsl.fmrib.ox.ac.uk/fsl/fslwiki). Images were skull-stripped using BET (12), and data were corrected for slice-acquisition time, high-pass temporally filtered using a 50second period cut-off, and motion corrected using MCFLIRT (13). Each participant's functional data were smoothed using a $5 \mathrm{~mm}$ FWHM kernel and registered to their highresolution anatomical image using FLIRT boundary-based registration. The resulting data were analyzed using first-level FEAT (14). Parameter estimates of BOLD response amplitude were computed using FILM, with a general linear model that included temporal autocorrelation correction and six motion parameters as nuisance covariates. Each task component of each trial (Watch Cue, Mentally Relive, and Episodic Probe) was modeled with a boxcar function corresponding to the event onset and then convolved with a double-gamma hemodynamic response function. Regressors were orthogonalized with respect to the preceding regressor using FSLs 'orthogonalise' function. Separate t-statistic images were created for each task component on each trial, which were then normalized to MNI space. Memory-specific t-images were then averaged across repetitions, resulting in a single t-image for each memory. These data were used for the purpose of our pattern-based similarity analyses. 


\subsection{ROI Definition}

All fMRI analyses were completed using data that were normalized to MNI space. Accordingly, one hippocampal ROI was created in each hemisphere using an MNI template. For the purpose of our first exploratory analysis, we used the uncal apex as an anatomical marker to create anterior hippocampus $\mathrm{ROI}$ that was distinct from a posterior hippocampus $\mathrm{ROI}$, bilaterally (15). We created seven additional ROls to for the purpose of a second set of exploratory analyses intended to quantify the neuroanatomical specificity of neuroimaging results obtained in the hippocampus. Six of these ROIs have figured prominently in $\mathrm{fMRI}$ and behavioral research on autobiographical memory in neurologically healthy populations and individuals with brain damage (vmPFC, ACC, PCC, angular gyrus, precuneus, and PHC; 16, 17). The seventh ROI, postcentral gyrus (i.e., primary somatosensory cortex) served as a control region that has not been systematically implicated in memory research. All exploratory analyses were performed using functional data combined across the left and right cerebral hemispheres. The vmPFC ROI was created using criteria established in previous research focused on memory consolidation (18). This ROI encompassed Brodmann's Area (BA) 14, BA 25, ventral parts of BA 24 and BA 32, the caudal part of BA 10, and the medial part of BA 11. ACC, PCC, angular gyrus, precuneus, and PHC ROIs were defined probabilistically using the Harvard-Oxford Cortical Atlas using 50\% as a voxel inclusion threshold.

\subsection{Representational Similarity Analysis}

Representational similarity analyses were performed using the CoSMoMVPA Matlab toolbox (http://www.cosmomvpa.org/) (19). Analyses focused on activity in the hippocampus, including exploratory analyses that examined the anterior and posterior extent separately, were performed using multivoxel patterns extracted from our ROls bilaterally.

\subsection{Linear Mixed Modeling with fMRI Data}

Data were analyzed using multilevel modeling in R 4.1.0 (R Core Team, 2021) using the same tools implemented in our behavioral analyses. We used 2-level multilevel linear models with individual trials nested within participants. Individual models were specified 
for the entire hippocampus, the anterior hippocampus, posterior hippocampus, and ventromedial prefrontal cortex (vmPFC). Multilevel modeling was appropriate given the degree of variance explained by individual participants for differentiation scores across all regions of interest, as assessed by their ICCs (Table S6). For all models, we would first always fit the model with the maximal random effects structure, according to Barr et al. (7). To investigate the effect of condition and task component, this entailed estimating fixed effects for condition (Baseline vs. Replayed), task (Episodic Probe vs. Mentally Relive vs. Watch Cue), and their interaction; a random intercept estimated for each participant; and a random slope estimated for each fixed effect. Both condition and task were effect coded (condition: Baseline $=-1$, Replayed $=1$; task: Episodic Probe $=1$, Mentally Relive $=0$, Watch Cue $=-1$; for second task component effect code: Episodic Probe $=0$, Mentally Relive $=1$, Watch Cue $=-1$ ). In the situation that the maximal model failed to converge due to overparameterization, we employed the backward-selection heuristic (8). To probe any significant interactions, simple effects tests were conducted with the Tukey adjustment for pairwise comparisons using the emmeans package (9)_ adjusted $\mathrm{P}$-values are reported for simple effects. The ImerTest package in $\mathrm{R}$ was used to obtain $P$-values corresponding to each fixed effect using likelihood ratio tests with the Satterthwaite approximation for degrees of freedom (20). The best fitting models described using Wilkinson notation, their corresponding model fit statistics, and ANOVA tables assessing the significance of fixed-effects parameters for the above analyses are summarized in Table S6. All models were estimated with an unstructured covariance matrix.

In addition, we performed an exploratory analysis to investigate whether differentiation scores differed across the long axis of the hippocampus. Specifically, we added an additional fixed effect for hippocampal ROI (Anterior vs. Posterior). Hippocampal ROls were effect coded (Anterior $=1$, Posterior $=-1$ ). Model specification was otherwise performed in the same fashion as described above.

\section{4. fMRI Results}

\subsection{Pattern Differentiation in the Anterior versus Posterior Hippocampus}


Our main set of fMRI pattern-based similarity analyses focused on the hippocampus in its entirety, which revealed greater differentiation (i.e., pattern dissimilarity) among memories in the Replayed condition than among memories in the Baseline condition (Fig. 4C). A substantial body of evidence suggests that the hippocampus is neither anatomically nor functional homogeneous along its anterior-posterior extent and that the functional distinction may be captured by differences between gist-based and detailbased memory representations $(21,22)$. Within this framework, the activity in the anterior hippocampus is thought to support gist-based memory, whereas the posterior hippocampus is thought to support detail-based memory. To investigate whether replayrelated increases in pattern differentiation differed between the anterior and posterior extent of the hippocampus, we performed an exploratory analysis that used the uncal apex as an anatomical marker separating the anterior from the posterior extent (23).

Differentiation scores are presented in Fig. S5. Model fits are presented in Table S6-7. A linear mixed model revealed a significant main effect of ROI, such that anterior hippocampus showed increased differentiation in activity patterns overall compared to posterior hippocampus $\left(b=6.143 \times 10^{-3}, \mathrm{SE}=2.855 \times 10^{-3}, t(4597)=2.152, P=.0351\right)$. However, we did not find evidence for any interactions involving ROI and any other predictor for differentiation in activity patterns (all $P$ 's $>.05$ ). We did find a significant main effect of condition (Replayed vs. Baseline: $b=2.587 \times 10^{-2}, \mathrm{SE}=2.855 \times 10^{-3}, t(4597)=$ $9.060, P<.001)$ and a significant interaction between condition and task $(F(2,4597)=$ 22.337, $P<.001)$. This was driven by increased differentiation in hippocampal activity patterns for Replayed compared to Baseline events during the Episodic Probe task $(b=$ 0.0886, SE $\left.=8.54 \times 10^{-3}, t(4597)=10.378, P<.001\right)$ and the Mentally Relive task $(b=$ $0.0582, \mathrm{SE}=0.0122, t(4597)=4.791, P<.001)$ tasks. The difference between Replayed and Baseline differentiation during the Watch Cue task was not significant $\left(b=8.37 \times 10^{-}\right.$ ${ }^{3}$, SE $\left.=8.54 \times 10^{-3}, t(4597)=0.980, P=.327\right)$. Lastly, we did not find evidence for a significant main effect of task $(F(2,13)=0.161, P=.853)$. 


\section{Anterior Hippocampus}

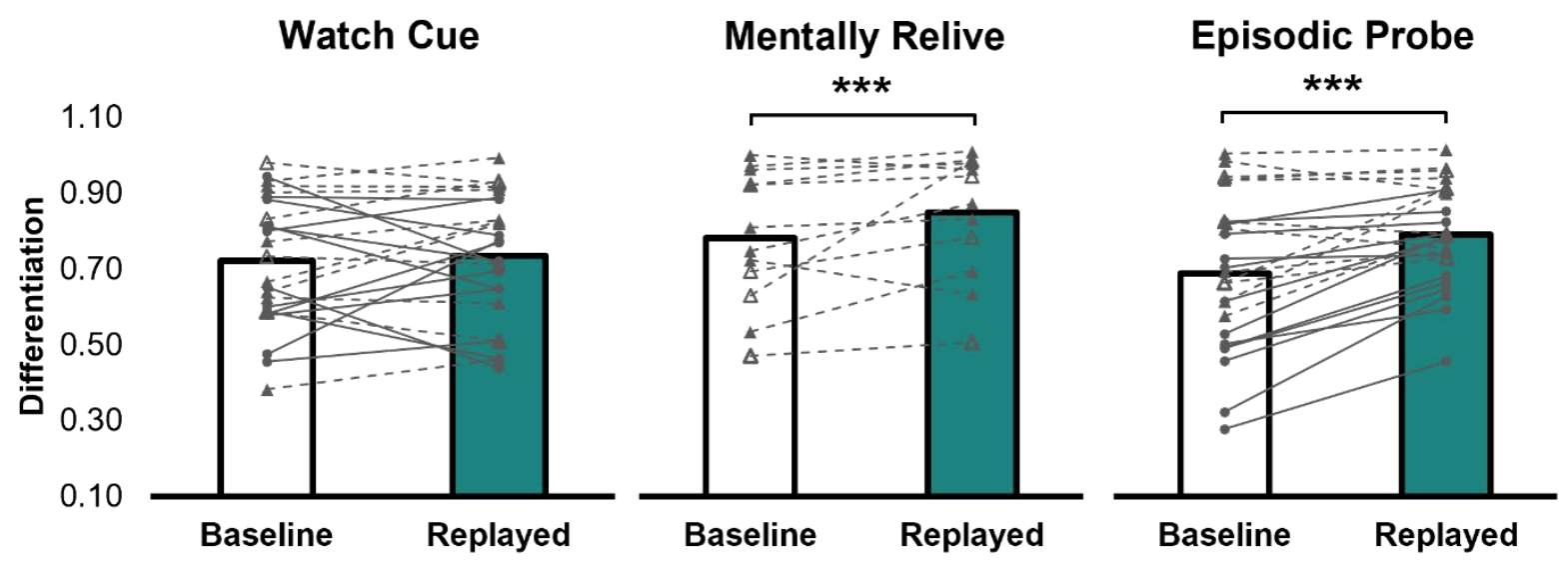

\section{Posterior Hippocampus}

Watch Cue

1.10

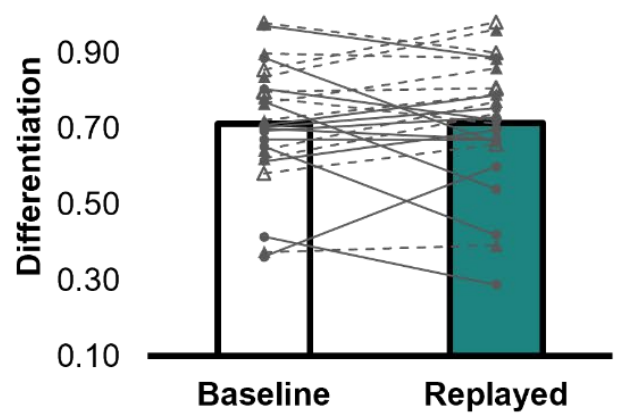

Mentally Relive

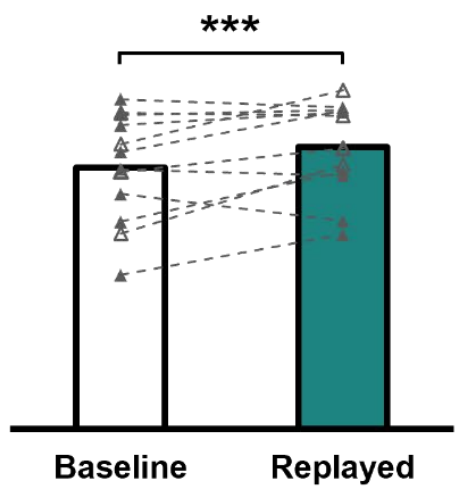

Episodic Probe

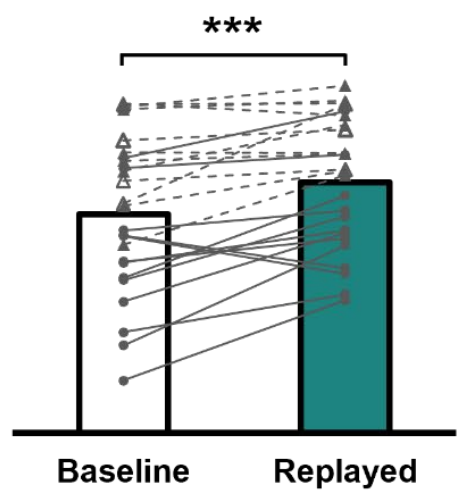

Fig. S5. Differentiation of Anterior and Posterior Hippocampus Activity. We quantified within condition measures of differentiation (1 - Pearson's $r$ ) using activity patterns obtained from the anterior and posterior hippocampus during each component of the fMRI task. Solid lines/circle markers depict data from Experiment 1 and dashed lines/triangle markers depict data from Experiment 2. Open triangles denote Experiment 2 participants who failed the Montreal Cognitive Assessment. ${ }^{* * *}=P<.001$.

\subsection{Pattern Differentiation Beyond the Hippocampus}

In addition to the well-established role of the hippocampus in the encoding and retrieval of memory for recent events, previous neuroimaging research has also revealed important contributions of vmPFC, ACC, PCC, angular gyrus, precuneus, and PHC (2428). We performed exploratory pattern analyses in these regions to characterize the neuroanatomical specificity of the replay-related increased differentiation we obtained 
when focusing on the hippocampus. We also included postcentral gyrus as a control ROI that has not figured prominently in memory research. See Fig. S6 for plots with data from individual participants and Table $\mathbf{S} 6$ for comprehensive fit statistics from a series of linear mixed models.

The main effect of condition (Replayed vs. Baseline) was not significant in any ROI after correcting for multiple comparisons (Bonferroni corrected threshold $=0.007$; vmPFC $P=0.0218$; Angular Gyrus $P=0.0684 ;$ Anterior Cingulate $P=0.1409$; Posterior Cingulate $P=0.0216$; Precuneus $P=0.0493$; Parahippocampal Cortex $P=0.2547$; Postcentral Gyrus $P=0.1772$ ). We note, however, that we did obtain marginal evidence for an effect of replay in vmPFC, Posterior Cingulate, and Precuneus (i.e., significant effects prior to correcting for multiple comparisons). The main effect of task (Watch Cue vs. Episodic Probe vs. Mentally Relive) was significant in Postcentral Gyrus ( $P=0.0040)$, an effect that was driven by greater overall differentiation in the Mentally Relive task. No other ROI showed a main effect of task (Bonferroni corrected threshold $=0.007$; vmPFC $P=0.2090$; Angular Gyrus $P=0.1093$; Anterior Cingulate $P=0.7812$; Posterior Cingulate $P=0.3016$; Precuneus $P=0.4209$; Parahippocampal Cortex $P=0.0775)$. Lastly, there was a significant interaction between condition and task in Parahippocampal Cortex $(P=$ 0.0006) and Precuneus $(P=0.0003)$, but not in any other ROI (Bonferroni corrected threshold $=0.007$; vmPFC $\mathrm{P}=0.0476$; Angular Gyrus $\mathrm{P}=0.1873$; Anterior Cingulate $\mathrm{P}=$ 0.0206; Posterior Cingulate $P=0.1497$; Postcentral Gyrus $P=0.0052$ ). The interactions in Parahippocampal Cortex and Precuneus were driven by increased differentiation for Replayed events compared to Baseline events in the Episodic Probe task; no differences were revealed between conditions in the Watch Cue or Mentally Relive tasks. 

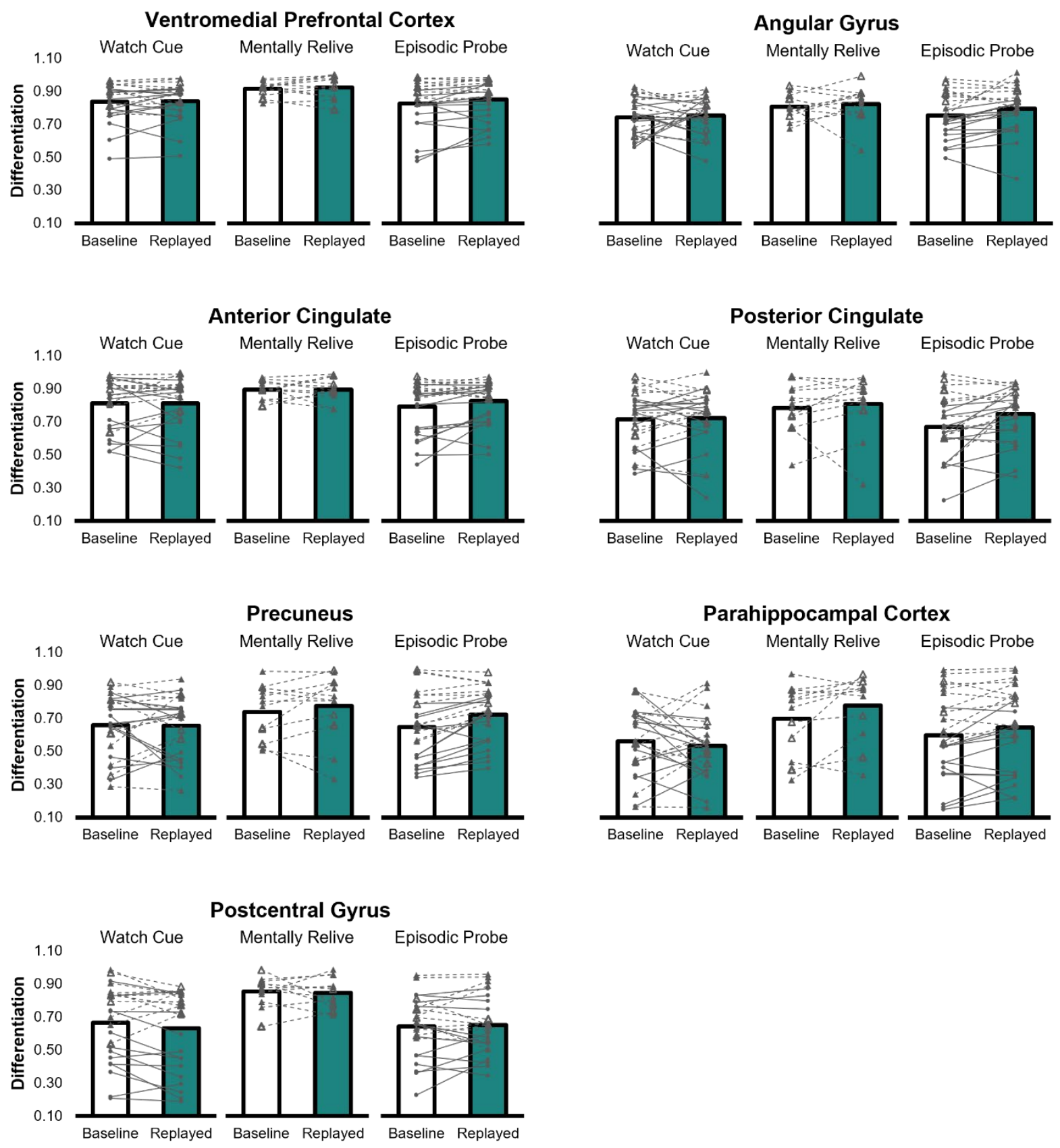

Fig. S6. Pattern differentiation beyond the hippocampus. We quantified differentiation (1 Pearson's r) using activity patterns obtained from seven exploratory ROls, including six that have figured prominently in research on autobiographical memory (ventromedial prefrontal cortex, angular gyrus, anterior cingulate, posterior cingulate, precuneus, and parahippocampal cortex) and one control region that has not been systematically linked to memory (postcentral gyrus). Solid lines/circle markers depict data from Experiment 1 and dashed lines/triangle markers depict data from Experiment 2. Open triangles denote Experiment 2 participants who failed the Montreal Cognitive Assessment. 
Table S6. Fit statistics and fixed-effects parameters for best fitting models of fMRI differentiation by region of interest

\begin{tabular}{|c|c|c|c|c|c|c|c|c|c|c|}
\hline Analysis (ROI) & Model Formula & $\mathbf{R}^{2} \mathrm{C}$ & $\mathbf{R}^{2} \mathbf{M}$ & Fixed Effect & sS & MS & $\mathbf{d f}_{\mathrm{N}}$ & $\mathbf{d f}_{\mathrm{D}}$ & $\mathbf{F}$ & $P$ \\
\hline \multirow[t]{3}{*}{ Hippocampus } & $\begin{array}{l}\sim \text { condition } \times \text { task }+(\text { condition }+ \\
\text { task | participant })\end{array}$ & .500 & .022 & condition & 0.0479 & 0.479 & 1 & 24 & 17.508 & $.000330^{* * *}$ \\
\hline & & & & task & 0.0838 & 0.0419 & 2 & 11 & 1.532 & .257 \\
\hline & & & & condition $\times$ task & 0.762 & 0.381 & 2 & 2186 & 13.933 & $9.71 \mathrm{e}-07^{* * *}$ \\
\hline \multirow[t]{7}{*}{$\begin{array}{l}\text { Anterior/Posterior } \\
\text { Hippocampus }\end{array}$} & $\begin{array}{l}\sim \text { condition } \times \text { task } \times \text { region of } \\
\text { Interest }+ \text { (task | participant) }\end{array}$ & .423 & .018 & condition & 2.800 & 2.800 & 1 & 4597 & 82.076 & $<2.2 \mathrm{e}-16^{* * *}$ \\
\hline & & & & task & 0.0110 & 0.00549 & 2 & 13 & 0.161 & .853 \\
\hline & & & & region of Interest & 0.158 & 0.158 & 1 & 4597 & 4.629 & $.0315^{*}$ \\
\hline & & & & condition $\times$ task & 1.524 & 0.762 & 2 & 4597 & 22.337 & $2.22 \mathrm{e}-10$ *** \\
\hline & & & & condition $\times$ region of Interest & 0.0305 & 0.0305 & 1 & 4597 & 0.863 & .345 \\
\hline & & & & $\begin{array}{l}\text { task } \times \\
\text { region of Interest }\end{array}$ & 0.0645 & 0.0322 & 2 & 4597 & 0.945 & .389 \\
\hline & & & & $\begin{array}{l}\text { condition } \times \text { task } \times \text { region of } \\
\text { Interest }\end{array}$ & 0.00402 & 0.00201 & 2 & 4597 & 0.0589 & .943 \\
\hline \multirow[t]{3}{*}{$\begin{array}{l}\text { ventromedial } \\
\text { Prefrontal Cortex }\end{array}$} & $\begin{array}{l}\sim \text { condition } \times \text { task }+(1 \mid \\
\text { participant })\end{array}$ & .473 & .004 & condition & 0.0762 & 0.0762 & 1 & 2303 & 5.272 & .0218 \\
\hline & & & & task & 0.0452 & 0.0226 & 2 & 2309 & 1.566 & .209 \\
\hline & & & & condition $\times$ task & 0.0881 & 0.0440 & 2 & 2303 & 3.048 & .0476 \\
\hline \multirow[t]{3}{*}{ Angular Gyrus } & $\begin{array}{l}\sim \text { condition } \times \text { task }+ \text { (condition }+ \\
\text { task | participant) }\end{array}$ & .294 & .010 & condition & .1061 & .1061 & 1 & 25.50 & 3.621 & .0684 \\
\hline & & & & task & .1508 & .0754 & 2 & 15.19 & 2.571 & .1093 \\
\hline & & & & condition $\times$ task & .0983 & .0492 & 2 & 2135.33 & 1.676 & .1873 \\
\hline
\end{tabular}




\begin{tabular}{|c|c|c|c|c|c|c|c|c|c|c|}
\hline Analysis (ROI) & Model Formula & $\mathbf{R}^{2} \mathrm{C}$ & $\mathbf{R}^{2} \mathrm{M}$ & Fixed Effect & SS & MS & $\mathbf{d f _ { N }}$ & $\mathbf{d f}_{\mathrm{D}}$ & $\mathbf{F}$ & $P$ \\
\hline \multirow[t]{3}{*}{ Anterior Cingulate } & $\begin{array}{l}\sim \text { condition } \times \text { task }+(1 \mid \\
\text { participant })\end{array}$ & .465 & .003 & condition & .0461 & .0461 & 1 & 2299.9 & 2.169 & .1409 \\
\hline & & & & task & .0105 & .0052 & 2 & 2305.4 & 0.247 & .7812 \\
\hline & & & & condition $\times$ task & .1653 & .0827 & 2 & 2299.9 & 3.89 & .0206 \\
\hline \multirow[t]{3}{*}{ Posterior Cingulate } & $\begin{array}{l}\sim \text { condition } \times \text { task }+ \text { (condition * } \\
\text { task | participant) }\end{array}$ & .480 & .017 & condition & .2086 & .2086 & 1 & 14.456 & 6.626 & .0216 \\
\hline & & & & task & .0800 & .0400 & 2 & 20.686 & 1.271 & .3016 \\
\hline & & & & condition $\times$ task & .1328 & .0664 & 2 & 18.411 & 2.109 & .1497 \\
\hline \multirow[t]{3}{*}{ Precuneus } & $\begin{array}{l}\sim \text { condition } \times \text { task }+ \text { (condition }+ \\
\text { task | participant) }\end{array}$ & .496 & .011 & condition & .1452 & .1452 & 1 & 25.90 & 4.255 & .0493 \\
\hline & & & & task & .0629 & .0315 & 2 & 13.69 & 0.922 & .4209 \\
\hline & & & & condition $\times$ task & .5665 & .2832 & 2 & 2199.07 & 8.302 & $.0003^{\dagger}$ \\
\hline \multirow[t]{3}{*}{ Parahipp. Cortex } & $\begin{array}{l}\sim \text { condition } \times \text { task }+ \text { (condition }+ \\
\text { task } \mid \text { participant })\end{array}$ & .547 & .017 & condition & .0599 & .0599 & 1 & 25.79 & 1.357 & .2547 \\
\hline & & & & task & .2723 & .1361 & 2 & 14.12 & 3.081 & .0775 \\
\hline & & & & condition $\times$ task & .6599 & .3299 & 2 & 2213.21 & 7.467 & $.0006^{\dagger}$ \\
\hline \multirow[t]{3}{*}{ Postcentral Gyrus } & $\begin{array}{l}\sim \text { condition } \times \text { task }+ \text { (condition }+ \\
\text { task | participant) }\end{array}$ & .582 & .013 & condition & .0569 & .0569 & 1 & 26.42 & 1.923 & .1772 \\
\hline & & & & task & .7069 & .3535 & 2 & 7.94 & 11.939 & .0040 \\
\hline & & & & condition $\times$ task & .3121 & .1560 & 2 & 2174.21 & 5.271 & .0052 \\
\hline
\end{tabular}

Legend: $R^{2} C=$ conditional coefficient of determination, $R^{2} \mathrm{M}$ : marginal coefficient of determination, $\mathrm{SS}$ : sum of squares, MS: mean squares, dfN: numerator degrees of freedom, dfD: denominator degrees of freedom, ${ }^{*} P<.05,{ }^{* \star} P<.01,{ }^{* * \star} P<.001,{ }^{\dagger} \mathrm{P}<.007$ (Bonferroni corrected significance threshold for exploratory analyses). 
ENHANCING REAL-WORLD MEMORY

Table S7. Intraclass correlations for intercept-only models of fMRI differentiation by region of interest

\begin{tabular}{ll} 
Analysis (ROI) & ICC \\
\hline Hippocampus & .391 \\
Anterior/Posterior Hippocampus & .349 \\
Ventromedial Prefrontal Cortex & .475 \\
Angular Gyrus & .187 \\
Anterior Cingulate & .464 \\
\hline Posterior Cingulate & .364 \\
\hline Precuneus & .376 \\
Parahippocampal Cortex & .369 \\
\hline Postcentral Gyrus & .524 \\
\hline
\end{tabular}




\subsection{Relationship Between Pattern Differentiation in the Hippocampus and Recall of External Details}

In a final set of exploratory analyses, we correlated differentiation scores from the hippocampus and number of External details recalled in our Early and Delayed behavioral assessments (Fig. S7). Whereas hippocampal differentiation was significantly correlated with number of Internal details recalled (Fig. 5 in main text), we found limited evidence for any such relationship with External details. A one-sample t-test against a mean of zero revealed that the group averaged correlation values were significantly greater than chance for the Mentally Relive task at Early testing $(t(11)=2.23, P<.05)$. No other correlations were significantly greater than chance (Watch Cue and Early testing $(t(24)=$ $0.286, P=.777)$, Watch Cue and Delayed testing $(t(22)=-0.866, P=.396)$, Mentally Relive and Delayed testing $(t(11)=-0.224, P=.827)$, Episodic Probe and Early testing $(t(24)=-0.469, P=.643)$, or Episodic Probe and Delayed testing $(t(22)=1.59, P=.124)$.

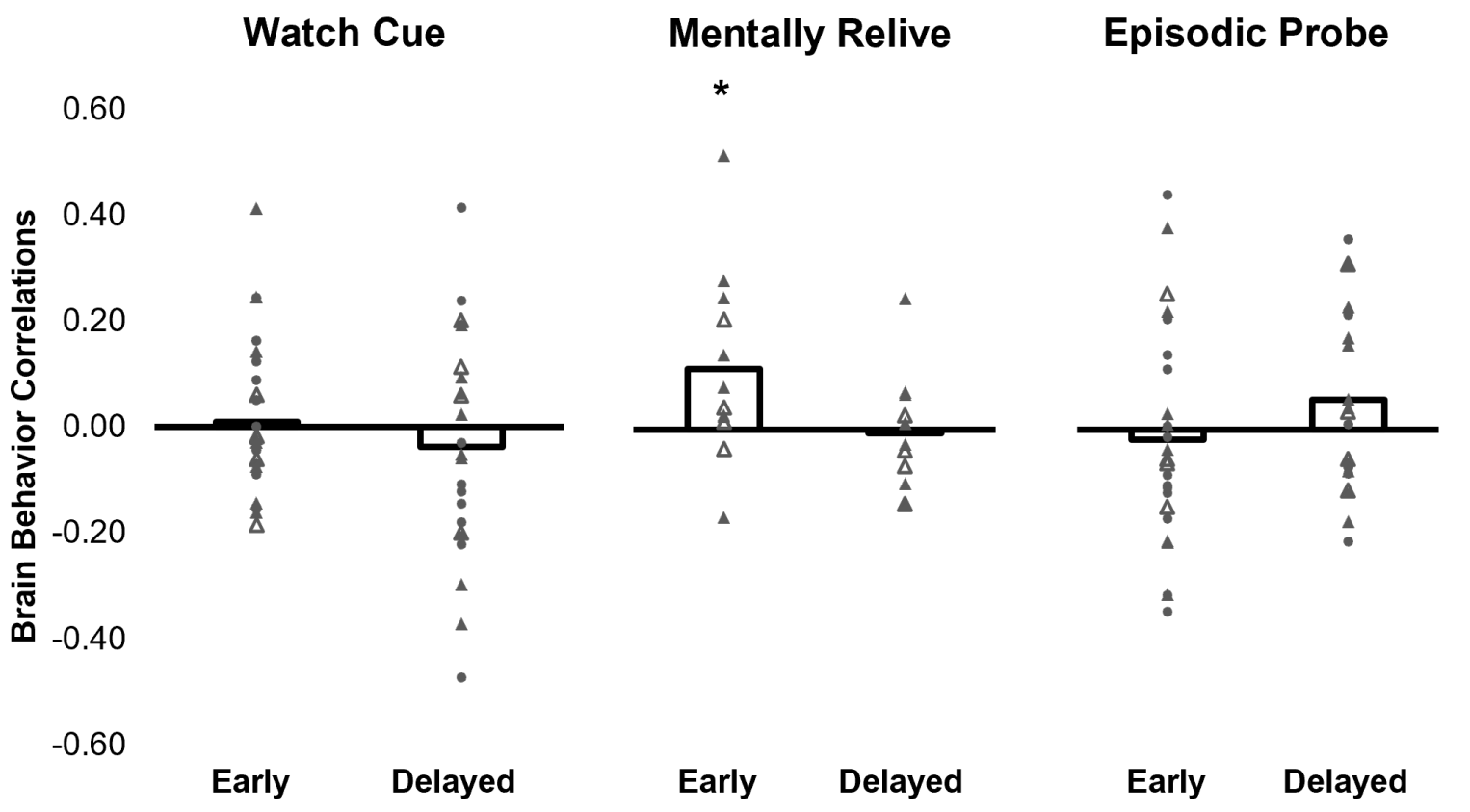

Fig. S7. Correlation Between Differentiation Scores in the Hippocampus and Retrieval of External Details. Pearson's correlation values obtained between hippocampal differentiation and overall number of External details recalled at Early testing and Delayed testing. Circles reflect participants from Experiment 1. Triangles reflect participants from Experiment 2. Open triangles denote Experiment 2 participants who failed the Montreal Cognitive Assessment. ${ }^{*}<.05$ 


\section{Single-Case Study in Hippocampal Amnesia}

Here we report behavioral and neuroimaging data that reveal the potential of

HippoCamera as a digital memory aid in older adults, including a subset of participants who are at risk for cognitive decline. An important outstanding question is whether this cognitive behavioral intervention will similarly benefit participants with frank memory disorders. To this end, we complement our primary findings with evidence from an individual who suffers from dense hippocampal amnesia.

\subsection{Participant}

We tested HippoCamera in a 28 year-old woman (LP) who developed temporal lobe encephalitis due to a viral infection. Review of a T1-weighted MR image revealed near complete ablation of posterior hippocampus bilaterally but some sparing of anterior hippocampus. Further abnormalities are evident in the amygdala and medial temporal lobe cortical areas (i.e., entorhinal cortex, perirhinal cortex, and parahippocampal cortex). She was left profoundly amnesic (e.g., Logical Memory Immediated $=4 / 25$, Delayed $=0 / 25$ ) and showed considerable evidence of general cognitive decline (e.g., $6 / 30$ on the Montreal Cognitive Assessment).
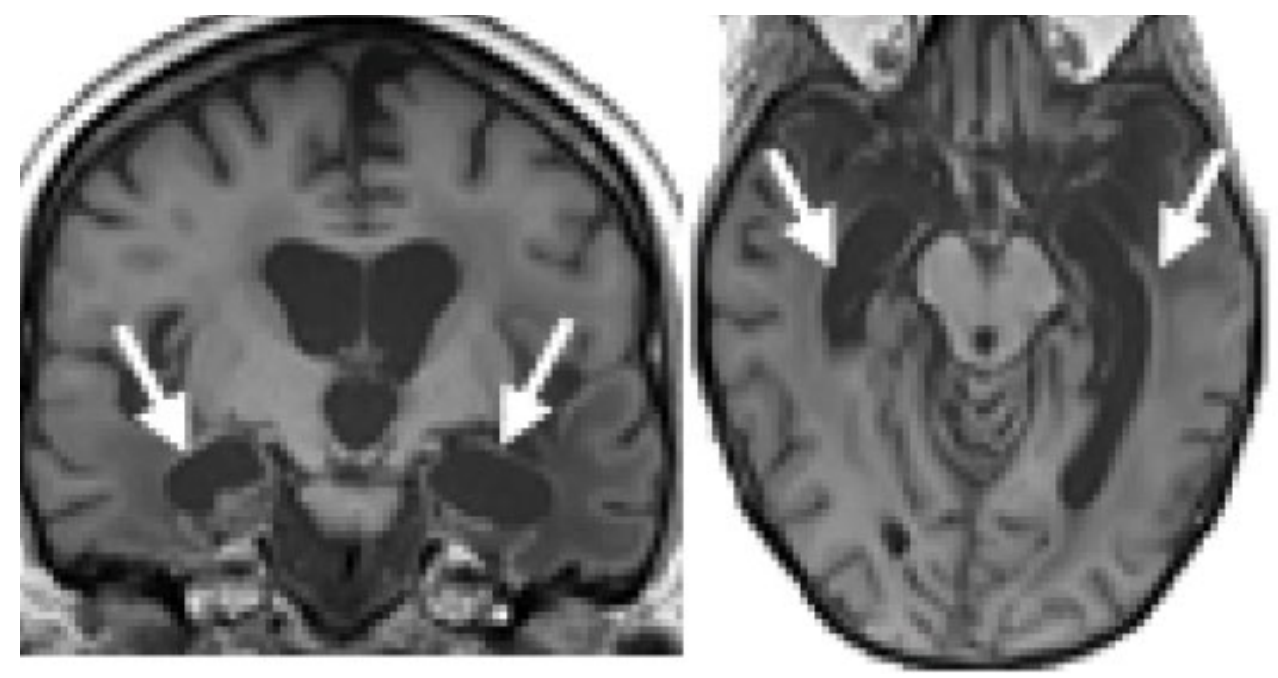

Fig. S8. T1-Weighted MR Image of L.P.'s Bilateral Medial Temporal Lobe Lesion. White arrows highlight extensive tissue loss in the hippocampus, amygdala, entorhinal cortex, perirhinal cortex, and parahippocampal cortex. 


\subsection{Experimental Design and Results}

At intake we noticed that LP diligently kept a diary with approximately 10 entries per day. Moreover, she would often review it to make conversation or reminder herself of an event. In addition to comparing memory for Replayed vs. Baseline events captured using HippoCamera, we also used her written notes as a diary control condition. LP recorded 49 events (2.2/day) and viewed 106 Replay sessions (4.8/day) over a 3-week period. HippoCamera memory cues were assigned to the Replayed and Baseline condition randomly in an interleaved manner (i.e., the same procedure used for the purpose of Experiment 1).

At test, we showed LP cues from either her diary ( $N=13$ trials), the Baseline HippoCamera condition ( $N=10$ trials), or the Replayed HippoCamera condition ( $N=12$ trials) and asked her to describe each event. She produced External details for all tested events (mean number per event: Diary $=2.92$; Baseline HippoCamera $=2.30$; Replayed HippoCamera $=3.08$ ), as is typical of patients with hippocampal damage. She recalled an equal number of Internal details for events in the Diary condition (mean = 0.54 ) and Baseline HippoCamera condition (mean $=0.50$ ). Critically, however, she produced nearly three times as many Internal details for events replayed with HippoCamera $($ mean $=1.50$ ) relative to either her Diary or the Baseline HippoCamera condition (Fig. S9). Notably, while she produced no episodic details for 3-week old events recorded with her diary, she produced some details for 3-week old events recorded with HippoCamera. Anecdotally, her mother recounted a "breakthrough moment" (her words) in which the patient described an important event recorded with HippoCamera - a kayak ride at her friend's cottage. Critically, this memory was produced spontaneously in conversation (i.e., without cues), suggesting that HippoCamera might engender flexible retrieval of events in a manner that journal entries cannot. Lastly, these findings suggest that HippoCamera may have therapeutic value for individuals with memory disorders. 


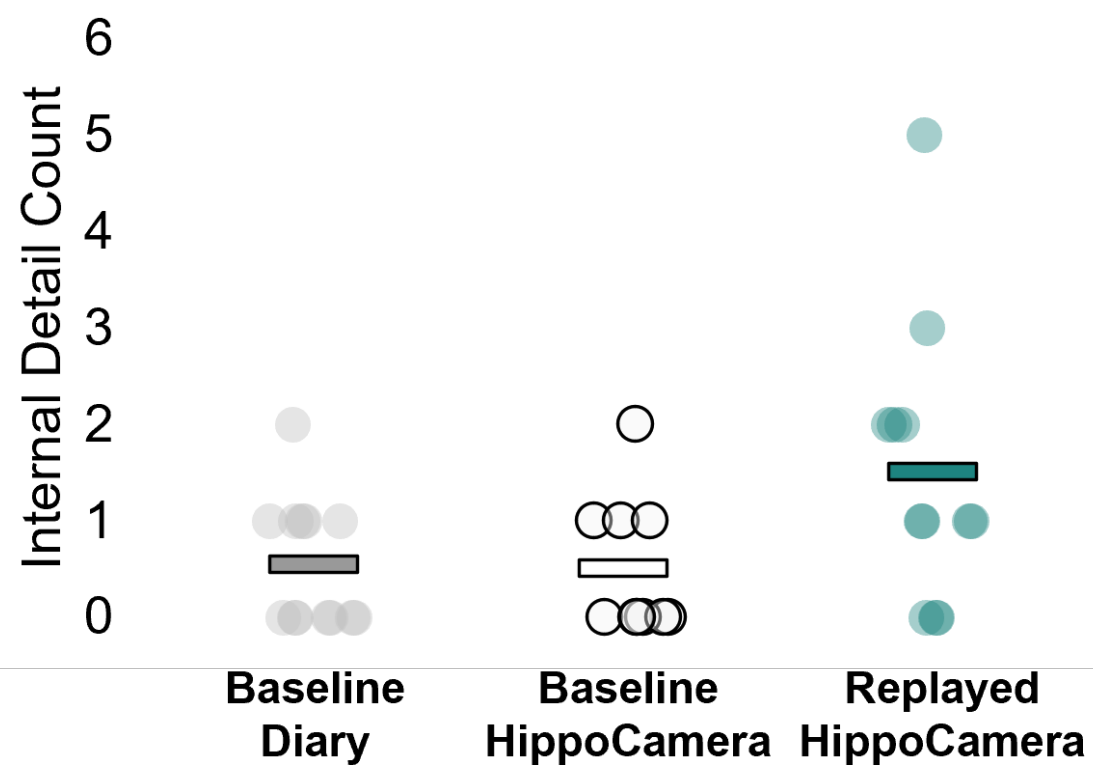

Fig. S9. HippoCamera Replay Improved Recall of Internal Details in LP. Bars reflect mean values for each condition. Individual markers reflect performance on each trial within a given condition.

\section{References}

1. Levine B, Svoboda E, Hay JF, Winocur G, Moscovitch M (2002) Aging and autobiographical memory: dissociating episodic from semantic retrieval. Psychol Aging 17:677-689.

2. Renoult L, Armson MJ, Diamond NB, Fan CL, Jeyakumar N, Levesque L, et al. (2020) Classification of general and personal semantic details in the Autobiographical Interview. Neuropsychologia, 144: 107501.

3. Bates D, Mächler M, Bolker B, Walker S (2015) Fitting linear mixed-effects models using Ime4. J Stat Softw, 67(1).

4. Lüdecke D, Ben-Shachar MS, Patil I, Waggoner P, Makowski D (2021) Performance: An R package for assessment comparison and testing of statistical models. Journal of Open Source Software, 6.

5. Nakagawa S, Johnson PC, Schielzeth $H(2017)$ The coefficient of determination R2 and intra-class correlation coefficient from generalized linear mixed-effect models revisited and expanded. J R Soc Interface 14(134):20170213. 
6. Bolker BM, Brooks ME, Clark CJ, Geange SW, Poulsen JR, Stevens MHH, White JSS (2009) Generalized linear mixed models: a practical guide for ecology and evolution. Trends Ecol Evol 24(3):127-135.

7. Barr DJ, Levy R, Scheepers C, Tily HJ (2013) Random effects structure for confirmatory hypothesis testing: Keep it maximal. J Mem Lang 68(3):255-278.

8. Matuschek H, Kliegl R, Vasishth S, Baayen H, Bates D (2017) Balancing type I error and power in linear mixed models. J Mem Lang 94:305-315.

9. Lenth RV (2021) Emmeans: Estimated marginal means, aka least-squares means. $\mathrm{R}$ package version 1.6.1.

10. Lockrow AW, Setton R, Spreng KAP, Sheldon S, Turner GR, Spreng RN (2021) Taking stock of the past: a psychometric evaluation of the Autobiographical Interview. bioRxiv https://doi.org/10.1101/2021.12.22.473803

11. Setton R, Sheldon S, Turner GR, Spreng RN (2022) Temporal pole volume is associated with episodic autobiographical memory in healthy older adults. Hippocampus 32(5):373-385.

12. Smith SM (2002) Fast robust automated brain extraction. Hum Brain Mapp 17(3):143-155.

13. Jenkinson M, Bannister P, Brady M, Smith S (2002). Improved optimization for the robust and accurate linear registration and motion correction of brain images. Neuroimage 17(2):825-841.

14. Jenkinson M, Beckmann CF, Behrens TE, Woolrich MW, Smith SM (2012) FSL. Neuroimage 62(2):782-790.

15. Poppenk J, Moscovitch M (2011) A hippocampal marker of recollection memory ability among healthy young adults: contributions of posterior and anterior segments. Neuron 72(6):931-937.

16. Svoboda E, McKinnon MC, Levine B (2006) The functional neuroanatomy of autobiographical memory: a meta-analysis. Neuropsychologia, 44(12):2189-2208.

17. Schacter DL, Addis DR, Buckner RL (2007) Remembering the past to imagine the future: the prospective brain. Nat Rev Neurosci 8(9):657-661. 
18. Bonnici HM et al (2012) Detecting representations of recent and remote autobiographical memories in vmPFC and hippocampus. J Neurosci 32(47):1698216991.

19. Oosterhof NN, Connolly AC, Haxby JV (2016). CoSMoMVPA: multi-modal multivariate pattern analysis of neuroimaging data in Matlab/GNU Octave. Front Neuroinform 10:27.

20. Kuznetsova A, Brockhoff PB, Christensen RH (2017) ImerTest package: Tests in linear mixed effects models. J Stat Softw 82(1):1-26.

21. Poppenk J, Evensmoen HR, Moscovitch M, Nadel L (2013) Long-axis specialization of the human hippocampus. Trends Cogn Sci 17(5):230-240.

22. Brunec IK et al (2018) Multiple scales of representation along the hippocampal anteroposterior axis in humans. Curr Biol 28(13):2129-2135.

23. Poppenk J, Moscovitch M (2011) A hippocampal marker of recollection memory ability among healthy young adults: contributions of posterior and anterior segments. Neuron 72(6):931-937.

24. Gilboa A (2004) Autobiographical and episodic memory-one and the same?: Evidence from prefrontal activation in neuroimaging studies. Neuropsychologia 42(10):1336-1349.

25. Cabeza R, St. Jacques $P$ (2007) Functional neuroimaging of autobiographical memory. Trends Cogn Sci 11(5):219-227.

26. Bonnici HM et al (2012) Detecting representations of recent and remote autobiographical memories in vmPFC and hippocampus. J Neurosci 32(47):1698216991.

27. Svoboda E, McKinnon MC, Levine B (2006) The functional neuroanatomy of autobiographical memory: a meta-analysis. Neuropsychologia, 44(12):2189-2208.

28. Schacter DL, Addis DR, Buckner RL (2007) Remembering the past to imagine the future: the prospective brain. Nat Rev Neurosci 8(9):657-661. 